\title{
Autoimmune T cell - B cell interaction in experimental autoimmune encephalomyelitis
}

\author{
Dissertation \\ for the award of the degree \\ "Doctor rerum naturalium" \\ of the Georg-August-Universität Göttingen \\ within the doctoral program "Molecular Biology of Cells" \\ of the Georg-August University School of Science (GAUSS)
}

submitted by

Anne-Christine Flach

from Remscheid

Göttingen 2014 
Members of the Thesis Committee:

- $\quad 1^{\text {st }}$ Reviewer: Prof. Dr. Jürgen Wienands, Department of Cellular and Molecular Immunology, University Medical School, Göttingen

- $2^{\text {nd }}$ Reviewer: Prof. Dr. Mikael Simons, Cellular Neuroscience, Max Planck Institute of Experimental Medicine and Department of Neurology, Göttingen

- Prof. Dr. Alexander Flügel, Department of Neuroimmunology, Institute for Multiple Sclerosis Research, University Medical School, Göttingen

Additional Members of the Examination Board:

- Prof. Dr. Holger Reichardt, Department of Cellular and Molecular Immunology, Institute for Experimental Immunology, University Medical School, Göttingen

- Prof. Dr. Uwe-Karsten Hanisch, Department of Neuropathology, University Medical School, Göttingen

- Prof. Dr. Lutz Walter, Department of Primate Genetics, German Primate Center, Göttingen 


\section{Declaration}

I hereby declare that I have written the $\mathrm{PhD}$ thesis entitled "Autoimmune $\mathrm{T}$ cell - B cell interaction in experimental autoimmune encephalomyelitis" on my own with no other sources and aids than quoted. The thesis has not been submitted elsewhere for any academic degree.

Anne-Christine Flach

Göttingen, May 2014 


\section{Table of contents}

Figure list

Table list

Abbreviations

Abstract

1. Introduction

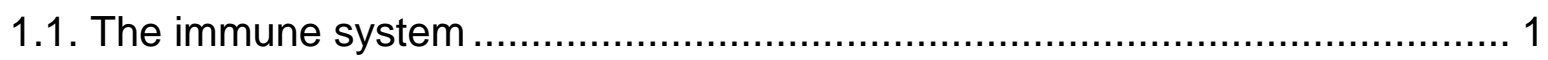

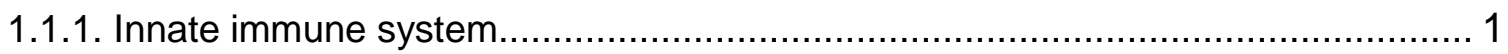

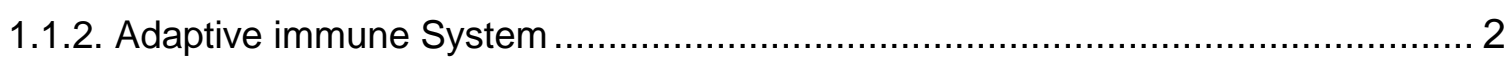

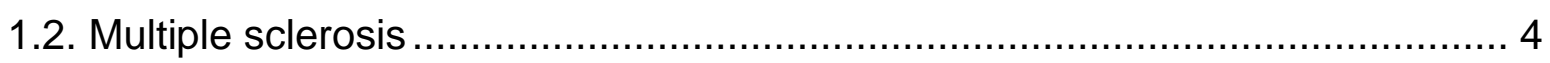

1.3. Experimental autoimmune encephalomyelitis ....................................... 7

1.3.1. Different EAE models in the mouse ...................................................... 8

1.3.2. Immune reactions within the central nervous system ..................................... 9



1.3.3.1. Chemokines and Chemokine receptors in EAE .................................. 12

1.3.4. Role of different lymphocyte populations in EAE ....................................... 13

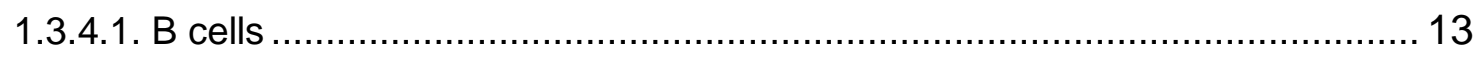

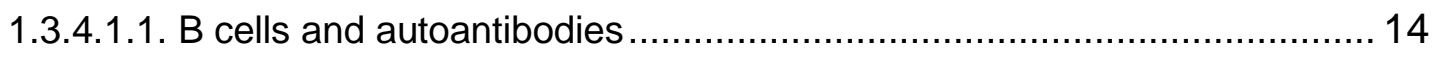

1.3.4.1.2. B cells as antigen-presenting cells................................................ 15



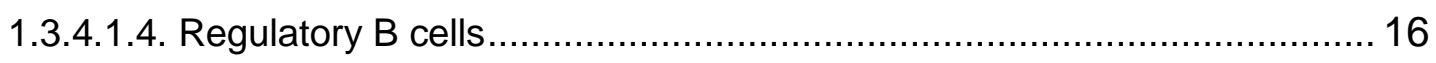

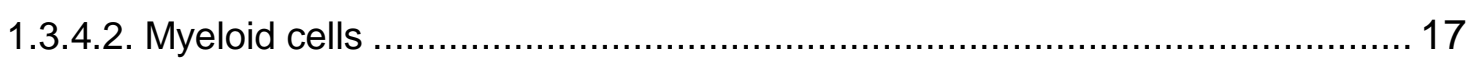



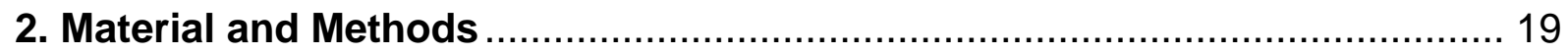

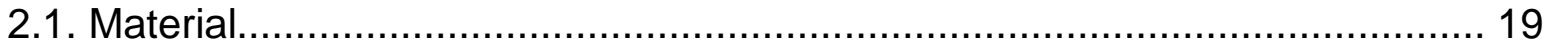

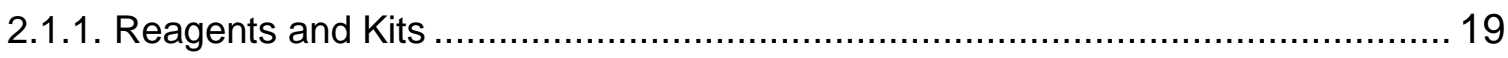

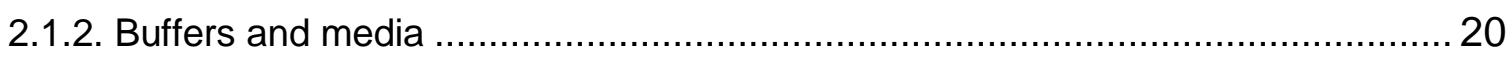

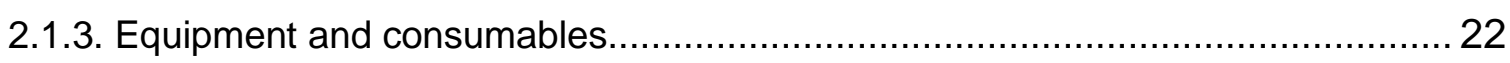

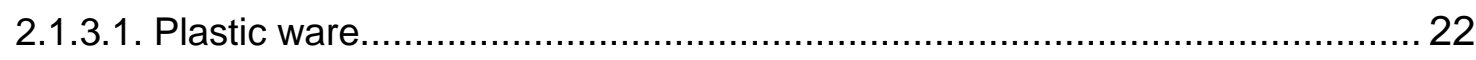





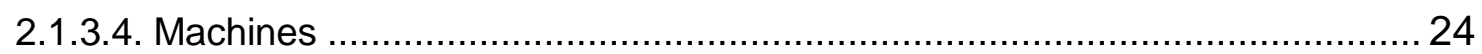

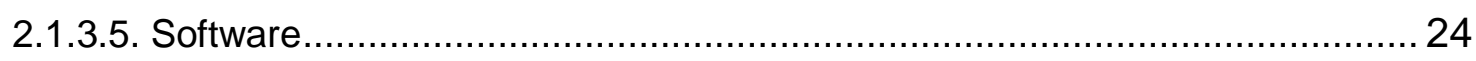


2.1.3.6. Flow cytometry fluorochrome labeled antibodies 25

2.1.3.7. RT-PCR primer and probe sequences ............................................. 26

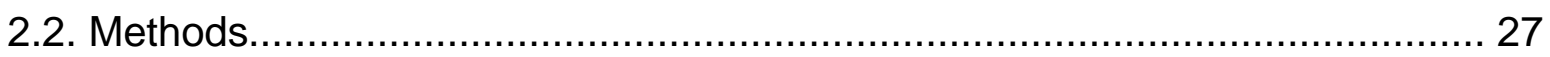

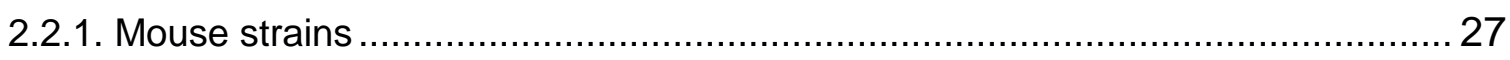

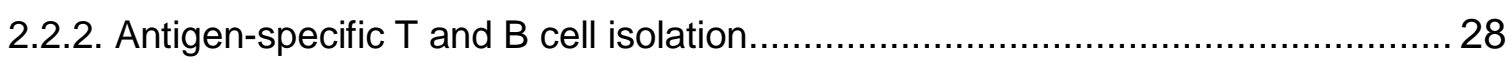



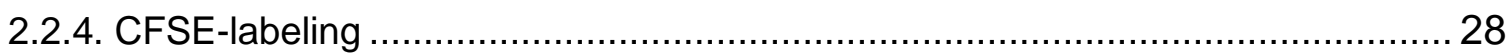



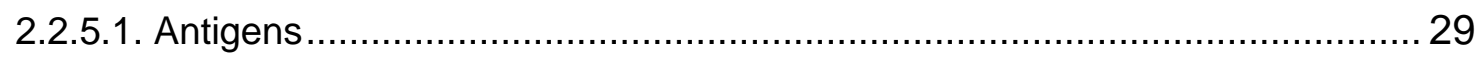

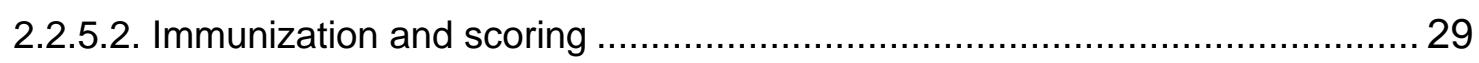

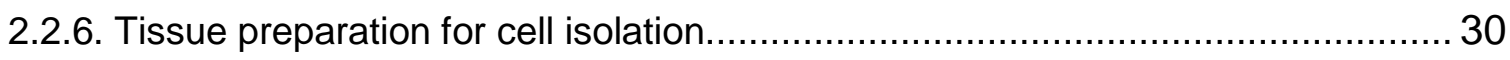

2.2.6.1. Lymphocyte isolation from lymph nodes and spleen ................................ 30

2.2.6.2. Lymphocyte isolation from peripheral blood ............................................ 30

2.2.6.3. Lymphocyte isolation from spinal cord and brain ........................................ 31

2.2.6.4. Isolation of spinal cord microvessel endothelial cells ................................... 31

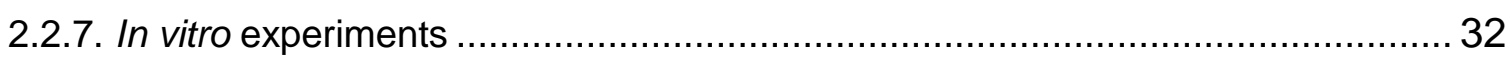



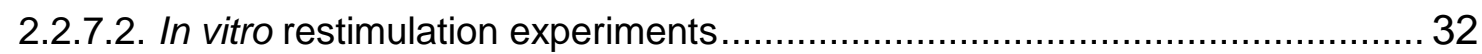

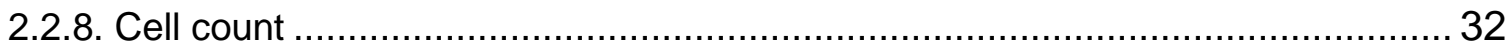

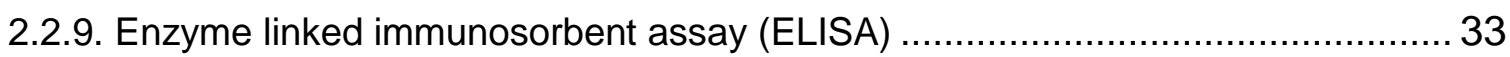

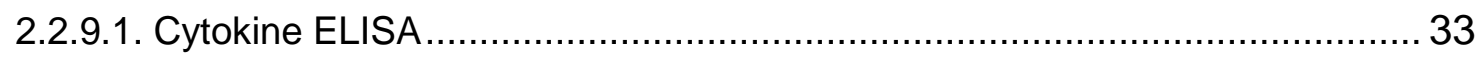



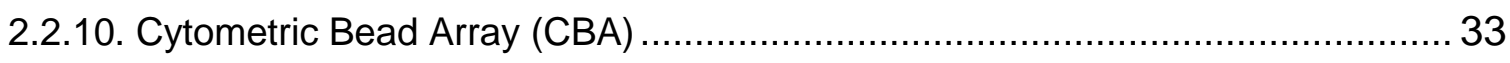

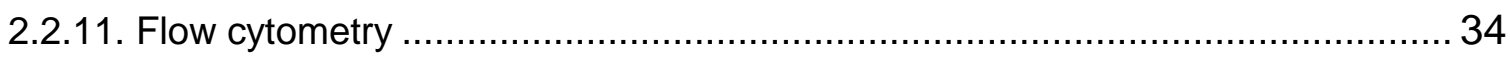

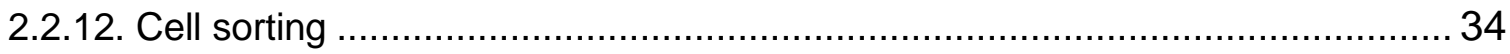

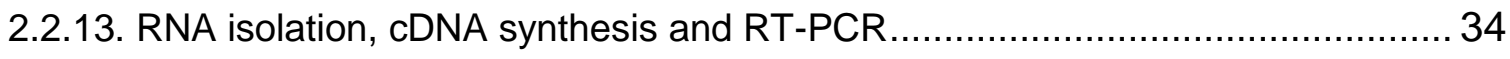

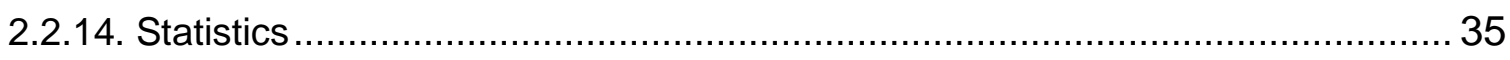

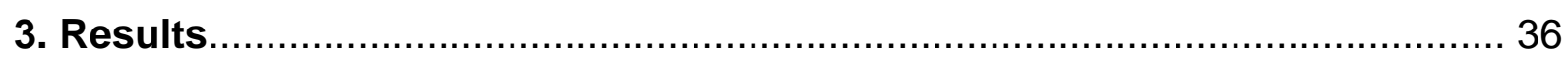

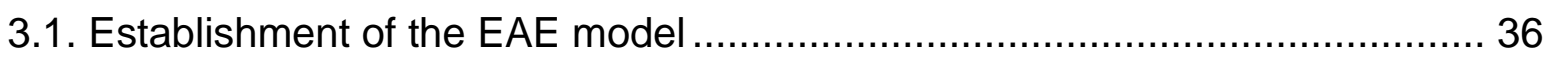

3.1.1. Freshly isolated MOG-specific T cells have a naïve phenotype and exhibit an

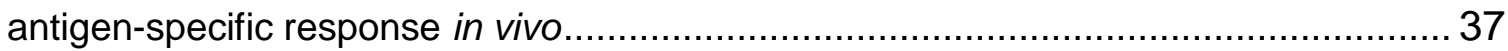

3.1.2. Characterization of the antigen-specific $T$ and $B$ cell interaction in vitro.............. 39

3.1.3. MOG-specific $B$ cells accelerate the disease onset in actively induced $E A E \ldots . . . .40$

3.2. Influence of MOG-specific $B$ cells during the activation phase of EAE ......... 44 
3.2.1. MOG-specific $B$ cells do not enhance antigen-specific $T$ cell proliferation in vivo 45

3.2.2. MOG-specific $B$ cells do not enhance antigen-specific $T$ cell activation in vivo ... 47

3.2.3. MOG-specific $B$ cells do not promote early antigen-specific $T$ cell differentiation in vivo

3.3. Characterization of $T$ cell behavior at the onset of $E A E$

3.3.1. MOG-specific B cells do not influence antigen-specific $T$ cell number and activation before onset

3.3.2. MOG-specific $B$ cells do not promote $T$ cell differentiation before disease onset 54

3.3.3. MOG-specific $B$ cells promote the earlier infiltration of $T$ cells into the CNS........56

3.3.4. Effect of MOG-specific B cells on $T$ cell migration into the CNS

3.3.4.1. MOG-specific T cells show an enhanced expression of CCR6 and even pronounced expression of CXCR4 in the presence of MOG-specific B cells

3.3.4.2. MOG-specific $B$ cells do not influence adhesion molecule expression of $T$ cells

3.4. MOG-specific B cells do not influence mobilization of myeloid cells.

3.5. MOG-specific B cells do not mediate upregulation of adhesion molecules and chemokines by spinal cord microvessel endothelial cells 66

3.6. The antigen-specific interaction of $\mathrm{T}$ and $\mathrm{B}$ cells determine the accelerated disease onset

3.7. MHC-II deficiency of MOG-specific B cells do not exclusively affect their antigen-presenting capacity 70

3.8. The role of antibodies in $M O_{p 35-55}$ induced $E A E$. 72

3.8.1. MOG-specific $B$ cells produce anti-rrMOG IgG upon $M O_{\mathrm{p} 35-55}$ immunization .... 72 3.8.2. Mice with XBP-1 deficient B cells lack anti-rrMOG specific Igs and show a delayed disease onset upon rrMOG immunization. 74

3.8.3. XBP-1-deficient MOG-specific B cells do not accelerate the onset of EAE .76

4. Discussion 80

4.1. Establishment of the EAE model 80

4.2. The role of $M O G$-specific $B$ cells in the activation phase of $E A E$................ 83

4.3. The role of MOG-specific $B$ cells before onset of EAE ........................... 84

4.4. Effect of MOG-specific $B$ cells on $T$ cell migration .................................. 86

4.5. Effect of MOG-specific B cells on myeloid cells................................... 88

4.6. Effect of MOG-specific B cells on spinal cord microvessel endothelial cells... 89

4.7. The antigen-specific interaction of $T$ and $B$ cells determine the accelerated disease onset. 90

4.8. The role of antibodies in $\mathrm{MOG}_{\mathrm{p} 35-55}$ induced $\mathrm{EAE}$ 




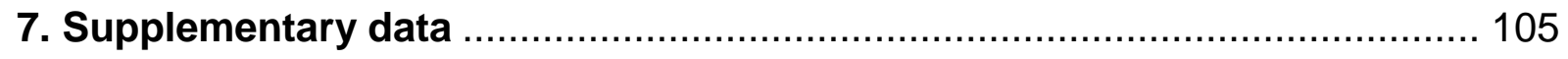

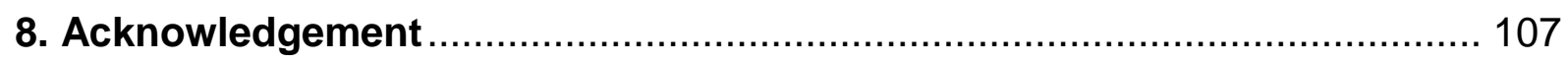

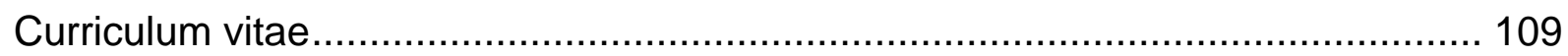

\section{Figure list}

Figure 1: Schematic overview of helper T cell differentiation ................................................ 3

Figure 2: Localization of myelin proteins in the myelin sheath surrounding the neuron ................... 8

Figure 3: T cell-endothelial cell interaction steps in the process of $T$ cell extravasation …….............. 10

Figure 4: Freshly isolated MOG-specific T cells from 2D2 mice have a naïve phenotype ................37

Figure 5: Transferred MOG-specific T cells show antigen-specific proliferation ..............................38



Figure 7: Clinical outcome of MOG-specific T and/or MOG-specific B cell transfer into WT

mice and immunization with $M_{0 G} 3555 \ldots \ldots \ldots$

Figure 8: MOG-specific B cells accelerate the onset of EAE in OT-II and OT-II $x$ NP mice ...........42 42

Figure 9: Schematic overview of experimental design with focus on the activation phase ...............4 44

Figure 10: MOG-specific B cells do not enhance T cell proliferation in vivo $\ldots$

Figure 11: MOG-specific $B$ cells do not influence $T$ cell activation in vivo $\ldots$

Figure 12: MOG-specific $B$ cells do not increase the expression of $\mathrm{TH} 1$ and $\mathrm{TH} 17$ specific

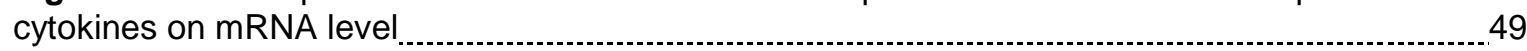



Figure 14: Schematic overview of experimental design with focus on the onset of EAE ..................51

Figure 15: MOG-specific $B$ cells do not influence $T$ cell number or activation at the onset

of the disease

Figure 16: MOG-specific $B$ cells do not trigger T cell differentiation before onset of $E A E$...............5 55

Figure 17: MOG-specific $B$ cells promote the infiltration of MOG-specific T cells into the CNS .......57

Figure 18: No difference in activation status of RFP ${ }^{+}$initial infiltrated T cells

Figure 19: Initial infiltrated T cells utilize the same chemokine receptor for CNS infiltration ...............6 60

Figure 20: MOG-specific B cells influence CXCR4 expression of T cells but have no effect

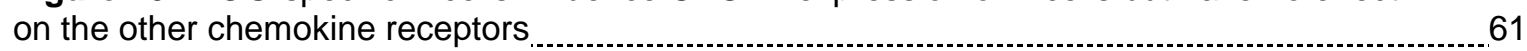

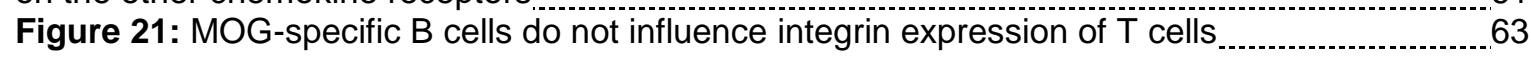

Figure 22: MOG-specific $B$ cells do not influence monocyte mobilization $\ldots$

Figure 23: MOG-specific $B$ cells do not trigger the upregulation of adhesion molecules and chemokines of spinal cord microvessel endothelial cells $\quad 67$

Figure 24: The antigen-specific interaction of $T$ and $B$ cells determine the clinical outcome

of $\mathrm{EAE} \quad 69$

Figure 25: Ablation of $\mathrm{MHC}$-ll of $\mathrm{B}$ cells results in impaired $\mathrm{B}$ cell function $\quad 71$

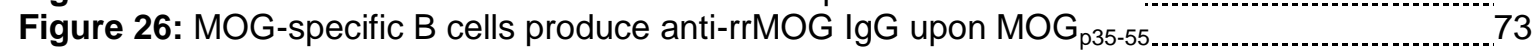

Figure 27: Absence of anti-rrMOG Igs results in a delayed EAE onset

Figure 28: XBP-1 deficiency does not affect the antigen-presenting capacity of $B$ cells ....................... 77

Figure 29: MOG-specific XBP-1-deficient $B$ cells do not accelerate the onset of EAE and do not produce anti-rrMOG specific lgGs 78

Figure S1: NP-specific $B$ cells do not accelerate the onset of EAE

Figure S2: MOG-specific B cells do not inhibit the conversion of naîve MOG-specific T cells

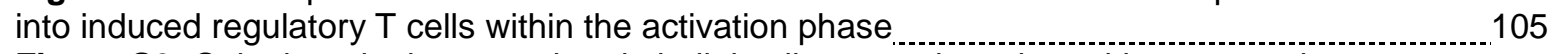

Figure S3: Spinal cord microvessel endothelial cells upregulate chemokine expression upon



Figure S4: In vitro cytokine response of co-cultured antigen-specific lymphocytes

\section{Table list}

Table 1: Flow cytometry antibodies

Table 2: RT-PCR Primer and probes

Table 3: Used mouse strains

Table 4: Influence of cell transfer into OT-II and OT-II $x$ NP mice on EAE susceptibility ..................43

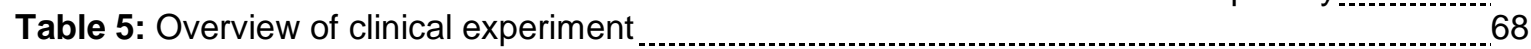




\section{Abbreviations}

APC

antigen-presenting cell

ALCAM

activated leukocyte cell adhesion molecule

BBB

blood-brain barrier

BCR

$B$ cell receptor

CCR

CC chemokine receptor

CCL

CC chemokine ligand

CFA

complete freund's adjuvant

CFSE

carboxyfluorescein succinimidyl ester

CNS

central nervous system

Cre

Cre recombinase

CSF

cerebrospinal fluid

CXCR

CXC chemokine receptor

CXCL

CXC chemokine ligand

d p.i.

days post immunization

EAE

Experimental autoimmune encephalomyelitis

ELISA

Enzyme linked immunosorbent assay

GFP

green fluorescent protein

GM-CSF

granulocyte/macrophage- colony stimulating factor

$\mathrm{h}$

hour

HPRT

hypoxanthine phosphoribosyltransferase 1

ICAM-1

intercellular adhesion molecule 1

IFN $\gamma$

interferon $\gamma$

$\lg$

immunoglobulin

IL-

interleukin-

i.p.

intraperitoneal

i.v.

intravenously

LFA-1

lymphocyte function-associated antigen-1; $\alpha \mathrm{L} \beta 2$

$\mathrm{MHC}$

major histocompatibility complex

$\min$

minute

$\mathrm{ml}$

milliliter

MOG

myelin oligodendrocyte glycoprotein 
$\mathrm{MOG}_{\mathrm{p} 35-55}$

myelin oligodendrocyte glycoprotein aa 35-55, 2D2 TCR epiptope

rhMOG recombinant human myelin oligodendrocyte glycoprotein

rrMOG recombinant rat myelin oligodendrocyte glycoprotein

MS Multiple sclerosis

$\mathrm{NP}$ 4-Hydroxy-3-nitrophenylacetyl hapten

NP-OVAL 4-Hydroxy-3-nitrophenylacetyl hapten fused to OVA protein OVA Ovalbumin

RFP red fluorescent protein

RT-PCR real-time polymerase chain reaction

s.c. subcutanously

TCR T cell receptor

TH cells helper T cells

VCAM-1 vascular cell adhesion molecule 1

VLA-4 very late antigen $4 ; \alpha 4 \beta 1$

XBP-1

$\mathrm{X}$-box binding protein 1 


\section{Abstract}

Although Multiple sclerosis (MS) and its animal model experimental autoimmune encephalomyelitis (EAE) are known as $T$ cell-mediated autoimmune diseases, there is increasing evidence that B cells also play a critical role in their pathogenesis. However, there are conflicting data about the exact role of $B$ cells in the pathogenic process. To study the interaction of MOG-specific $T$ and $B$ cells in the course of actively induced EAE, we developed a model enabling us to track and analyze the cells ex vivo during disease progression. We induced EAE with the encephalitogenic $M \mathrm{OG}_{\mathrm{p} 35-55}$ peptide and found that the transfer of MOG-specific $B$ cells had a disease promoting effect with an accelerated onset. When we focused on the underlying mechanism, we could show that MOG-specific B cells did not enhance MOG-specific $T$ cell proliferation, activation or differentiation during the activation phase or immediately before EAE onset. Moreover, MOG-specific B cells did not enhance T cell activation in the reactivation phase within the CNS in our experimental model. The presence of MOG-specific $B$ cells had no impact on the number of circulating inflammatory myeloid cells. Furthermore, endothelial cells of spinal cord microvessels displayed similar adhesion molecule and chemokine expression levels in the presence of MOG-specific B cells. The accelerated onset was accompanied by an increased number of CNS infiltrated T cells, leading to the speculation that MOGspecific $B$ cells might influence $T$ cell trafficking. The latter is supported by our finding that peripheral MOG-specific $T$ cells showed an enhanced expression of CXCR4 and CCR6 in the presence of MOG-specific B cells. Our clinical data revealed that the presence of activated MOG-specific $B$ cells is critically required for the accelerated disease onset, as activated MOG-unspecific $B$ cells had no effect on the disease onset. When we focused on the mechanism utilized by MOG-specific B cells to promote disease initiation, we found that $B$ cells with an impaired development into antibody secreting plasma cells did not accelerate the disease onset. This led to the conclusion that, even in EAE induced by MOG peptide, B cells promote the initiation of EAE by their secretion of MOG-specific antibodies. 


\section{Introduction}

\subsection{The immune system}

The immune system protects the organism against pathogens and can be divided into an innate and an adaptive immune system. The innate immune response occurs immediately after recognition of the pathogen by macrophages, dendritic cells, natural killer cells and the complement system. The defence mechanisms of the adaptive immune system are mediated by lymphocytes and are characterized by a specific recognition of the pathogen due to an antigen-specific surface receptor and the development of an immunological memory. Under normal circumstances, the processes of an immune response are optimally coordinated and regulated by specialized cells of the immune system to avoid overshooting reactions or to mediate tolerance against non-pathogenic antigens (e.g. self antigens). However, a dysfunction of any factor involved in this process can have fatal consequences for the organism, leading for example to the development of an autoimmune disease like diabetes type I, inflammatory bowel disease or multiple sclerosis.

\subsubsection{Innate immune system}

The innate immune response provides the first line of defence against an invading pathogen. Cells of the innate immune system detect molecular patterns found in most microorganisms by pattern recognition receptors (PRRs) [1]. These microbial structures are referred to as pathogen-associated molecular patterns (PAMPs), whereas molecular patterns from dead or damaged cells are defined as damageassociated molecular patterns (DAMPS) [2]. The most prominent PRRs are the Tolllike receptors (TLRs) [3]. Recognition of the pathogen by cells of the innate immune system either results in its phagocytosis and successful clearing or in the stimulation of the adaptive immune system. Macrophages and dendritic cells act as antigenpresenting cells (APCs). They present antigens, loaded on major histocompatibility complex (MHC) molecules to either $\mathrm{CD}^{+} \mathrm{T}$ cells $(\mathrm{MHC}-\mathrm{I})$ or $\mathrm{CD}^{+} \mathrm{T}$ cells $(\mathrm{MHC}-\mathrm{II})$, which recognize the MHC-antigen complex due to their specific antigen receptor. Macrophages and dendritic cells derive from a common myeloid precursor, the monocyte. Monocytes originate in the bone marrow and are then released into the 
peripheral blood where they appear predominantly as two distinct populations [4]. The short-lived monocyte subset was identified as $C C R 2^{+} L^{2} 6 C^{+} C X_{3} C R_{1}{ }^{-}$. These are commonly referred to as 'inflammatory' monocytes as upon inflammation they were found to be immediately recruited into the tissue in a CCR2-dependent manner $[5,6]$. In contrast, the CCR2 ${ }^{-} y 6 \mathrm{C}^{l 0} \mathrm{CX}_{3} \mathrm{CR}_{1}{ }^{+}$monocyte population have a longer half-life and are considered as 'resident' monocytes. They migrate into various tissues under non-inflammatory conditions in a $\mathrm{CX}_{3} \mathrm{CR}_{1}$-dependent manner [4]. $A$ third very small monocyte population was identified as $\mathrm{CCR} 7^{+} \mathrm{CCR} 8^{+}$and it was shown that they emigrate to lymph nodes where they appear as monocyte-derived dendritic cells [7].

\subsubsection{Adaptive immune System}

$T$ and $B$ lymphocytes are the main players of the adaptive immune system, which can be divided into two functional parts: the cellular and the humoral system. B cells mediate humoral immunity by their production and secretion of high-affinity antibodies and are part of the cellular system due to their antigen-presenting capacity and their secretion of cytokines. One main characteristic of cells from the adaptive immune system is their specific antigen receptor. The antigen receptor specificity is determined by the antigen-binding site of the T cell receptor (TCR) and the B cell receptor (BCR). The enormous range of specificity is achieved by somatic recombination of the DNA which encodes the different segments of the receptor.

T cells can be further divided into $\mathrm{CD}^{+}$(co-receptor) or $\mathrm{CD}^{+}$(co-receptor) T cells. Both $T$ cell subtypes share the feature that they only recognize an antigen when it is bound to a MHC molecule on the surface of an antigen-presenting cell. Due to the different co-receptors, that interact with $\mathrm{MHC}$ molecules on the $\mathrm{APC}, \mathrm{CD} 4^{+} \mathrm{T}$ cells recognize the antigen in the context of $\mathrm{MHC}-\mathrm{II}$ and $\mathrm{CD}^{+}$in the context of MHC-I. The major function of $\mathrm{CD}^{+} \mathrm{T}$ cells is the elimination of intracellular pathogens by cytolysis of the infected cell and they are therefore called cytotoxic T cells. CD4 ${ }^{+} \mathrm{T}$ cells are also known as helper T cells (TH cells). They show a high phenotype plasticity and, depending on the surrounding cytokine milieu, they develop into different helper $\mathrm{T}$ cells subtypes as schematically depicted in Figure 1. 


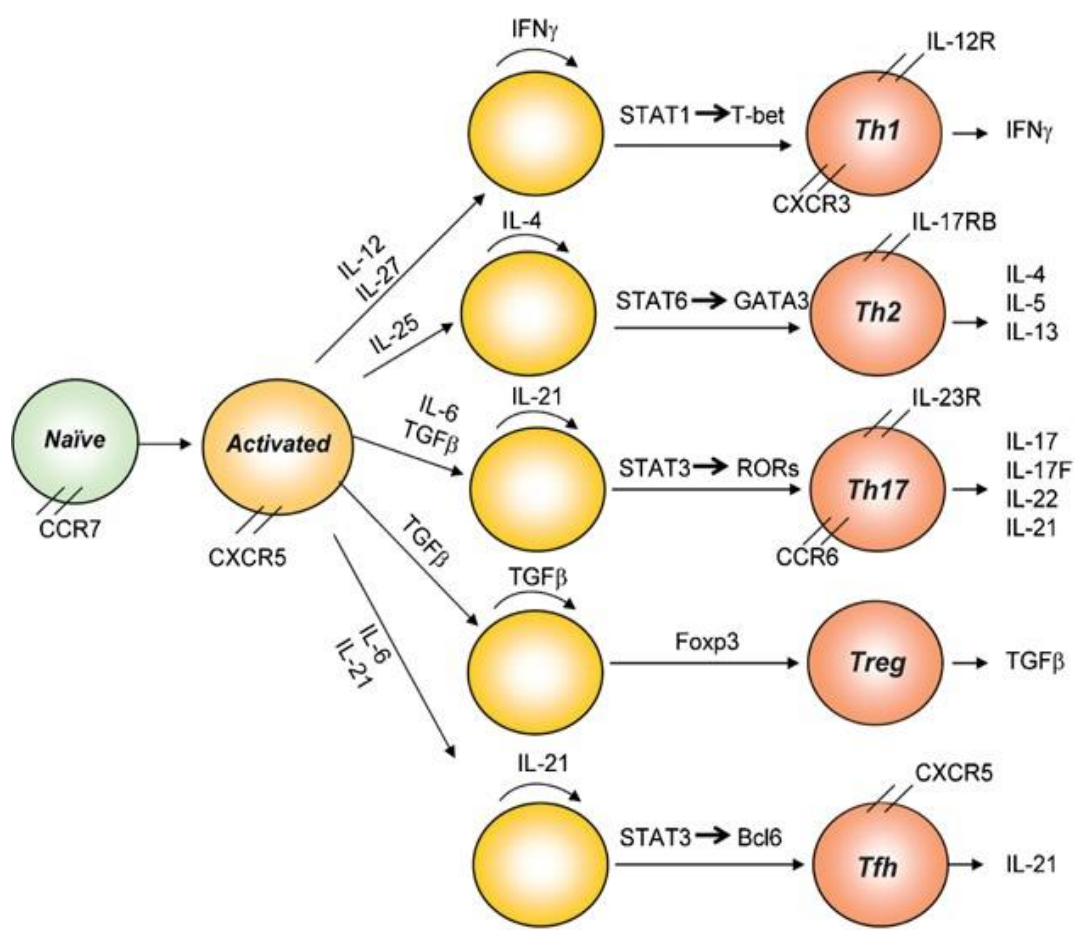

Figure 1: Schematic overview of helper $\mathrm{T}$ cell differentiation. Upon naïve $\mathrm{T}$ cell (blue) activation by antigen encounter the T cells develop either into $\mathrm{TH} 1, \mathrm{TH} 2, \mathrm{TH} 17$, induced regulatory $\mathrm{T}$ cells (iTreg cells) or T follicular helper cells (Tfh) depending on the cytokine milieu. The different CD4 ${ }^{+}$ $\mathrm{T}$ cell subsets are characterized by their main transcription factors and their secreted cytokine(s). Figure modified from [8].

Together with antigen presentation, the production of interleukin-12 (IL-12) by activated macrophages and dendritic cells induces the differentiation of naïve $T$ cells into TH1 cells $[9,10]$. IL-12 binds to the IL-12 receptor on the T cell surface which activates a signal transducer and activator 4 (STAT4) signaling pathway resulting in the transcription of the transcription factor T-box expressed in $T$ cells ( $T$ - bet) and IFN $\gamma$ [11]. In a positive feedback loop, IFN $\gamma$ binds to its receptor on the $T$ cells surface, thereby activates the signal transducer and activator 1 (STAT1) signaling pathway and enhance T-bet and its own expression [12]. In contrast, the cytokine IL25 triggers the differentiation of naïve T cells into IL-4, IL- 5 and IL-13 producing TH2 cells [13]. IL-4 then leads to the activation of the STAT6 pathway [14] resulting in an upregulation of the transcription factor GATA-3 enabling the expansion of TH2 cells [15]. The differentiation of naive T cells into TH17 cells is triggered by either the cytokines IL-6 or IL-21 in combination with transforming growth factor beta (TGF $\beta$ ), and the transcription factor orphan nuclear receptor ROR $\gamma$ t is critical for $\mathrm{TH} 17$ cell development [16-18]. The cytokines IL-1 $\beta$ and IL-23 are crucial for the maintenance 
of the TH17 phenotype $[19,20]$. TH17 cells produce a variety of effector cytokines like IL-17A, IL-17F, IL-21, IL-22, TNFa and granulocyte/macrophage-colony stimulating factor (GM-CSF) [21]. The cytokines TGF $\beta, \mathrm{IL}-2$ and IL-10 induce the differentiation of naïve $\mathrm{CD} 4^{+} \mathrm{T}$ cells into induced regulatory $\mathrm{T}$ cells (iTreg cells) by the induction of their critical transcription factor FOXP3 [22-24]. In contrast, natural regulatory $T$ cells (nTreg cells) develop in the thymus during the process of negative selection [25]. Regulatory $T$ cells play an important role in mediating tolerance to selfantigens and establishing immune homeostasis by e.g. suppression of $\mathrm{T}$ cell proliferation or their differentiation into effector $T$ cells [26, 27]. $T$ follicular helper cells (Tfh cells) provide help for B cells to induce germinal center formation and are mainly characterized by their surface expression of CXCR5 and ICOS. The transcriptional repressor Bcl6 was shown to be critical for the differentiation of naïve T cells into Tfh cells upon IL-21 and IL-6 stimulation [28-30].

\subsection{Multiple sclerosis}

Multiple sclerosis is an autoimmune disease affecting the central nervous system (CNS). An autoimmune disease is defined by the presence of an immune response directed against self- antigens leading to the activation and amplification of selfreactive cells which results in organ-specific inflammation and tissue disruption. Multiple sclerosis is considered as a T-cell mediated autoimmune disease, where effector mechanisms of autoreactive myelin-specific $T$ cells are directed against myelin antigens leading to myelin sheaths disruption (demyelination) and axonal damage.

Multiple sclerosis is the most common inflammatory disease of the central nervous system (CNS) affecting more than 2 million people worldwide, but it is more common among Caucasians. First clinical signs typically appear between 20 to 40 years of age and females show a higher susceptibility than males. Typical clinical symptoms are paralysis and impaired vision and cognitive function [31]. There are different forms of MS regarding the clinical course of disease. The most common form is the relapse-remitting MS one (RR-MS). Patients of this MS form suffer from periods of acute attacks alternating with periods of recovery to near normal neurological function. In some cases, the RR-MS form turns into the secondary-progressive MS 
(SP-MS) form, which is accompanied by irreversible progression of clinical disability after a relapse. Patients of primary-progressive MS (PP-MS) and progressiverelapsing MS (PR-MS) suffer a clinical progression from the initiation of the disease. The PP-MS course shows a gradual increase of disease symptoms, whereas PR-MS disease course is accompanied by acute relapses [32].

The etiology of MS is a central question of research. A high number of factors are associated with MS susceptibility. Environmental triggers, genetic risk factors and infectious diseases are those predominantly discussed. Environmental risk factors are for example reduced Vitamin D levels, smoking or special diets (association of high salt intake and its effects on the disease have only been investigated in the MS animal model EAE until now) [33-38]. In addition, infectious agents like the EpsteinBarr virus (EBV) and, related to this, the hygiene hypothesis, are also considered to increase the susceptibility for MS [39]. Observations from population-based studies of twins and an elevated frequency of the disease in relatives from affected patients argue for a genetic predisposition for MS [40]. Genome-wide association studies revealed several risk loci associated with MS and almost all of them are related to the immune system. The most prominent of these is the HLA (Human Leukocyte Antigen) allele $D R B^{\star} 11501$ - it was shown that single nucleotide polymorphism (SNP) in this allele has the strongest association with MS [41]. Moreover, there were risk loci of genes identified encoding cytokine receptors (IL-2RA, IL-7RA) or costimulatory molecules (CD80, CD86) [41], suggesting an immune dysfunction as a potential cause for MS.

In MS, tissue damage occurs predominantly in the white matter of the brain and the spinal cord. Focal inflammatory demyelinated lesions are characterized by immune cell infiltrates and results in axonal damage and gliosis. In the progressive stages of the disease (SP-, PP-MS), also cortical demyelination can be detected [42]. Histopathological studies reveal a high heterogeneity in lesional profiles. However, by investigating actively demyelinating lesions of biopsy and autopsy cases, four different patterns of demyelination were defined. The presence of infiltrating $T$ cells and macrophages is common in all cases, but they differ for example in the localization of the demyelinated plaques or the presence of immunglobulins and complement [43]. However, the reason for the different patterns is not clear and it is not a direct evidence for the pathogenic mechanism. 
Several cellular dysfunctions have been uncovered in MS patients. Interestingly, myelin- specific T cells are not exclusively detected in the blood of MS patients, but also in healthy individuals. However, it was shown that myelin-specific T cells isolated from the blood and especially from the CSF of MS patients persist in a different activation status than $T$ cells from healthy controls leading to a higher sensitivity upon antigen encounter [44-47]. Also regulatory cells from MS patients reveal an impaired cellular function. The suppressive capacity of regulatory $T$ cells from MS patients is decreased leading to a loss of regulatory function [48, 49]. There is evidence that also pro-inflammatory and regulatory $B$ cells are critically involved in the progression of MS. Ectopic lymphoid follicle-like structures containing a high number of $B$ cells and plasma cells were found in the meninges of SP-MS patients [50,51] and the presence of oligoclonal bands in the CSF of almost all MS patients can be used as a supportive diagnostic criteria [52]. In addition, autoantibodies against MOG are directly associated with myelin damage [53, 54]. Peripheral B cells isolated from blood of RR-MS patients exhibit an augmented pro-inflammatory cytokine response in comparison to B cells from healthy controls [55, 56]. Clinical studies with Rituximab, a monoclonal antibody which selectively targets and depletes $\mathrm{CD} 2 \mathrm{O}^{+} \mathrm{B}$ cells, reveal beneficial effects in the treatment of MS. In a phase 2 study, relapseremitting multiple sclerosis patients show reduced lesions compared to placebo treated patients and the proportion of Rituximab treated patients with relapses was reduced [57]. The treatment of RR-MS patients results in a reduced $T$ and $B$ cell number in the cerebrospinal fluid at six months post treatment and an accompanied reduction of serum antibodies against myelin oligodendrocyte glycoprotein and myelin basic protein in some patients [58]. However, the beneficial effects observed with Rituximab therapies are in contrast to clinical trials with another B cell-depleting drug called Atacicept. Atacicept is a humanized recombinant fusion protein containing the extracellular ligand- binding portion of the human transmembrane activator and calcium modulator and cyclophilin-ligand interactor (TACl) receptor fused to a recombinant Fc domain of human IgG. The receptor binds the cytokines Blymphocyte stimulator (BLyS) and a proliferation-inducing ligand (APRIL) -cytokines involved in $B$ cell differentiation, maturation, and survival, and thus inhibits their action on B cells $[59,60]$ Clinical studies with Atacicept reveal increased clinical disease activity [61]. The underlying reasons for the observed worsening of clinical symptoms are not yet clarified in detail, but it is considered that Atacicept, in contrast 
to Rituximab, also targets and affects plasma cells [62], which were recently shown to play a regulatory role in the pathogenesis of MS. Regulatory B cells are a recently discovered $B$ cell subpopulation and their contribution to CNS autoimmune disease was until now mainly investigated in the MS mouse model EAE (regulatory $B$ cells are described in detail in chapter 1.3.4.1.4.). However, in mice as well as in humans, they exert their regulatory function mainly by the production of IL-10 and IL-35 and it was shown that B cells from MS patients exhibit a reduced production of IL-10 [63].

\subsection{Experimental autoimmune encephalomyelitis}

The animal model experimental autoimmune encephalomyelitis (EAE) mimics several aspects of the human autoimmune disease Multiple sclerosis. Most of the EAE studies were performed in rodents like mice and rats but it can also be induced in other animals like non-human primates $[64,65]$. In susceptible mouse strains, EAE can be induced in two ways- either by adoptive transfer of myelin antigen-primed encephalitogenic $T$ cells (transfer EAE) or by immunization with a myelin antigen emulsified in adjuvant (active EAE). For transfer EAE, antigen-specific $\mathrm{T}$ cells are obtained by immunization with a respective antigen following by in vitro $\mathrm{T}$ cell restimulation with antigen and cytokines pushing the $\mathrm{T}$ cells into $\mathrm{T}$ helper cell differentiation. It was shown that $\mathrm{TH} 1$ and $\mathrm{TH} 17$ cells, but not $\mathrm{TH} 2$ cells, can efficiently induce EAE in different mouse strains [21, 66-70]. The critical cytokine of TH1 cells in the context of EAE is IFN $\gamma[66,67,69]$. TH17 cells produce a wide range of cytokines [21], whereas only IL-17A and GM-CSF seem to play a critical role in EAE [69, 71, 72]. The myelin proteins predominantly used to induce EAE are the myelin oligodendrocyte glycoprotein (MOG), proteolipid protein (PLP) and the myelin basic protein (MBP) and their respective $\mathrm{T}$ cell relevant peptide epitopes (e.g. $\mathrm{MOG}_{\mathrm{p35-55}}, \mathrm{PLP}_{139-151}$ ) [73-75]. An overview of their location within the myelin sheath is depicted in Figure 2. 


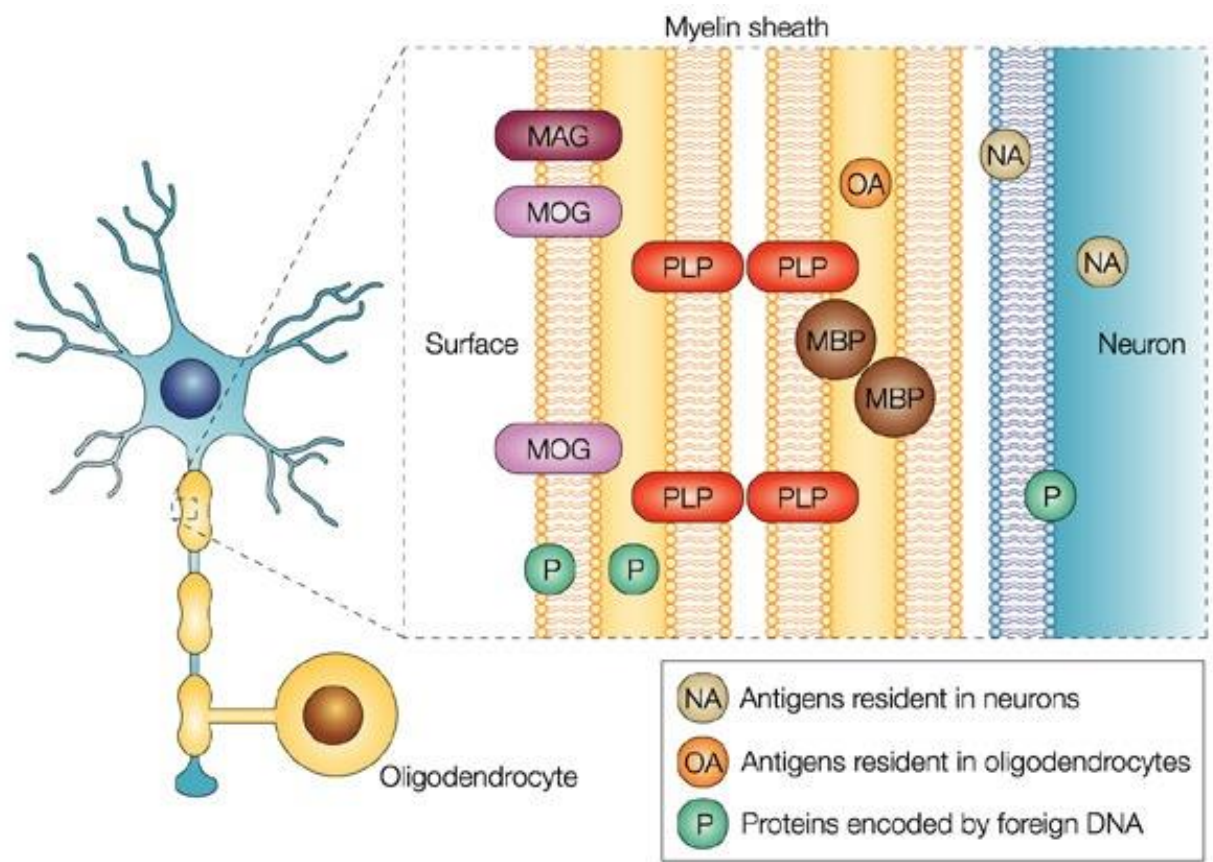

Figure 2: Localization of myelin proteins in the myelin sheath surrounding the neuron. Myelin-associated protein (MAG) and myelin oligodendrocyte glycoprotein (MOG) are located on the outer surface of the myelin sheaths, proteolipid protein (PLP) and myelin basic protein (MBP) are located in the inner layers of the myelin sheaths [76].

\subsubsection{Different EAE models in the mouse}

EAE and MS are both autoimmune in nature. The physical disabilities are the consequence of mononuclear cell infiltration into the central nervous system leading to demyelination, axonal damage and tissue destruction. However, the animal model does not resemble the full spectrum of the human disease. To overcome this problem several EAE models have been established with each mimicking different characteristics of the human disease. Differences of the clinical phenotypes are due to the different genetic repertoire of each susceptible mouse strain, especially influenced by different MHC-haplotypes. Immunization of C57BI/6 mice with either the MOG protein or with the $T$ cell epitope $M_{0} G_{p 35-55}$ as well as the adoptive transfer of MOG-specific TH1 or TH17 cells leads to a chronic form of EAE with inflammatory foci predominantly present within the spinal cord rather than within the brain [69, 75]. MOG-specific $T$ cell receptor transgenic mice (2D2 mice) can develop EAE spontaneously but with a very low incidence (4\%) [77], but the cooperation of MOGspecific $T$ and $B$ cells in a double-transgenic mouse model led to the spontaneous development of EAE with an incidence of $59 \%[78,79]$. The relapse-remitting course 
of MS, the most common form of the disease, can be resembled by the adoptive transfer of PLP 139-151 primed T cells or by immunization of SJL/J mice with PLP. These mice develop EAE with a relapse-remitting course [74, 80]. Additionally, a $T$ cell receptor transgenic mouse model on the SJL/J background spontaneously develop EAE with a relapse-remitting disease course [81]. In most EAE models the infiltration of cells into the CNS is restricted to the spinal cord rather than to the brain. However, there are some rodent models where immunization leads to brain inflammation mimicking the predominant MS CNS histopathology [82, 83].

\subsubsection{Immune reactions within the central nervous system}

The central nervous system is often considered as an immune privileged organ e.g. due to its special microvessel endothelial cells restricting cell entry or the absence of lymphatic vessels. However, with increasing investigation it became clear, that the CNS is not completely devoid of immune reactions. CNS resident cells like microglia and astrocytes express MHC-II as well as co-stimulatory molecules, but also a low number of perivascular and meningeal macrophages [84] could be found under healthy physiological conditions. Activated $T$ cells injected into the periphery were also found to be able to cross the blood brain barrier [85-87]. Additionally, the rejection of tissue transplants into the brain of immunologically primed recipients in contrast to immune deficient recipients emphasize the potential for immune reactions within the CNS [88].

The CNS is surrounded by a special membrane structure termed meninges. This structure is composed of three different membrane layers- the dura mata, which is in direct contact to the skull and the arachnoid mater which together with the pia mater define the subarachnoidal space comprising the cerebrospinal fluid (CSF). The CSF is produced within the ventricles of the brain by the choroid plexus and circulates through the brainstem around the outer surface of the spinal cord and is crucial for CNS metabolism and homeostasis. The subarachnoidal space is crossed by an artery network whose capillaries penetrate into the CNS parenchyma. An endothelial basement membrane on the vessel wall side and the glia limitans enclose the perivascular space. The CNS microvessels are lined by special endothelial cells also defined as blood-brain barrier (BBB). The endothelial cells are connected by tight 
junctions limiting paracellular entry of soluble factors. The glia limitans surrounding the endothelial cells play an important role in maintaining the BBB integrity $[89,90]$.

\subsubsection{T cell migration in $\mathrm{EAE}$}

Under physiological conditions, the frequency of cell migration into the CNS is very low. However, under some circumstances as in the autoimmune disease MS or its animal model EAE, the infiltration of e.g. $T$ cells and mononuclear phagocytes is augmented causing disruption of the BBB integrity, further cell invasion and manifestation of inflammation within the CNS. Generally, there are some possible sites for T cells to enter the CNS- across the choroid plexus into the CSF, from the blood into the subarachnoidal space or into the perivascular space. Even though, the exact molecular mechanisms utilized by $T$ cells to interact and finally to overcome the BBB does not seem to be clarified in detail, it is clear that the extravasation of T cells is a multi-step process, as depicted in Figure 3

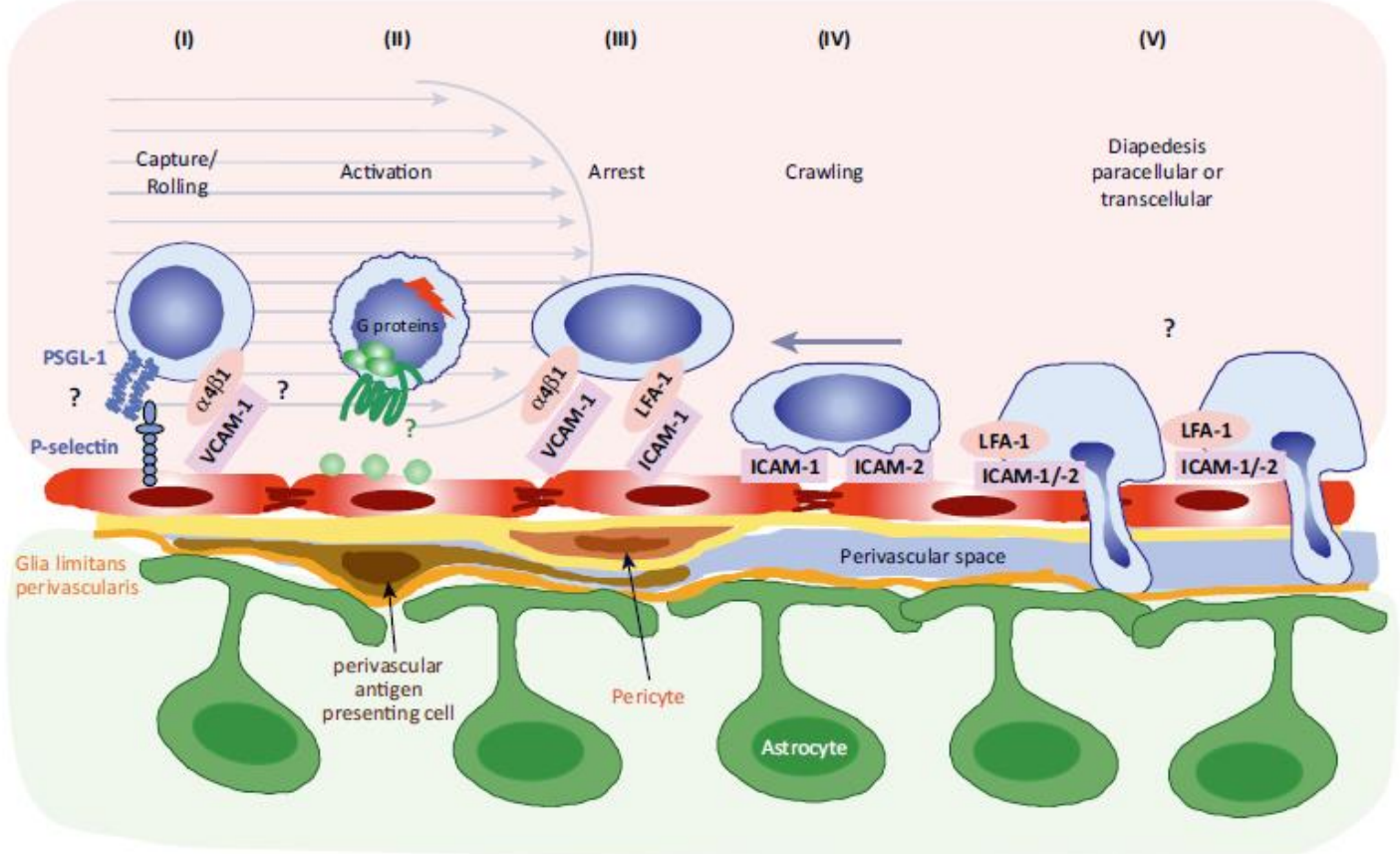

Figure 3: $T$ cell-endothelial cell interaction steps in the process of $T$ cell extravasation. T cell invasion can be divided into different steps each underlying different molecular mechanisms: 1) capture/ rolling, 2) activation, 3) firm adhesion (arrest), 4) crawling and 5) transmigration [91].

The initial interaction of T cells with CNS microvessel endothelial cells is mediated by selectins and integrins and their respective counterparts expressed by endothelial 
cells. In EAE, the role of P-selectin glycoprotein ligand-1 (PSGL-1) expressed by $T$ cells is controversial. Whereas PSGL-1 expression play a critical role for T cells to home into inflamed skin, it rather seems to be dispensable for EAE development [9295]. The interaction of alpha 4 beta 7 integrin ( $\alpha 4 \beta 1$; very late antigen 4 (VLA-4)) expressed by $T$ cells with vascular cell adhesion molecule 1 (VCAM-1), expressed by endothelial cells, is one of the most critical factors involved in EAE development as functional interference results in complete absence of EAE symptoms [70, 96, 97]. VLA-4 expression by $T$ cells mediate the initial capture as well as the firm adhesion of T cells to endothelial cells $[98,99]$. Due to its critical involvement, the VLA-4-VCAM1 interplay arose as a promising target for therapeutical treatment of MS. The humanized monoclonal antibody natalizumab binds to $\alpha 4 \beta 1$ and the treatment of RRMS patients shows beneficial effects $[100,101]$. The initial capture brings the chemokine receptor expressing $T$ cells in close proximity to chemokines secreted by endothelial cells (chemokine receptors and chemokines are described in detail in chapter 1.3.3.1.). The chemokine receptor signaling in turn leads to the activation of integrins resulting in its conformational changes and clustering which increases their affinity and avidity $[102,103]$. Consequently, the initial capture changes into a firm arrest enabling the $T$ cell to further interact with the endothelial cells. T cells express the alpha $L$ beta 2 integrin ( $\alpha \mathrm{L} \beta 2$; lymphocyte function-associated antigen-1 (LFA-1)) which interacts with intercellular adhesion molecule 1 (ICAM-1) on endothelial cells [104]. In vitro and in vivo studies under healthy conditions reveal that the LFA-1ICAM-1 interaction play a role in the transmigration step $[105,106]$. However, under inflammatory conditions its role is controversial and difficult to interpret because LFA1 also plays a role in T cell activation [107]. Therefore, the results of immunization of LFA-1-deficient mice range from a higher EAE susceptibility to milder course of disease $[108,109]$. Similar results were obtained by ICAM-1 neutralization in the rat system [110, 111]. Activated leukocyte cell adhesion molecule (ALCAM) is an adhesion molecule which is upregulated on CNS vessels of active MS lesions and by spinal cord cells during active EAE. Blockade of ALCAM ameliorate EAE symptoms and is accompanied by a reduced number of infiltrating cells but does not affect $T$ cell activation in the periphery. Therefore, it presumably plays a role in $\mathrm{T}$ cell trafficking into the CNS rather than in interfering with T cell activation [112].

Once $\mathrm{T}$ cells cross the BBB, they localize within the leptomeningeal space or the perivascular space, where they experience a second auto-antigen encounter by 
resident APCs leading to their reactivation $[113,114]$. The local reactivation is a crucial step to enable $T$ cell infiltration into the CNS parenchyma and to cause disease. It was shown, that also activated myelin-unspecific $T$ cells can enter the CNS tissue, even in equal numbers to that observed with myelin-specific T cells, but due to their failed reactivation they are not able to cause clinical symptoms [115, 116]. The reactivation of $T$ cells is accompanied by an upregulation of various cytokines and chemokines leading to the attraction of not only further T cells but also other cells like macrophages and neutrophils [117].

\subsubsection{Chemokines and Chemokine receptors in EAE}

Several publications suggest an involvement of chemokines and their respective receptors during the course of EAE. Upon inflammatory conditions, TH1 cells upregulate the chemokine receptors CXCR3 and CCR5 $[118,119] . \mathrm{CXCR}^{+}$and $\mathrm{CCR}^{+} \mathrm{T}$ cells have been found in the CNS of EAE animals or in MS patients suggesting a role for mediating CNS trafficking in CNS inflammation [120-122]. However, under experimental conditions, the roles of CXCR3 and CCR5 and their respective ligands are not clearly defined. Whereas active immunization of CXCR3 knockout mice results in a higher EAE severity, as well as in a more severe chronic phase, CXCR3 blockade in an adoptive transfer model leads to a milder disease course and a delayed onset $[120,121,123,124]$. The CXCR3 ligand IFN- $\gamma$-Inducible Protein-10 (IP-10, CXCL10) becomes upregulated during the course of EAE, but similar to observations with CXCR3 deficiency, the neutralization of IP-10 in adoptive transfer EAE reduces clinical signs, whereas in actively induced EAE it rather exacerbates the disease, presumably due to an additional role in $\mathrm{T}$ cell activation [125-127]. Immunization of either CCR5- or macrophage-inflammatory protein-1a (MIP-1a) knockout mice, a CCR5 ligand which is upregulated during EAE, does not influence the clinical disease, but MIP-1 $\alpha$ neutralization in an adoptive transfer EAE model prevents induction of acute and relapsing EAE without affecting $T$ cell activation [128, 129]. Beside inflammatory chemokines, cells from the CNS also upregulate the expression of homeostatic lymphoid chemokines like CCL19, CCL21 and CXCL12 suggesting a role for chemoattraction of $T$ cells during inflammation $[103,130]$. In line with this, CXCR4 ${ }^{+} \mathrm{T}$ cells are present within the inflamed CNS [120]. In healthy tissue, CXCL12 is expressed by spinal cord endothelial cells at the basolateral surface where it is suggested to retain CXCR4-expressing cells in the 
perivascular space inhibiting them to migrate into the parenchyma. In this context, CXCR4 antagonization with AMD3100 during EAE leads to worsening of clinical signs, whereas CXCR4 antagonization with a CXCL12 mutant (CXCL12 ${ }_{\mathrm{P} 2 \mathrm{G} 2)}$ ameliorates EAE in another mouse model $[120,130]$. In contrast to TH1 cells, TH17 cells predominantly express the chemokine receptor CCR6 and its ligand CCL20 was shown to attract TH17 cells to various sides of inflammation [131-134]. CCR6 knockout mice are resistant to actively-induced EAE, but this resistance is abrogated by transfer of CCR6-sufficient T cells, suggesting a role for CCR6 in TH17 cell traffic into the CNS. CCR6-sufficient T cells enter the CNS through CCL20-expressing epithelial cells of the choroid plexus, from where they initiate inflammation, which in turn recruits a second wave of T cells in a CCR6-independent manner [135].

\subsubsection{Role of different lymphocyte populations in EAE}

Although myelin-specific $T$ cells have been identified as the crucial cells to initiate CNS autoimmunity in various animal models of MS, it is also considered that other cell types of the adaptive and of the innate immune systems are also critically involved in the initiation and progression of EAE and MS.

\subsubsection{B cells}

Although Multiple sclerosis and its animal model experimental autoimmune encephalomyelitis are T-cell mediated autoimmune diseases, the role of B cells in its pathologies have come into focus within the past decade. Beneficial effects of Rituximab therapy, the presence of oligoclonal bands in the cerebrospinal fluid of MS patients as well as beneficial effects of therapeutic plasma exchange support the hypothesis of B-cell contribution to MS [136].

There are many publications reporting on studies into the role of $B$ cells in EAE. They differ in the model used and in the antigens used. C57bl/6 WT and B-cell deficient mice ( $\mu M T$; [137]) are equally susceptible to MOG peptide-induced EAE, but when immunized with human recombinant MOG protein (rhMOG) B cell deficient mice are protected from $E A E$, which suggests a pronounced role for $B$ cells in human MOG protein induced EAE $[138,139]$. In addition, the ability of rhMOG to induce EAE in $B$ cell deficient mice was restored by the injection of rhMOG-primed $B$ cells (but not by injection of naïve WT or unspecifically activated B cells) prior to disease induction but 
with a later onset and milder disease course compared to WT control group [140]. This observation was confirmed by others and it was additionally shown that the immunization with recombinant mouse MOG ( $\mathrm{rmMOG}$ ) is also $B$ cell independent $[139,141]$. However, the $B$ cell depletion by an anti-CD20 depletion antibody at the onset of MOG peptide-induced EAE results in a faster recovery, suggesting also a role for $B$ cells in MOG peptide induced EAE [56, 142]. The contribution of $B$ cells to the development of EAE is also emphasized in spontaneous EAE models. Only 4\% of the MOG-specific T cell receptor transgenic mice (2D2 mice, [77]) on the C57bl/6 background develop spontaneous EAE. However, when they are crossed with MOGspecific Ig heavy-chain knock-in mice (Th mice, [143]) 59\% of the animals develop spontaneous EAE accompanied by a higher clinical score, earlier day of onset and a higher number of inflammatory foci $[78,79]$. A second model using MOG-specific T cell receptor transgenic mice (1C6, [144]) on the NOD background shows a rare spontaneous EAE development, but when crossed with Th mice, $45 \%$ of male and $79 \%$ of female mice develop EAE spontaneously.

\subsection{B cells and autoantibodies}

The mechanism utilized by $B$ cells to contribute to the pathogenesis of EAE is controversially discussed. The main characteristic of $B$ cells is their ability to produce and secrete antibodies. Due to the occurrence of oligoclonal bands in the cerebrospinal fluid in almost all MS patients [145], beneficial plasma exchange therapies [136] and the contribution of autoantibodies to other autoimmune diseases [146-149], it has been suggested that autoantibodies against myelin antigens may play a role in MS and EAE pathology. Immunization of MOG-specific B cell receptor transgenic mice (Th, [144]) either on the C57BI/6 or on the SJL background results in an accelerated and exacerbated disease course compared to non-transgenic littermates emphasizing a role for anti-MOG-specific antibodies [143]. Studies with MOG peptide-induced EAE of $B$ cell deficient mice reveal a dispensable role for $B$ cells and for autoantibodies against myelin antigens [140, 150]. Double-transgenic mice and also mice immunized with rhMOG or rMOG have high anti-MOG IgG titers $[78,141,151]$ and i.v. sera transfer into $B$ cell deficient mice at the time point of immunization restores the susceptibility to EAE to similar levels observed in WT mice [140]. However, the transfer of anti-MOG 8-18C5 (m-monoclonal antibody against MOG) starting at the onset of EAE only partially restores EAE susceptibility in B cell 
specific MHC-II knockout animals [139], whereas when administered into SJL/J mice after the animals recovered from an attack, it induces severe relapses [152]. In a transgenic model where $B$ cells express the membrane MOG-specific immunoglobulin, but are incapable of secreting it, immunization either with MOG peptide, rmMOG or rhMOG results in the same onset and clinical severity, suggesting a dispensable role for $\alpha-M O G$ antibodies in this experimental setting [139]. The contribution of $\alpha-M O G$ antibodies to the pathogenesis of EAE in the SJL/J mouse strain seems to be more convincing. The EAE severity of WT SJL/L mice immunized with a low dose of PLP $_{139-151}$ could be increased by serum transfer and by direct administration of the anti-MOG monoclonal antibody 8.18C-5 [81]. Additionally, the housing of the TCR-transgenic mice under germ-free conditions results in a protection from spontaneous developed EAE, accompanied by low anti-MOG antibody titers. In turn, a recolonization of the gut reestablishes the spontaneous EAE susceptibility which is correlated with high anti-MOG antibody titers and a higher frequency of germinal center B cells within the lymph node [153].

\subsection{B cells as antigen-presenting cells}

B cells carry an antigen-specific B cell receptor enabling them to bind and process even small amounts of antigen and present it to $T$ cells. Therefore, their contribution to $E A E$ as antigen-presenting cells was investigated extensively. BM chimeric mice where MHC II deficiency is restricted to the B cell compartment, are resistant to rhMOG protein induction but not to MOG peptide or rmMOG protein induction, suggesting a role for $B$ cells as antigen-presenting cells after immunization with rhMOG. The resistance is accompanied by reduced frequencies of IFN $\gamma$ - and IL-17producing $\mathrm{CD}^{+}{ }^{+} \mathrm{T}$ cells in the periphery and a reduced number of CNS infiltrating $\mathrm{CD}^{+} \mathrm{T}$ cells. However, the ex vivo proliferation of splenocytes from either MHC II sufficient or MHC II deficient host animals is not affected [139]. The spontaneous double-transgenic models also reveal a role for $\mathrm{B}$ cells as antigen-presenting cells. MOG specific $T$ cells isolated from double-transgenic mice show a stronger proinflammatory cytokine production and proliferation when co-cultured with MOG specific $B$ cells instead of antigen-unspecific $B$ cells and recombinant rat MOG protein (rrMOG) [78, 79]. Nevertheless, conditional MHC-Il deletion in different APC subsets demonstrate that the antigen-presenting capacity of $B$ cells alone is not sufficient to trigger EAE [38]. It is also suggested that $B$ cells play a role in the initial 
steps of the reactivation phase within the CNS, leading to an increased proinflammatory milieu [154].

\subsection{B cells and cytokines}

The cytokine interleukin-6 (IL-6) plays a critical role in the development of EAE as IL6 deficient mice are completely resistant to MOG-peptide induced EAE [155]. B cells are the major source of IL-6 in secondary lymphoid tissues and abrogation of IL-6 producing $B$ cells via anti-CD20 treatment ameliorates EAE symptoms [56]. The depletion of IL- 6 producing B cells or the selective B cell IL-6 deficiency affects the frequency of $\mathrm{IL} 17^{+} \mathrm{CD} 4^{+} \mathrm{T}$ cells but had no effect on IFN $\gamma^{+} \mathrm{CD} 4^{+} \mathrm{T}$ cells $[56,139]$. Peripheral blood B cells from MS patients produce more IL-6 before Rituximab treatment and in vitro depletion of B cells from PBMCs of MS patients resulted in reduced IL-17 levels but had no effect on IFN $\gamma$ [139]. Additionally, ex vivo BCR and CD40-activated B cells isolated from PBMCs of RR-MS patients produced elevated levels of lymphotoxin and TNFa compared to B cells isolated from healthy controls, and the culture supernatant transfer triggered T cell proliferation in vitro [55]. B cells from the CNS of naïve C3HeB/Fej are the main producers of IL-12p35 and TNFa but their direct contribution to EAE development has not yet been investigated in detail [154].

\subsection{Regulatory B cells}

Besides the pro-inflammatory roles for $\mathrm{B}$ cells in the initiation and progression of $E A E$, it is also considered true that $B$ cells can act as regulatory cells exerting antiinflammatory functions. The regulatory B cell (Breg) subset was identified as CD19+CD1 ${ }^{\text {hi }}{ }^{+}$D $5^{+}$cells representing $1-2 \%$ of spleen $B 220^{+}$cells in WT mice [156]. BM chimera experiments reveal interleukin-10 (IL-10) as one of the crucial cytokine for Breg cells to exert their regulatory function [157]. Regulatory B cells can be expanded antigen-unspecific by CD40- and TLR stimulation in vitro [158]. Whereas TLR signaling alone triggers the production of IL-10, the simultaneous activation of CD40 and TLR4 leads to the production of IL-35 which in turn reduce the antigenpresenting potency of $B$ cells [159]. To exert their anti- inflammatory function in EAE, regulatory $B$ cells require $\mathrm{IL}-21$ and CD40 signaling, as well as cognate $\mathrm{T}$ cell interaction, shown by the fact that the transfer of IL-21 receptor knockout, CD40 knockout or MHC-II knockout regulatory B cells did not ameliorate EAE symptoms 
[160]. Upon EAE induction, there is an increase in the total number of IL10producing $B$ cells as well as a higher frequency of $C D 1 d^{\text {hi }} C D 5^{+}$cells among total CD19 $B$ cells [161]. Regulatory B cells influence $T$ cell effector functions directly, but they also act on other antigen-presenting cells [160, 161].

\subsubsection{Myeloid cells}

In addition to cells of the adaptive immune system, cells from the innate immune system are also critically involved in the development and progression of EAE and MS. Macrophage depletion studies in EAE models of mice and rats reveal beneficial effects for the disease [162-164]. The chemokine ligand for CCR2, macrophagechemoattractant protein-1 (MCP-1, CCL2), is upregulated in spinal cord tissue during EAE. Active immunization of MCP-1 knockout mice leads to an ameliorated disease course and MCP-1 knockout recipients who received MCP-1 sufficient T cells do not develop EAE $[165,166]$. In line with this, CCR2 knockout mice are relatively resistant to $E A E$ induction, whereas the transfer of CCR2-deficient T cells induce EAE like WT T cells $[165,167,168]$. On the cellular level, the EAE resistance is accompanied by a reduced number of CNS infiltrating mononuclear cells, especially macrophages, and no upregulation of inflammatory factors like CXCL10, CCL5 which caused the reduced $T$ cell attraction to the CNS. In contrast, neither absence of CCR2 nor of its ligand has an effect on $\mathrm{T}$ cell activation within the periphery. Bone-marrow chimera experiments identified the 'inflammatory' Ly6C ${ }^{\text {hi } C C R} 2^{+}$monocyte subset as the critical factor for EAE induction in CCR2 knockout animals [169].

These studies led to the conclusion that the solely presence of myelin-specific T cells is not sufficient for the development of an autoimmune disease, but the interaction of T cells with other cells types (like B cells or myeloid cells) is critically required for an autoimmune response.

\subsection{Objective}

There are several investigations studying the role of B cells in EAE and especially their impact on $T$ cells during the course of disease. However, their exact role and the 
critical time point when they contribute to the disease pathogenesis is not yet uncovered in detail.

In my PhD-thesis I resumed the investigations about T cell-B cell interaction in the MOG peptide active immunization EAE model. We have now developed a system where we are able to exclusively investigate the interplay between MOG-specific T cells and MOG-specific B cells in the different phases of EAE development. The main objectives of this study were:

1. To establish a model which makes it possible to track and analyze MOGspecific lymphocytes during the course of actively-induced EAE

2. To elaborate the critical time point and mechanism of the contribution of MOGspecific $B$ cells to the development and progression of EAE (activation phase, onset, recovery) 


\section{Material and Methods}

\subsection{Material}

\subsubsection{Reagents and Kits}

\section{Immunization}

Freund's Incomplete Adjuvant (IFA)

Difco Laboratories, US

Mycobacterium Tuberculosis H37 Ra

$\mathrm{MOG}_{\mathrm{p} 35-55}$

Difco Laboratories, US

Charité Berlin, Inst. for med.

Immunology

4-Hydroxy-3-nitrophenylacetyl

hapten-ovalbumin protein (NP-OVAL)

Biosearch Technologies, US

Ovalbumin

Sigma Aldrich, D

Pertussis toxin (PTX)

List Biological Laboratories, US

Ketamine

Medistar

Xylariem

Ecuphar

\section{ELISA}

BD OptEIA ${ }^{\mathrm{TM}}$ mouse IFN $\gamma$ ELISA Kit

BD, D

Mouse IL-17 ELISA reagents:

rec. mouse IL17A

mIL-17 biotinylated Det. Ab

mIL-17 MAb (Clone 50101) Cap. Ab

R\&D Systems, US

Mouse GM-CSF ELISA reagents:

rec. mouse GM-CSF

mGM-CSF biotinylated Det. Ab

mGM-CSF MAb

(Clone MP122E9) cap.Ab

R\&D Systems, US

Anti-mouse lgM Peroxidase

Sigma Aldrich, D

Anti-mouse lgG Peroxidase

Sigma Aldrich, D

3,3', 5,5' tetramethylbenzidine (TMB)

$B D, D$

\section{Cytometric Bead Array (CBA)}

Mouse Th1/Th2/Th17 Cytokine Kit

BD, D 


\section{Cell isolation kits}

EasySep $^{\mathrm{TM}}$ Mouse T Cell Enrichment Kit

EasySep $^{\mathrm{TM}}$ Mouse B Cell Isolation Kit

\section{RNA purification, cDNA synthesis, RT-PCR}

RNeasy Micro Kit

RNeasy Mini Kit

RevertAid First Strand cDNA Synthesis Kit

qPCR Master Mix

Primer and Probes

\section{Others}

Percoll

Trypan blue $(0.4 \%)$

Dextran 70000

$0.9 \% \mathrm{NaCl}$

Lymphocyte separation medium (LSM)

CFSE

Calibrite APC beads
Stemcell Technologies, CA

Stemcell Technologies, CA

Qiagen, D

Thermo Scientific, D

Eurogentec, D

Sigma Aldrich, D

GE Healthcare, D

Sigma Aldrich, D

Sigma Aldrich, D

Braun, D

Thermo Scientific, D

Thermo Scientific, D

BD,D

\subsubsection{Buffers and media}

If not otherwise stated, chemicals were purchased from Carl Roth, D.

\section{Standard buffers}

10x PBS:

$400 \mathrm{~g} \mathrm{NaCl}$

$10 \mathrm{~g} \mathrm{KCl}$

$71 \mathrm{~g} \mathrm{Na}_{2} \mathrm{HPO}_{4}$

$69 \mathrm{~g} \mathrm{NaH}_{2} \mathrm{PO}_{4}$, in $5 \mathrm{l}$ a.dest, $\mathrm{pH} 7.4$ 


\section{EH-medium (“Erhaltungsmedium"):}

13.38g/l Dulbeccos Modified Eagle Medium Powder (DMEM)

(Gibco, Life Technologies, D.)

$3.7 \mathrm{~g} / / \mathrm{NaHCO}_{3}$

25mM Hepes (Gibco, Life Technologies, D.) in a.dest

\section{Erythrocytes lysis buffer}

$0.15 \mathrm{M} \mathrm{NH}_{4} \mathrm{Cl}$

$1 \mathrm{mM} \mathrm{KHCO}_{3}$

0.1 mM EDTA, in a.dest $\mathrm{pH} 7.3$

FACS buffer: PBS, $2 \%$ BSA, $2 \% \mathrm{NaN}_{3}$

Sorting buffer: $\mathrm{EH}+2 \mathrm{mM}$ EDTA

Sorting collection buffer: $\mathrm{EH}+10 \%$ FCS

\section{rrMOG purification}

Sonification buffer: $\quad 2 x \mathrm{PBS} 300 \mathrm{mM} \mathrm{NaCl}, 25 \mathrm{mM} \mathrm{Na}_{2} \mathrm{HPO}_{4}, \mathrm{pH} 7.4$

Wash buffer: $\quad$ Sonification buffer+ $0.5 \% \mathrm{~N}, \mathrm{~N}$ Dimethyldodalylamin-N-oxid (LDAO)

Solubilisation buffer: $\quad 6 \mathrm{M}$ Guanidinium chloride $+10 \mathrm{mM}$ beta-mercaptoethanol

Column loading buffer: $\quad 1 \% \mathrm{NiCl}$ in a.dest

Column Washbuffer: $\quad 6 \mathrm{M}$ Guanidinium chloride+ $40 \mathrm{mM}$ imidazole

Elution buffer: $\quad 6 \mathrm{M}$ Guanidinium chloride+ $0.5 \mathrm{M}$ imidazole

Dialysis buffer 1: $\quad 1 \times$ PBS $+0.4 \mathrm{M}$ arginine $+50 \mathrm{mM}$ glutathione, $\mathrm{pH} 8$

Dialysis buffer 2: $\quad 1 \times \mathrm{PBS}+0.4 \mathrm{M}$ arginine, $\mathrm{pH} 8$

Inductor: $\quad 1 \mathrm{mM}$ IPTG (Thermo Scientific)

\section{$T$ and $B$ cell negative selection}

Isolation buffer : PBS, 5\% FCS, 2\% normal rat serum (provided by the manufacturer)

\section{Cell isolation from brain and spinal cord}

Resuspension/percoll dilution buffer: PBS, $1 \%$ Glucose, 0,1\% BSA 


\section{In vitro co-culture/ in vitro restimulation}

(cell culture supplements were purchased from Gibco, Life Technologies, D.)

Restimulation medium (ReMed)

RPMI 1640 pH 7.4

$1 \%$ non-essential amino acids

$1 \%$ sodium-pyruvate

$1 \%$ L-glutamine

$1 \%$ penicillin/streptamycine $(U)$

$5 \%$ FCS (GE Healthcare, D)

$0.2 \%$ B-MEtOH

\section{for ELISA}

coating buffer: $\quad$ IFN $\gamma$ : $\quad 0,1 \mathrm{M}$ Carbonate-Bicarbonate buffer

IL-17: $\quad$ phosphate buffer: $137 \mathrm{mM} \mathrm{NaCl}, 2.7 \mathrm{mM} \mathrm{KCl}, 8.1 \mathrm{mM}$ $\mathrm{Na}_{2} \mathrm{HPO}_{4}, 1.5 \mathrm{mM} \mathrm{NaH} \mathrm{PO}_{4}, \mathrm{pH} 7.2$

GM-CSF: PBS

wash buffer: $\quad$ PBS+ 0,05\% Tween20

blocking/dilution buffer: IFN $\gamma$ : PBS, 10\% FCS

IL-17: PBS, $1 \%$ BSA

GM-CSF: blocking: PBS, $5 \%$ sucrose, $1 \%$ BSA, $0.05 \%$ $\mathrm{NaN}_{3}$

dilution: TBS, 0,1\% BSA, 0,05\% Tween20

for serum antibody detection: $\quad$ PBS, 5\% BSA

\subsubsection{Equipment and consumables}

\subsubsection{Plastic ware}

Cell culture plates

Thermo Scientific, D

(6-well, 24-well, 96-well U-bottom/Flat-bottom)

Small reagent tubes $(0.2 \mathrm{ml}, 1,5 \mathrm{ml}, 2 \mathrm{ml})$

Sarstedt, D

Falcon tubes (15ml, 50ml)

Greiner Bio-One GmbH, D

$T$ and $B$ cell isolation tubes $(13 \mathrm{ml})$

Sarstedt, D

Tips ( $1 \mathrm{ml}, 0.2 \mathrm{ml}, 0.01 \mathrm{ml})$

StarLab, D

Filter Tips (1ml, $0.2 \mathrm{ml}, 0.01 \mathrm{ml})$

StarLab, D 
ELISA plate

Parafilm

Gloves

Combitips plus (5ml, 1ml, $0.5 \mathrm{ml}, 0.25 \mathrm{ml}$ )

Petri Dish

Syringes $(5 \mathrm{ml}, 10 \mathrm{ml})$

Insulin syringes $(1 \mathrm{ml})$

Sterile needles (24G, 20G, 27G, 26G)

FACS tubes (+/- Filter)

\subsubsection{Glas ware}

Glass pipettes (5ml, 10ml, 20ml)

Dounce tissue grinder +

Pestle (0.071-0.119mm) (0.02-0.056mm)

Neubauer cell chamber

Syringe for immunization (1ml, 2ml)

Tuberculin glass/ metal syringes

\subsubsection{Equipment}

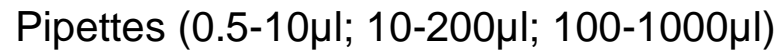

Pipettus

"Big Easy" silver EasySep magnet

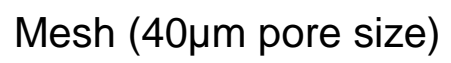

Multichannel pipette
Thermo Scientific Nunc, D

Picheney, US

Kimberly-Clark, US

Eppendorf, D

Greiner Bio-One GmbH, D

Braun, D

BD, D

Braun, D

BD, D

HBG, D

Kimble (Sigma-Aldrich, D)

Brand, D

Hartenstein, D

Eppendorf, D

Hirschmann, D

Stemcell Technologies, CA

UMG factory

StarLab, D 


\subsubsection{Machines}

RT-PCR Cycler

Flow Cytometer

Cell Sorter

Nanodrop

ELISA plate reader

Thermocycler

Centrifuges:

Multifuge $1 \mathrm{~S}-\mathrm{R}$

Centrifuge $5415 \mathrm{R}$

Incubator

Laminar flow

Inverted bright field

Microscope
StepOnePlus Real-Time PCR System (Applied Biosystems)

BD FACSCalibur

BD FACSAria II cell sorter

Peqlab, D

Tecan, $\mathrm{CH}$

Mastercycler (Eppendorf, D)

Heraeus, D

Eppendorf, D

Heraeus, D

Heraeus, D

Zeiss, D

\subsubsection{Software}

Microsoft Office

FlowJo V10

StepOnePlus Software v2.0

Tree Star, US

GraphPad Prism 5

Applied Biosystems

GraphPad Software, US

BD FACSDiva Software

BD CellQuestPro Software

PrimerExpress v2.0

Applied Biosystems

FCAP Array v3.0.1 Software for BD Cytometric Bead Array (CBA) Analysis 


\subsubsection{Flow cytometry fluorochrome labeled antibodies}

Table 1: Flow cytometry antibodies

\begin{tabular}{|c|c|c|c|}
\hline Specificity & Label & Clone & Company \\
\hline \multirow{2}{*}{ CD3e } & Alexa-Fluor 647 & $145-2 \mathrm{C} 11$ & BioLegend \\
\hline & Biotin & $145-2 C 11$ & $\mathrm{BD}$ \\
\hline \multirow{4}{*}{ CD4 } & APC & RM4-5 & BioLegend \\
\hline & APC-Cy7 & RM4-5 & BioLegend \\
\hline & $\mathrm{PE}$ & Gk1.5 & $\mathrm{BD}$ \\
\hline & PE-Cy5 & H129.19 & BioLegend \\
\hline \multirow{3}{*}{ CD8 } & PerCP & $53-6.7$ & $\mathrm{BD}$ \\
\hline & FITC & $53-6.7$ & $\mathrm{BD}$ \\
\hline & PE-Cy7 & $53-6.7$ & BioLegend \\
\hline \multirow{2}{*}{ CD25 } & APC & $3 \mathrm{C} /$ & BiolLegend \\
\hline & FITC & $3 \mathrm{C7}$ & BioLegend \\
\hline \multirow{2}{*}{ CD69 } & APC-Cy7 & $\mathrm{H} 1.2 \mathrm{~F} 3$ & BioLegend \\
\hline & FITC & $\mathrm{H} 1.2 \mathrm{~F} 3$ & BioLegend \\
\hline \multirow{2}{*}{ CD44 } & APC & IM7 & $\mathrm{BD}$ \\
\hline & APC-Cy7 & IM7 & BioLegend \\
\hline \multirow{2}{*}{ CD62L } & Biotin & MEL-14 & $\mathrm{BD}$ \\
\hline & FITC & MEL-14 & BioLegend \\
\hline \multirow{2}{*}{ CD45R/B220 } & APC & RA3-6B2 & BioLegend \\
\hline & PE & RA3-6B2 & $\mathrm{BD}$ \\
\hline MHC Class II $\left(I-A^{b}\right)$ & APC/PE/FITC & AF6-120.1 & BioLegend \\
\hline CD86 & FITC & GL-1 & BioLegend \\
\hline CD11a & Biotin & $2 \mathrm{D} 7$ & $\mathrm{BD}$ \\
\hline CD49d & Alexa-Fluor 647 & R1-2 & BioLegend \\
\hline $\mathrm{V} \beta 11 \mathrm{TCR}$ & FITC & KT11 & BioLegend \\
\hline $\mathrm{V} \beta 5.1 / 2 \mathrm{TCR}$ & Biotin & MR9-4 & $\mathrm{BD}$ \\
\hline CCR2 & Alexa Fluor 700 & 475301 & R\&D Systems \\
\hline $\mathrm{CX}_{3} \mathrm{CR}_{1}$ & APC & & \\
\hline Ly6C & APC-Cy7 & HK1.4 & BioLegend \\
\hline Gr-1 & $\mathrm{PE}$ & RB6-8C5 & $\mathrm{BD}$ \\
\hline \multirow{2}{*}{ CD11b } & APC & $\mathrm{M} 1 / 70$ & Biolegend \\
\hline & Biotin & M1/70 & BioLegend \\
\hline CD31 & APC & MEC13.3 & BioLegend \\
\hline Streptavidin- & $\begin{array}{c}\text { PE, APC, FITC, PE-Cy5, } \\
\text { APC-Cy7 }\end{array}$ & & Biolegend \\
\hline
\end{tabular}




\subsubsection{RT-PCR primer and probe sequences}

Table 2: RT-PCR Primer and probes (purchased from Sigma-Aldrich)

\begin{tabular}{|c|c|c|}
\hline \multirow[t]{3}{*}{ HPRT } & Fw (5`-3`) & TGCTCGAGATGTCATGAAGG \\
\hline & $\operatorname{Rev}\left(5^{\prime}-3^{\prime}\right)$ & TATGTCCCCCGTTGACTGAT \\
\hline & Probe & [6FAM]ATCACATTGTGGCCCTCTGT[TAM] \\
\hline \multirow[t]{3}{*}{ Integrin alpha $L$} & Fw (5`-3`) & AATGACGCTGGCAACAGATG \\
\hline & $\operatorname{Rev}\left(5^{\prime}-3^{\prime}\right)$ & GAGGTAAGTGTTCTGATCGCATGT \\
\hline & Probe & [6FAM]CTTTTGGCCTGTGACCCTGGACTGCT[TAM] \\
\hline \multirow[t]{3}{*}{ Integrin alpha 4} & Fw (5`-3`) & CGAGTTTCAAGCAGTGGAGAGA \\
\hline & $\operatorname{Rev}\left(5^{\prime}-3^{\prime}\right)$ & TGGTATGTGGCCTCTACATGAATG \\
\hline & Probe & [6FAM]CACACCAGGCATTCATGCGGAAAGAC[TAM] \\
\hline \multirow[t]{3}{*}{ IFNy } & Fw (5`-3`) & TCAAGTGGCATAGATGTGGAAGAA \\
\hline & $\operatorname{Rev}\left(5^{\prime}-3^{\prime}\right)$ & TGGCTCTGCAGGATTTTCATG \\
\hline & Probe & [6FAM]TCACCATCCTTTTGCCAGTTCCTCCAG [TAM] \\
\hline \multirow[t]{3}{*}{ GM-CSF } & Fw (5`-3`) & GGGCGCCTTGAACATGAC \\
\hline & $\operatorname{Rev}\left(5^{\prime}-3^{\prime}\right)$ & CGCATAGGTGGTAACTTGTGTTTC \\
\hline & Probe & [6FAM]CCCCCCAACTCCGGAAACGGA [TAM] \\
\hline \multirow[t]{3}{*}{ IL-17A } & Fw (5`-3`) & ACTTTCAGGGTCGAGAAGATGCT \\
\hline & $\operatorname{Rev}\left(5^{\prime}-3^{\prime}\right)$ & TTCTGAATCTGCCTCTGAATCCAC \\
\hline & Probe & [6FAM]TGGGTGTGGGCTGCACCTGC [TAM] \\
\hline \multirow[t]{3}{*}{ ALCAM } & Fw (5`-3’) & ACGCGACTGTGGTGTGGAT \\
\hline & $\operatorname{Rev}\left(5^{\prime}-3^{\prime}\right)$ & CCTGATAATGAAGACTGGAAAAGGA \\
\hline & Probe & [6FAM]AAGGATAACATCCGGCTCCGGTCCA [TAM] \\
\hline \multirow[t]{3}{*}{ ICAM } & Fw (5`-3`) & GCCAAGCCCACGCTACCT \\
\hline & $\operatorname{Rev}\left(5^{\prime}-3^{\prime}\right)$ & TCTCTGGGATGGATGGATACCT \\
\hline & Probe & [6FAM]TCACCGTTGTGATCCCTGGGCCT [TAM] \\
\hline \multirow[t]{3}{*}{ VCAM } & Fw (5`-3`) & CAGAGTGTACAGCCTCTTTATGTCAAC \\
\hline & $\operatorname{Rev}\left(5^{\prime}-3^{\prime}\right)$ & GGACTGCCCTCCTCTAGTATAGGA \\
\hline & Probe & [6FAM]TTGCCCCCAAGGAAACCACCATC [TAM] \\
\hline \multirow[t]{3}{*}{ CCR7 } & Fw (5`-3`) & CAGCCTTCCTGTGTGATTTCTACA \\
\hline & $\left.\operatorname{Rev}\left(5^{\prime}-3\right)^{\prime}\right)$ & ACCACCAGCACGTTTTTCCT \\
\hline & Probe & [6FAM]CAGAGCACCATGGACCCAGGGAAAC [TAM] \\
\hline \multirow[t]{3}{*}{ CXCR3 } & Fw (5`-3`) & CCAAGCCATGTACCTTGAGGTTAG \\
\hline & $\left.\operatorname{Rev}\left(5^{\prime}-3\right)^{\prime}\right)$ & AATCGTAGGGAGAGGTGCTGTTT \\
\hline & Probe & [6FAM]ATGCCTCGGACTTTGCCTTTCTTCTGG [TAM] \\
\hline \multirow[t]{3}{*}{ CXCR4 } & Fw (5`-3`) & ACCTCTACAGCAGCGTTCTCATC \\
\hline & $\operatorname{Rev}\left(5^{\prime}-3^{\prime}\right)$ & TGTTGGTGGCGTGGACAATA \\
\hline & Probe & [6FAM]TGGCCTTCATCAGCCTGGACCG [TAM] \\
\hline \multirow[t]{3}{*}{ CCR5 } & Fw (5`-3`) & TGCTCAACCTGGCCATCTCT \\
\hline & $\operatorname{Rev}\left(5^{\prime}-3^{\prime}\right)$ & CCCACTCATTTGCAGCATAGTG \\
\hline & Probe & [6FAM]CTGCTCTTCCTGCTCACACTACCATTCTGG [TAM] \\
\hline \multirow[t]{3}{*}{ CCR6 } & Fw (5`-3`) & TCGTCCAGGCAACCAAATC \\
\hline & $\left.\operatorname{Rev}\left(5^{\prime}-3\right)^{\prime}\right)$ & CCACACTGCCACACAGATGAC \\
\hline & Probe & [6FAM]TTCCGGGTACGCTCCAGAACACTGA [TAM] \\
\hline \multirow[t]{3}{*}{ CCL5 } & Fw (5’-3’) & GGAGTATTTCTACACCAGCAGCAA \\
\hline & $\left.\operatorname{Rev}\left(5^{\prime}-3\right)^{\prime}\right)$ & CACACACTTGGCGGTTCCTT \\
\hline & Probe & [6FAM]TGCTCCAATCTTGCAGTCGTGTTTGTCA [TAM] \\
\hline \multirow[t]{3}{*}{ CXCL10 } & Fw (5`-3`) & CATCCCTGCGAGCCTATCC \\
\hline & $\operatorname{Rev}\left(5{ }^{\prime} 3^{\prime}\right)$ & CATCTCTGCTCATCATTCTTTTTCA \\
\hline & Probe & [6FAM]CCCACGTGTTGAGATCATTGCCACG [TAM] \\
\hline CXCL12 & Fw (5’-3`) & CAAGCATCTGAAAATCCTCAACAC \\
\hline
\end{tabular}




\begin{tabular}{|l|l|l|}
\hline & Rev (5`-3`) & GCACACTTGTCTGTTGTTGTTCTTC \\
\cline { 2 - 3 } & Probe & [6FAM]AAACTGTGCCCTTCAGATTGTTGCACG [TAM] \\
\hline
\end{tabular}

\subsection{Methods}

\subsubsection{Mouse strains}

All mice strains were bred in IVC cages (SPF conditions) in the central animal facility of the University Medicine School Göttingen. Experiments were performed with at least 10 weeks old mice. All mice were on the C57bl/6 background. Animal experiments were approved by the responsible authorities in Lower Saxonia.

Table 3: Used mouse strains

\begin{tabular}{|c|c|c|}
\hline Name & used for & \begin{tabular}{|l} 
Ref. \\
\end{tabular} \\
\hline C57bl/6 & Host & \\
\hline RFP & Crossed to various mouse strains & {$[170$} \\
\hline GFP & Crossed to various mouse strains & {$[171$} \\
\hline 2D2 & Isolation of MOG-specific T cells & [77] \\
\hline 2D2RFP & Isolation of RFP ${ }^{+}$MOG-specific T cells & \\
\hline 2D2GFP & Isolation of GFP ${ }^{+}$MOG-specific T cells & \\
\hline Th & Isolation of MOG-specific B cells & {$[143$} \\
\hline ThRFP & Isolation of RFP ${ }^{+}$MOG-specific B cells & \\
\hline THGFP & Isolation of $\mathrm{GFP}^{+}$MOG-specific B cells & \\
\hline B1.8 (NP) & Isolation of NP-specific B cells & {$[172$} \\
\hline OT-II & Isolation of OVA-specific T cells, used as host & {$[173$} \\
\hline OT-II x NP & Host & \\
\hline mb1Cre & Crossed to XBP- $1^{\mathrm{t} / \mathrm{fl}}$ & {$[174$} \\
\hline XBP-1 $1^{\mathrm{t} / \mathrm{t} / \mathrm{T}}$ & & {$[175$} \\
\hline MHC-II- & $\begin{array}{l}\text { Crossed to Th mice for isolation of MHC- }-\mathrm{II}^{-1-} \text { MOG- } \\
\text { specific B cells }\end{array}$ & {$[176$} \\
\hline FOXP3-eGFP & $\begin{array}{l}\text { Crossed to 2D2RFP for isolation of MOG-specific } \\
\text { FOXP3 eGFP reporter T cells }\end{array}$ & {$[177]$} \\
\hline
\end{tabular}




\subsubsection{Antigen-specific $T$ and $B$ cell isolation}

Briefly, lymph nodes and spleens were taken out from the respective donor animals (Table 3) and single cell suspension was prepared by smashing the organs through a mesh $\left(40 \mu \mathrm{m}\right.$ pore size). After centrifugation (1200rpm, 8 min., $\left.4^{\circ} \mathrm{C}\right)$ the cells were resuspended at a concentration of $1 \times 10^{8}$ cells $/ \mathrm{ml}$ in isolation buffer. $\mathrm{CD}^{+} \mathrm{T}$ cells and $\mathrm{CD}{ }^{+} \mathrm{B}$ cells were isolated using the EasySep Mouse T Cell Enrichment Kit (Stemcell) or the EasySep Mouse B Cell Isolation Kit, respectively. Cell isolation was performed following the manufacturer's protocol for the "Big Easy" silver EasySep magnet. Cell purity was routinely determined by flow cytometry and was always higher than $90 \%$. After purification, the cells were used for various in vitro or in vivo experiments.

\subsubsection{Adoptive transfer experiments}

For adoptive cell transfer the cells were injected intravenously (i.v.) in different cell numbers depending on the experiment in a total volume of $200 \mu$ I PBS into different host mice. For C57BL/6 experiments $7-8 \times 10^{6} \mathrm{~T}$ cells were injected either alone or together with $1 \times 10^{7}$ MOG-specific B cells if not otherwise stated. For OT-II or OT-II $x$ NP experiments, $3.5 \times 10^{6} \mathrm{MOG}$-specific T cells were either injected alone or together with $1 \times 10^{7}$ MOG-specific $B$ cells. Two days post adoptive cell transfer, the host mice were immunized with the respective antigen emulsified in CFA like described in chapter 2.2.5.2.

\subsubsection{CFSE-labeling}

To assess $T$ cell proliferation in vitro or ex vivo, isolated antigen-specific $T$ cells were labeled with carboxyfluorescein diacetate succinimidyl ester (CFSE). Briefly, the respective lymphocytes were incubated at a concentration of $2 \times 10^{6}$ cells $/ \mathrm{ml}$ PBS containing $0.25 \mu \mathrm{M}$ CFSE for $10 \mathrm{~min}$ at $37^{\circ} \mathrm{C}$. The reaction was stopped by addition of $10 \mathrm{ml} \mathrm{FCS}$ and the cells were washed twice in PBS containing 5\% FCS. According to the experiment, the CFSE labeled cells were either injected i.v. into host animals to determine the proliferation ex vivo at different time points or cultured with different conditions (chapter 2.2.7.1.) for three days. The proliferation, indicated by the CFSE dilution with every cell division, was assessed by flow cytometry. 


\subsubsection{EAE induction}

\subsubsection{Antigens}

The 2D2 TCR epitope $M_{0} G_{p 35-55}$ (MEVGWYRSPFSRVVHLYRNGK) was purchased from Charité, Institute for Medical Immunology. 4-Hydroxy-3-nitrophenylacetyl hapten conjugated to OVA (ovalbumin) protein (NP-OVAL) was purchased from Biosearch Technologies. A plasmid construct encoding the extracellular domain of rat MOG protein (MOG amino acids 1-125) was provided by C. Linington (University of Glasgow, UK) and purified from bacterial inclusion bodies [178]. Expression plasmidPQE-12 containing rat MOG 1-125 was grown in LB medium containing ampicillin $(100 \mathrm{mg} / \mathrm{ml})$ and kanamycin $(25 \mathrm{mg} / \mathrm{ml})$. MOG expression was induced with isopropyl thiogalactoside (IPTG) before the cells were pelletted. The pellet was resuspended in sonibuffer with lysozyme, sonicated and mechanically disrupted and homogenized (all used buffers for rrMOG purification are listed in chapter 2.1.2.). Lysed samples were washed, resuspended in solubilization buffer, and loaded onto the Ni-NTA column prepared with chelating sepharose and $1 \% \mathrm{NiCl}_{2}$. The column was washed with column washbuffer and the protein was eluted with column elution buffer. The MOG protein was refolded in several dialysis steps. For the first $24-48 \mathrm{~h}$ it was dialyzed against dialysis buffer 1 followed by a dialysis against dialysis buffer 2 ON. Final dialysis was performed against $1 \times$ PBS for $48 \mathrm{~h}$. The protein concentration was measured on a Nanodrop at $280 \mathrm{~nm}$.

\subsubsection{Immunization and scoring}

Two days post adoptive cell transfer (chapter 2.2.3.) EAE was induced by immunization with respective antigen in at least 10 weeks old mice.

Mice were anesthetized with $100 \mu \mathrm{l}$ of $10 \%$ ketamine, $5 \%$ xylariem in $0,9 \% \mathrm{NaCl}$ per

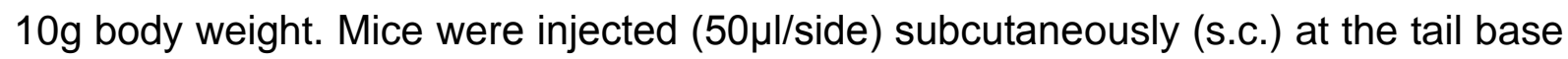


$\mathrm{MOG}_{\mathrm{p} 35-55}$ in PBS. CFA was prepared by addition of $10 \mathrm{mg}$ mycobacterium tuberculosis (strain $\mathrm{H} 37 \mathrm{Ra}$ ) to $10 \mathrm{ml}$ incomplete freund's adjuvant $(1 \mathrm{mg} / \mathrm{ml})$. Pertussis toxin (PTX) (200ng/day) was injected intraperitoneal (i.p.) on days 0 and 2. Concentrations of other antigens were different in each experiment and are stated in the figure legend. 
Animals were scored daily for clinical signs of EAE, based on a scoring system reaching from $0-10$ points with the individual points defined as follows:

$0=$ healthy

$1=$ reduced tail tonicity

2 = flaccid tail paralysis

$3=$ loss of righting reflex

$4=$ kinetic ataxia

$5=$ slight paralysis of hind legs

$6=$ plegia of one leg or moderate paralysis of both hind legs

$7=$ paraplegia with complete paralysis of both hind legs

$8=$ tetraparesis with additional (slight) paralysis of both forelegs

$9=$ moribund

$10=$ dead $\quad$ With a score of $>7$ the animals had to be sacrifice.

\subsubsection{Tissue preparation for cell isolation}

\subsubsection{Lymphocyte isolation from lymph nodes and spleen}

To analyze the injected antigen-specific $T$ and $B$ cells ex vivo, lymph nodes (inguinal and paraaortic as draining lymph nodes, axilliary and cervical lymph nodes as nondraining lymph nodes) and spleen were isolated from the respective host mice and single cell suspension was prepared like described above (chapter 2.2.2.). Due to expression of fluorescent markers (either RFP or GFP) it was possible to track the cells with flow cytometry (chapter 2.2.11.) or cell sorting (chapter 2.2.12.) after recovery.

\subsubsection{Lymphocyte isolation from peripheral blood}

If animals were euthanized, blood was taken by heart puncture and mixed in a 1:2 ratio with $2 \mathrm{mM}$ ethylenediaminetetraacetic acid (EDTA) in PBS to avoid thrombocyte aggregation. In case animals were kept alive, blood was taken by retro-orbital puncture. Lymphocytes were separated via density gradient centrifugation using lymphocyte separation medium (LSM) at $1500 \mathrm{rpm}, 20 \mathrm{~min}, 4^{\circ} \mathrm{C}$. The interphase between plasma and separation solution containing the lymphocytes was transferred into a new tube and washed with PBS. 


\subsubsection{Lymphocyte isolation from spinal cord and brain}

Euthanized mice were perfused through the left cardiac ventricle with cold 0,9\% $\mathrm{NaCl}$. The spinal cord and brain were extracted and kept in PBS. Single-cell suspensions were prepared by application of mechanical disruption and subsequent resuspension in spinal cord resuspension buffer. After centrifugation, the pellet was resuspended in $30 \%$ Percoll solution and mononuclear cells were separated from myelin via Percoll density gradient centrifugation (70/45/30\% Percoll in spinal cord resuspension buffer)(2300rpm, $20 \mathrm{~min}, 4^{\circ} \mathrm{C}$ w/o break). The interphases (30:45 \% and $45: 70 \%$ ) containing the lymphocytes were transferred into a new tube and washed with PBS.

For isolation of perivascular lymphocytes, spinal cord tissue was prepared following the spinal cord microvessel endothelial cell isolation protocol and T cells were sorted according to their $\mathrm{RFP}^{+}$expression.

\subsubsection{Isolation of spinal cord microvessel endothelial cells}

The spinal cord was extracted and kept in EH medium. To prepare a cell suspension, the spinal cord was cut in small pieces $\left(1-5 \mathrm{~mm}^{2}\right)$ and homogenized in a $7 \mathrm{ml}$ Dounce tissue grinder (Kimble/Kontes), first with a larger clearance pestle $(0.071-0,119 \mathrm{~mm})$ and then with a smaller clearance pestle $(0,02-0,056 \mathrm{~mm})$. The homogenate was then spun down (1200rpm, $8 \mathrm{~min}, 4^{\circ} \mathrm{C}$ ) and resuspended in $6 \mathrm{ml}$ of an $18 \%(\mathrm{w} / \mathrm{v})$ dextran solution (MW 70000) in $\mathrm{EH}$ medium. To get rid of the myelin proteins, the resuspension was spun down at $10000 \mathrm{xg}$ for $20 \mathrm{~min}$ and the supernatant was removed. The pellet was resuspended in EH medium and washed (1200rpm, $8 \mathrm{~min}$, $4^{\circ} \mathrm{C}$ ). To digest the microvessels, the pellet was resuspend in $600 \mu 11 \%$ collagenase and gently shaked at $37^{\circ} \mathrm{C}$ for $20 \mathrm{~min}$. Afterwards, the suspension was transferred into a FACS tube, washed with FACS buffer and stained for CD31 for CD $31^{+}$cell sorting. 


\subsubsection{In vitro experiments}

\subsubsection{In vitro co-culture experiments}

To study the interaction of antigen-specific $T$ and $B$ cells in vitro, respective $T$ and $B$ cells were isolated as described in chapter 2.2.2. in sterile conditions. $1 \times 10^{6} \mathrm{~T}$ cells and $2 \times 10^{6} \mathrm{~B}$ cells were co-cultured for 3 days with increasing concentrations of different antigens in a range from $0-10 \mu \mathrm{g} / \mathrm{ml}$ in a 24-well plate in ReMed in an incubator at $37^{\circ} \mathrm{C}$ and $5 \% \mathrm{CO}_{2}$. To determine soluble factors in the supernatant, it was removed 3 days post culture and stored in $-80^{\circ} \mathrm{C}$ until analysis. Depending on the experiment, either the $T$ cells or the B cells were CFSE labeled (chapter 2.2.4.) before co-culture and proliferation was analyzed 3 days post culture.

\subsubsection{In vitro restimulation experiments}

To investigate the cytokine production of transferred T cells ex vivo, single cell suspension was prepared from draining lymph nodes and spleen. Erythrocytes were lysed from splenocytes by adding $1 \mathrm{ml}$ erythrocyte lysis buffer for $5 \mathrm{~min}$ on ice. Afterwards, $5 \times 10^{5}$ total lymph node cells and splenocytes were cultured with increasing concentrations of $\mathrm{MOG}_{\mathrm{p} 35-55}(0,1,5,10 \mu \mathrm{g} / \mathrm{ml})$ in $100 \mu \mathrm{l}$ ReMed in a 96well plate for $72 \mathrm{~h}$ (incubator, $37^{\circ} \mathrm{C}, 5 \% \mathrm{CO}_{2}$ ). The cell culture supernatant was collected and stored at $-80^{\circ} \mathrm{C}$ until cytokine determination via ELISA.

\subsubsection{Cell count}

Total cell numbers from single cell suspensions were determined using a Neubauer cell chamber. Trypan blue containing $1 \%$ acidic acid for erylysis was used to distinguish between viable and non-viable cells.

cell number: $\mathrm{N} x$ dilution factor $\mathrm{x}$ volume $\times 10^{4} \quad \mathrm{~N}=$ number of living cells

Cell number of adoptively transferred $\mathrm{RFP}^{+}$or $\mathrm{GFP}^{+}$cells was determined with $\mathrm{BD}$ Calibrite APC beads by flow cytometry. A defined number of beads was solved in PBS and $50 \mu \mathrm{l}$ of the suspension was added to $200 \mu \mathrm{l}$ of an organ single cell suspension with defined volume. Weight of respective organs (lymph nodes, spleen and spinal cord) was determined before smashing to enable calculation of cell number per gram. 
Total cell number was calculated according to the equation:

$$
\left(\frac{\text { beads in } 50 \mu l x \text { acquired cells }}{\text { acquired beads }}\right) \times \text { dilution factor }
$$

To determine the cell number per gram organ weight, the total number had to be divided by organ weight $(\mathrm{g})$.

\subsubsection{Enzyme linked immunosorbent assay (ELISA)}

\subsubsection{Cytokine ELISA}

The amounts of IFN $\gamma$, IL-17A and GM-CSF were determined from supernatants of restimulated total draining lymph node cells and splenocytes (chapter 2.2.7.2.) using BD OptEIA mouse ELISA Kits for IFN $\gamma$ or capture antibody, detection antibody and standard from R\&D Systems for IL-17A and GM-CSF following the manufacturer's protocol. ELISA plates were coated with $50 \mu \mathrm{l}$ of respective capture antibody diluted 1:250 in ELISA coating buffer $\mathrm{ON}$ at $4^{\circ} \mathrm{C}$. For quantification, supernatant samples were diluted (IFN $\gamma$ 1:50; IL-17A 1:5; GM-CSF 1:20) and analyzed in triplicates. For HRP enzymatic reaction $100 \mu l$ 3,3', 5,5' tetramethylbenzidine (TMB) was added for $30 \mathrm{~min}$ and the reaction was stopped by addition of $50 \mu \mathrm{l} 1 \mathrm{M} \mathrm{H}_{2} \mathrm{SO}_{4}$. The absorbance was measured at $450 \mathrm{~nm}$ with correction wavelength of $620 \mathrm{~nm}$ in an ELISA reader.

\subsubsection{Serum antibody ELISA}

For determination of $\alpha-M O G \operatorname{IgM}$ or $\lg G$ antibodies, ELISA plates were either coated with $4 \mu \mathrm{g} / \mathrm{ml} \mathrm{rrMOG}$ or $10 \mu \mathrm{g} / \mathrm{ml} \mathrm{MOG}_{\mathrm{p} 35-55}$ in PBS ON at $4{ }^{\circ} \mathrm{C}$. For determination of $\alpha$ NP IgM or IgG antibodies, plates were coated with $5 \mu \mathrm{g} / \mathrm{ml} \mathrm{NP-OVAL} \mathrm{or} 5 \mu \mathrm{g} / \mathrm{ml}$ OVA as control. To determine antibody titers, sera samples were diluted with dilution buffer 1:100 and 1:1000 and analyzed in triplicates. HRP-conjugated detection antibodies against mouse IgM and IgG were used in a 1:250 dilution. Further analysis steps were performed as described above.

\subsubsection{Cytometric Bead Array (CBA)}

With the Mouse Th1/Th2/Th17 Cytokine Kit the cytokines IL-2, IL-4, IL-6, IFN- $\gamma$, TNFa, IL-17A, IL-10 were assessed in the supernatants of in vitro co-culture assays. The 
supernatants of different $\mathrm{T}$ cell-B cell in vitro co-culture experiments were collected after $72 \mathrm{~h}$ and stored in $-80^{\circ} \mathrm{C}$ until cytokine quantification. Before analysis, supernatant samples were diluted 1:10 with dilution buffer (provided from the Kit).

$10 \mu$ of each supernatant dilution was mixed with $10 \mu$ l Capture Beads (beads with different fluorescence intensities linked to antibodies against the 7 different cytokines) and $10 \mu \mathrm{PE}$ - detection solution (PE-conjugated antibodies). Furthermore, a dilution series from a standard (from kit, consisting of cytokines) was provided and also mixed with capture beads and PE- detection solution. After an incubation time of $2 \mathrm{~h}$ at RT in the dark, the samples were washed once in washing buffer (from kit), resuspend in $50 \mu \mathrm{l}$ washing buffer and analyzed with flow cytometry. Cytokine concentrations were determined with the help of the standard curve and the FCAP Array v3.0.1 Software.

\subsubsection{Flow cytometry}

$1-5 \times 10^{6}$ cells per sample was surface stained in $100 \mu \mathrm{F}$ FCS buffer with combinations of monoclonal antibodies labelled with fluorochromes for $15 \mathrm{~min}$ at $4^{\circ} \mathrm{C}$. After staining, the samples were washed and resuspended in FACS buffer. Stained cells were either analyzed on a BD FACSCalibur or on a BD FACSAria II cell sorter. Final analysis was performed using FlowJo V10 (Tree Star) software. The injected antigen-specific $T$ and $B$ cells could be tracked due to their expression of either RFP or GFP. All used antibodies are listed in Table1.

\subsubsection{Cell sorting}

Cell sorting was performed with the BD FACSAria II cell sorter. For RNA isolation, cDNA synthesis and subsequent RT-PCR, ex vivo isolated MOG-specific CD4 ${ }^{+} \mathrm{T}$ cells from different organs (chapter 2.2.6.) were identified and sorted by their simultaneous expression of either GFP or RFP and CD4. The sorted cells were collected in EH medium containing 10\% FCS and subsequently resuspended in RLT buffer (chapter 2.2.13.) for RNA extraction. Endothelial cells from spinal cord microvessels were sorted according to their CD31 expression.

\subsubsection{RNA isolation, CDNA synthesis and RT-PCR}

RNA isolation was performed using Qiagen RNeasy Micro Kit for $<100000$ cells or Qiagen RNeasy Mini Kit $>100000$ cells following the manufacturer's protocol. As the total RNA amount from less than 100.000 cells was very low, the RNA concentration 
could not be determined and whole eluat was used for subsequent CDNA synthesis. cDNA synthesis was performed using RevertAid First Strand cDNA Synthesis Kit with random hexamer primer as described in the manufacturer's protocol. RT-PCR was performed with the GPCR Master Mix on a StepOnePlus Real-Time PCR System. RT-PCR was performed with target specific FAM and TAMRA labeled TaqMan probes. Analysis was performed using StepOnePlus Software v2.0. Expression was normalized to that of the housekeeping gene hypoxanthine phosphoribosyltransferase 1 (HPRT). The best working forward and reverse primer concentration was titrated and used primer and probe sequences are listed in Table 2.

\subsubsection{Statistics}

Statistical analysis was performed with GraphPad Prism 5. Data are depicted as mean \pm SEM (standard error of the mean). Respective statistical analysis methods are always depicted in the figure legend. P-value of statistical difference was indicated like: ns: $p \geq 0.05 ;{ }^{*}: p \leq 0.05 ;{ }^{* *}: p \leq 0.01 ;{ }^{* \star *}: p \leq 0.001$ 


\section{Results}

\subsection{Establishment of the EAE model}

Several publications suggest an interaction of $T$ and $B$ cells to be involved in the pathogenesis of EAE. Most of the studies make use of the C57bl/6 active immunization with $M O G$ antigens $\left(M_{0} G_{p 35-55}\right.$ (MOG peptide), rhMOG (recombinant human MOG), rrMOG (recombinant rodent MOG)) EAE model where the amplification of respective myelin-specific $T$ and $B$ cells is triggered. However, by their investigation of the endogenous $T$ and $B$ cell pool they do not exclusively focus on myelin-specific lymphocytes. We now developed a system enabling us to track and analyze MOG-specific T and B cells during the whole course of EAE ex vivo.

As the antigen-specificity of pathogenic lymphocytes is critically associated with the development of EAE, it is advantageous to generate a system which facilitates the tracking of antigen-specific $T$ and $B$ cells during the course of EAE. For this purpose, we isolated MOG-specific T cells from 2D2 mice [77] and MOG-specific B cells from Th mice [143]. Beside the transgenic TCR or BCR, these cells simultaneously express either red fluorescent protein (RFP) or green fluorescent protein (GFP), enabling the cell tracking during the whole course of disease and to ex vivo analyze them with the help of different techniques (flow cytometry, FACS sorting, 2-photon intravital imaging). To study the cellular behavior during the course of EAE, we transferred the cells intravenously (i.v.) into different host animals followed by active immunization with different antigens. Initial experiments were performed with $\mathrm{C} 57 \mathrm{bl} / 6$ (WT) recipient mice. To study the influence of MOG-specific B cells on MOG-specific $T$ cells in detail we utilized OT-II mice as recipients. These mice harbor $>90 \%$ of endogenous OVA-specific $T$ cells allowing to minimize the endogenous $T$ cell responses to MOG antigens. To investigate the effect of different genetically modified $B$ cells on EAE, we utilized OT-II $\times$ NP as these mice are devoid of endogenous pathogenic $T$ cells and $B$ cells.

To evaluate the activation status of freshly isolated MOG-specific $T$ cells, we performed antibody-labeled surface staining for flow cytometry analysis before i.v. transfer. To ensure that the transferred MOG-specific $T$ cells can be primed antigenspecific in vivo, we analyzed their proliferation behavior ex vivo upon $\mathrm{MOG}_{\mathrm{p} 35-55}$ or OVA protein immunization. 


\subsubsection{Freshly isolated MOG-specific $T$ cells have a naïve phenotype and exhibit an antigen-specific response in vivo}

To initially characterize MOG-specific $T$ cells before transfer, they were surface stained with antibodies against CD3, CD4, CD8 (Fig. 4 A) and the surface markers CD62L, CD69 and CD44 (Fig. 4 B) followed by flow cytometry analysis. CD62L is highly expressed by naïve T cells, whereas CD69 is considered as an early activation marker. CD44 is mainly expressed by antigen-experienced T cells. For lymphocyte isolation, lymph nodes and spleens from the respective donor mice were prepared and cell isolation was carried out using a negative selection kit to avoid TCR or BCR cross-linking and cell activation.
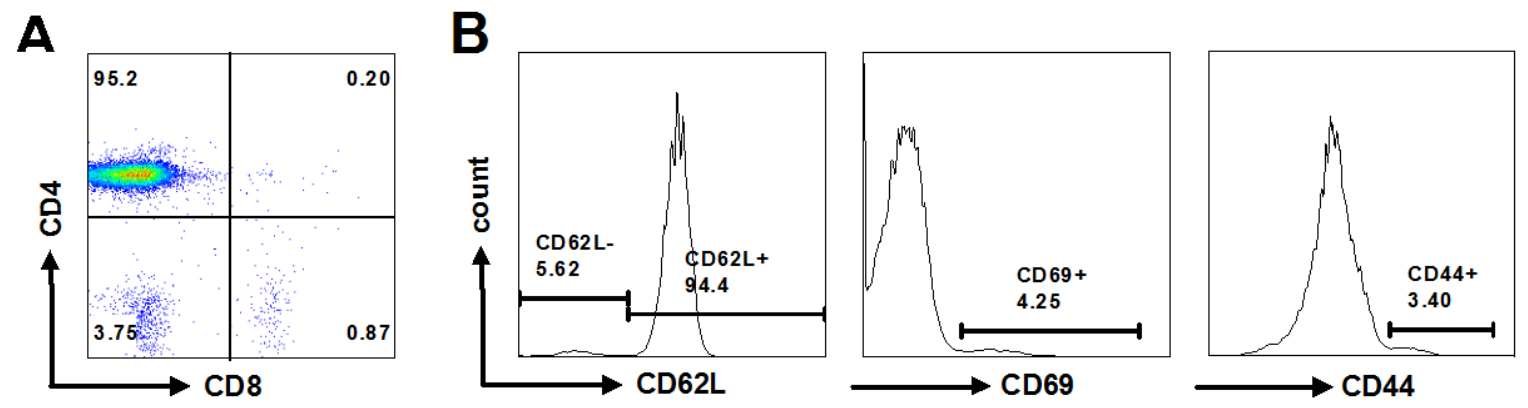

Figure 4: Freshly isolated MOG-specific $T$ cells from 2D2 mice have a naïve phenotype. MOG-specific $T$ cells were isolated from 2D2 mice and antibody-labelled against (A) CD4 and CD8 (gated on CD3) and (B) CD62L, CD69 and CD44 (gated on CD4) for flow cytometry analysis. Histogram gates depict the percentage of surface marker positive cells.

Approximately $95 \%$ of the isolated $\mathrm{CD}^{+} \mathrm{T}$ cells were $\mathrm{CD}^{+}$(Fig. $\left.4 \mathrm{~A}\right)$. The majority (94\%) of the $\mathrm{CD}^{+}{ }^{+} \mathrm{T}$ cells were $\mathrm{CD} 2 \mathrm{~L}^{+}$and only a minor proportion of the isolated $\mathrm{T}$ cells expressed the activation marker CD69 $(4.25 \%)$ or the memory marker CD44 (3.39\%) (Fig. 4 B) assuming that the vast majority of the transferred MOG-specific T cells had a naïve phenotype.

To proof whether the transferred MOG-specific $T$ cells respond specifically to the MOG peptide antigen in vivo, freshly isolated RFP ${ }^{+}$MOG-specific $\mathrm{T}$ cells were labeled with CFSE and injected i.v. into C57bl/6 WT mice. Two days later, the host

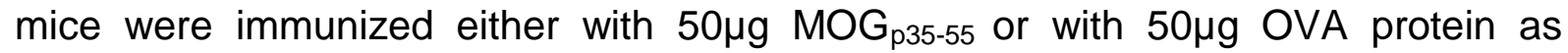
control. CFSE is a membrane-permeable fluorescent dye, which is non-fluorescent in its native form. Due to enzymatic modification within the cell, it is rendered highly fluorescent and also unable to diffuse out of it. Upon one cycle of cell division the 
stain is halved in each of the daughter cells, which can be detected by flow cytometry. Five days post immunization (d p.i.), single cell suspension from spleen and draining lymph node (inguinal) was analyzed for CFSE dilution by flow cytometry.

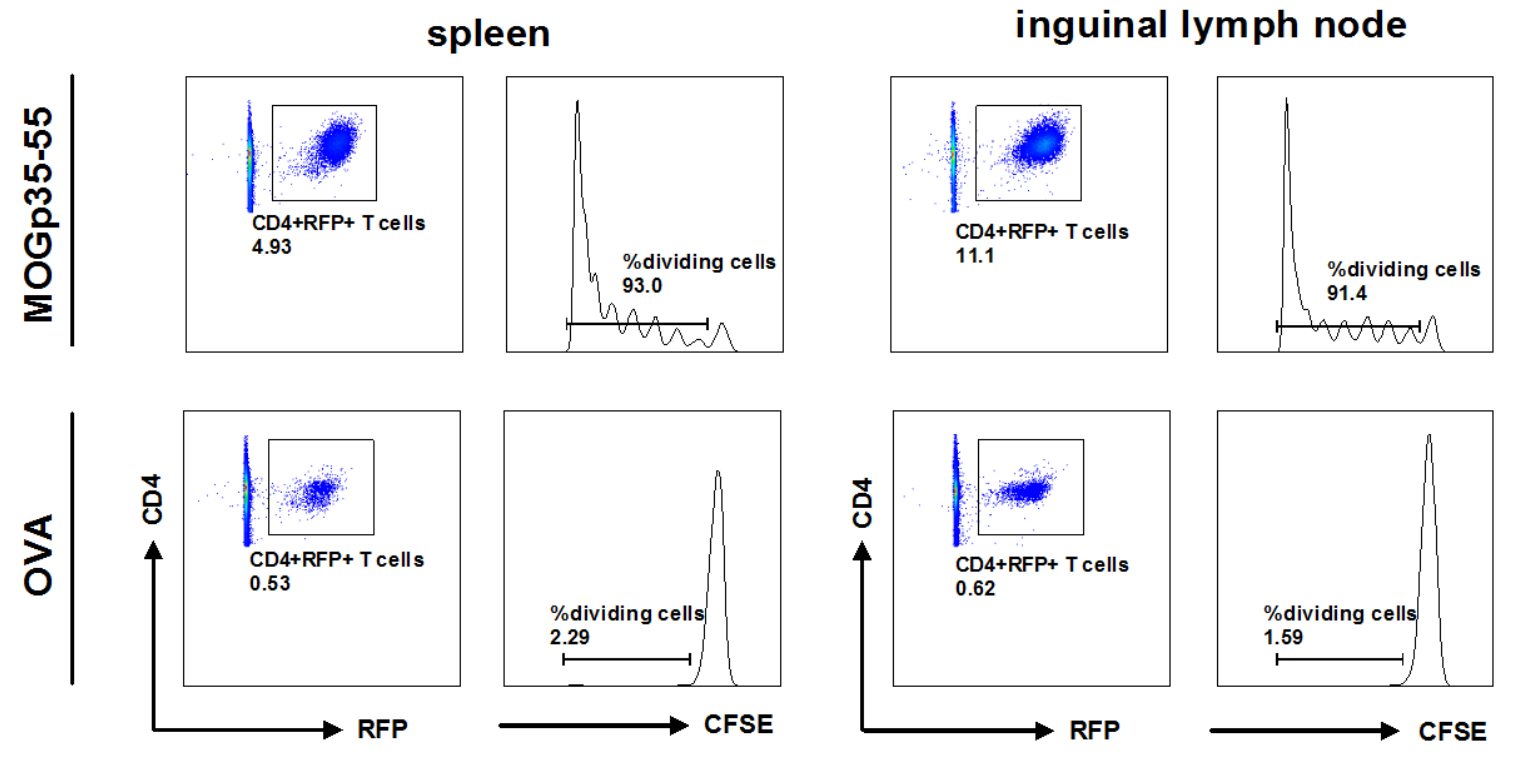

Figure 5: Transferred MOG-specific $T$ cells show antigen-specific proliferation. $\mathrm{RFP}^{+}$MOG-specific T cells were isolated from 2D2 mice, CFSE labeled and i.v. injected in C57bl/6 WT mice two days before immunization with either $\mathrm{MOG}_{\mathrm{p} 35-55}$ (upper row) or OVA protein (lower row). Five days p.i., single cell suspension from the spleen (left) and the draining lymph node (right) was prepared and labeled with an antibody against CD4 for flow cytometry analysis. Transferred MOGspecific T cells were identified by their simultaneous expression of CD4 and RFP (dotplots) and the proportion of CFSE dilution was examined with a histogram plot.

The transferred MOG-specific T cells exclusively proliferated after immunization with $\mathrm{MOG}_{\mathrm{p} 35-55}$ (Fig. 5 upper row) but not after immunization with a foreign antigen (Fig. 5 lower row) as measured by CFSE dilution. The specific amplification of MOG-specific $\mathrm{T}$ cells after immunization with $\mathrm{MOG}_{\mathrm{p} 35-55}$ can not only be demonstrated by the dilution of CFSE (histogram plots) but also by the frequency of $\mathrm{RFP}^{+} \mathrm{CD} 4^{+} \mathrm{T}$ cells among the total $\mathrm{CD}^{+} \mathrm{T}$ cell pool (dot plots). These basic observations ensure that we transferred naïve MOG-specific T cells which have to be activated in vivo like the endogenous $\mathrm{T}$ cell pool. In addition we ensure that the transferred MOG-specific $\mathrm{T}$ cells exclusively respond in an antigen-specific manner. 


\subsubsection{Characterization of the antigen-specific $T$ and $B$ cell interaction in vitro}

As we were interested in the antigen-specific interplay of $T$ and $B$ cells, we performed a series of in vitro experiments. We wondered whether the antigen presentation by $\mathrm{B}$ cells is sufficient to trigger $T$ cell proliferation in vitro. Therefore, we co-cultured $B$ cells with CFSE labeled $T$ cells in the presence of antigen and determined $T$ cell proliferation after 3 days. To investigate whether also B cells become activated upon T cell interaction, we determined MHC-II and CD86 expression on B cells. To study the contribution of antigen-specificity on the interplay of $T$ and $B$ cells, we co-cultured different antigen-specific $T$ and $B$ cells with increasing concentrations $(0,0.01,0.1,1$, $10 \mu \mathrm{g} / \mathrm{ml}$ ) of different antigens (MOG $\mathrm{p}_{35-55}$, rrMOG, NP-OVAL). We co-cultured either MOG-specific $T$ cells or OVA-specific $T$ cells (isolated from OVA-specific TCR transgenic mice (OT-II mice) [173]) with MOG-specific B cells or with NP-specific $B$ cells (isolated from B1.8 mice (in this study always termed "NP mice") [172]).

MOG-specific $T$ cells proliferated in the presence of MOG-specific $B$ cells and $M O_{p 35-55}$ but also in the presence of NP-specific B cells (Fig. 6 A left). In contrast, in the presence of rrMOG, only MOG-specific $B$ cells could trigger MOG-specific $T$ cell proliferation (Fig. $6 \mathrm{~A}$ middle). OVA-specific $\mathrm{T}$ cells proliferated neither in the presence of $M_{0} G_{p 35-55}$ nor in presence of rrMOG, but they responded to even very low NP-OVAL concentrations when presented by NP-specific B cells (Fig. 6 A right). The results regarding the $T$ cell proliferation led to the conclusion that a $T$ cell response can just be initiated in the presence of the appropriate antigen. Both, MOGspecific B cells and NP-specific B cells upregulated CD86 and MHC-II at high $M \mathrm{OG}_{\mathrm{p} 35-55}$ concentration, but exclusively in the presence of MOG-specific T cells (Fig. $6 \mathrm{~B}+\mathrm{C}$ left). In the presence of rrMOG, MOG-specific $\mathrm{B}$ cells upregulated CD86 and MHC-Il at very low antigen concentration, but just when they acquired $T$ cell help of the same antigen specificity (Fig. $6 \mathrm{~B}+\mathrm{C}$ middle). In contrast, NP-specific B cells upregulated CD86 and MHC-II in the presence of NP-OVAL and OVA-specific T cells, but also in the presence of antigen-unspecific $T$ cells (Fig. $6 \mathrm{~B}+\mathrm{C}$ right). In summary, the activation of $B$ cells was not as strictly as observed for the $T$ cells, as NP-specific B cells also upregulated MHC-II in the presence of an irrelevant antigen and of antigen-unspecific $T$ cells. However, the interaction of $T$ and $B$ cells resulted in an activation of both interaction partners. 
A


B
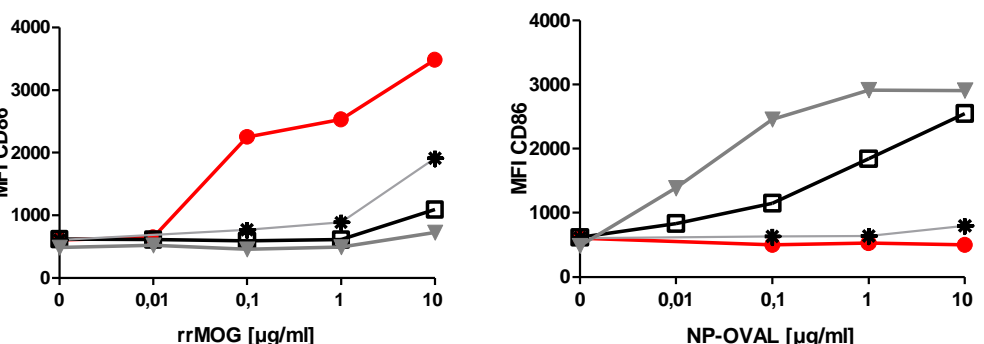

C
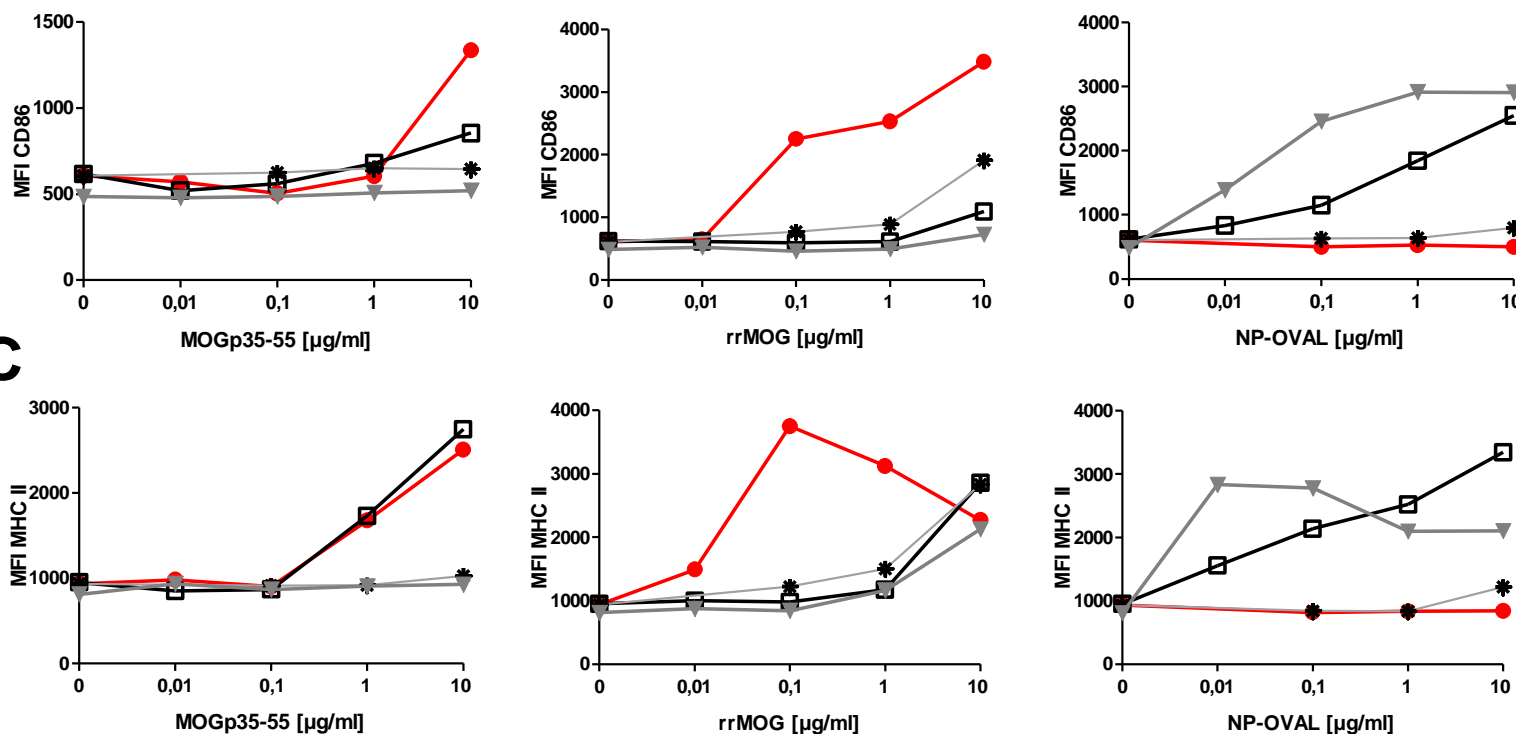

Figure 6: In vitro analysis of antigen-specific $\mathrm{T}$ and $\mathrm{B}$ cell interaction. $1 \times 10^{6}$ MOG-specific T cells were co-cultured with either $2 \times 10^{6}$ MOG-specific B cells (red line) or NPspecific B cells (open quad), as control OVA-specific T cells were co-cultured with MOG-specific B cells (black line) or NP-specific B cells (grey line) with increasing $\mathrm{MOG}_{\mathrm{p} 35-55}$ (left), rrMOG (middle) or NP-OVAL (right) concentrations $(0 ; 0.01 ; 0.1 ; 1 ; 10 \mu \mathrm{g} / \mathrm{ml})$ for $72 \mathrm{~h}$. (A) T cell proliferation was determined by CFSE dilution. $(B+C)$ Co-cultured B cells were stained for B220 and CD86 (B) or for B220 and MHC-II (C) and analyzed for their mean fluorescence intensity (MFI) of respective marker expression by flow cytometry. Shown is one representative experiment out of three independent experiments.

\subsubsection{MOG-specific $B$ cells accelerate the disease onset in actively induced}

\section{EAE}

To investigate whether the cell transfer had a direct effect on the course of EAE, we transferred $8 \times 10^{6}$ MOG-specific T cells, 5-10 $\times 10^{6}$ MOG-specific B cells or both together into C57bl/6 WT mice followed by immunization with $\mathrm{MOG}_{\mathrm{p} 35-55}$ and monitored the clinical outcome for 18 days. 



Figure 7: Clinical outcome of MOG-specific $\mathrm{T}$ and/or MOG-specific B cell transfer into WT mice and immunization with MOG $_{p 35-55}$. MOG-specific $T$ and $B$ cells were isolated from respective donor mice and i.v. transferred into WT mice followed by immunization with $\mathrm{MOG}_{\mathrm{p} 35-55}$ two days later. (A) EAE disease course of $T$ and $B$ cell (grey line), $T$ cell (black line) or PBSsupplemented (red line) mice. (B) Day of onset of each animal per T or TB group. Shown are two pooled experiments with each 8 (T group), 10 (TB group) and 6 (PBS) animals. (C) EAE disease course of B cell (black broken line) and PBS (red line) supplemented mice. Shown is one experiment with 7 mice per group. Clinical graphs show mean \pm SEM. Non-significant differences between means are indicated: ns $\mathrm{p}>0,05$, Mann-Whitney nonparametric $t$ test.

Control PBS-injected WT mice started to develop first clinical signs of EAE at about day 10-11 post immunization and peak disease severity on day 15 followed by a partial recovery (Fig. $7 \mathrm{~A}+\mathrm{C}$, red line). The transfer of either MOG-specific $\mathrm{T}$ cells (Fig. 7 A, black line), MOG-specific B cells (Fig. $7 \mathrm{C}$, black broken line) or both together (Fig. 7 A, grey line) accelerated the disease onset and also exacerbated disease severity as almost all animals had to be sacrified during the experiment. Though the transfer of MOG-specific $T$ cells and MOG-specific B cells alone each had an effect on the day of disease onset, the simultaneous transfer of both cell types could just slightly, but not significantly, promote the clinical outcome as depicted in direct comparison of the day of onset (Fig. 7 B). We assumed that the contribution of endogenous $\mathrm{T}$ and $\mathrm{B}$ cells to disease severity make it difficult to further increase the clinical outcome. To overcome this problem, we copied the 
model to OT-II mice as hosts, as they harbor $>90 \%$ of OVA-specific T cells enabling us to minimize endogenous $T$ cell effects as we know from in vitro experiments, that OVA-specific T cells do not respond to MOG antigens (Fig. 6 A left+ middle). To minimize endogenous $T$ and $B$ cell effects, we crossed OT-II mice to NP mice (termed OT-II $\times$ NP), resulting in host mice mainly harboring OVA-specific T cells and NP-specific B cells.
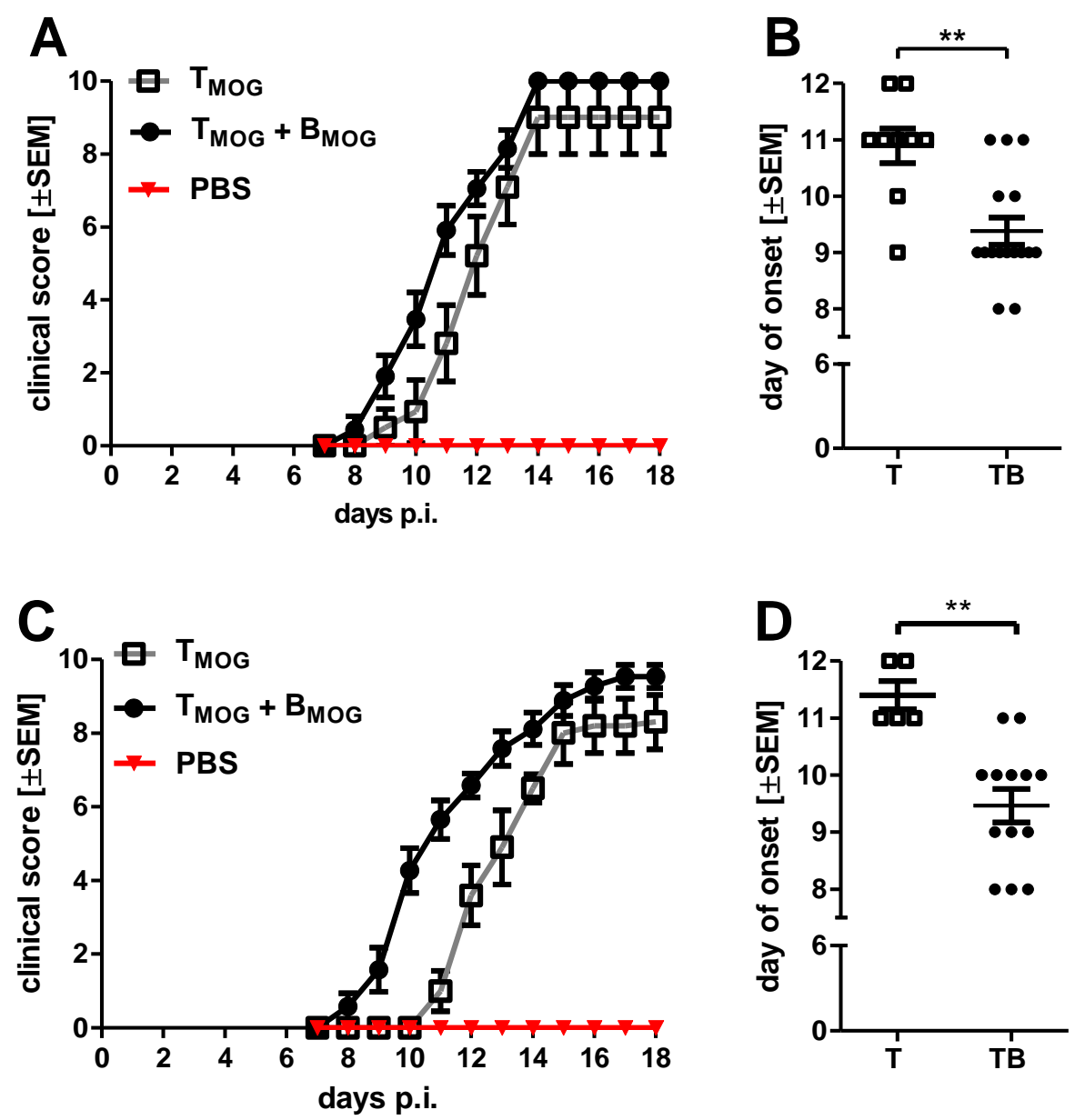

Figure 8: MOG-specific B cells accelerate the onset of EAE in OT-II and OT-II x NP mice. MOG-specific $T$ and $B$ cells were isolated from respective donor mice and i.v. transferred into OT-II $(\mathbf{A}, \mathbf{B})$ or OT-II x NP (C,D) mice followed by immunization with $\mathrm{MOG}_{\mathrm{p} 35.55}$ two days later. (A) EAE disease course of T (grey line), TB (black line) or PBS supplemented (red line) OT-II mice. (B) Day of onset of each animal per T or TB group. Shown are four pooled experiments with 14 (T group), 16 (TB group) and 6 (PBS) animals. (C) EAE disease course of T (grey line), TB (black line) or PBS supplemented (red line) OT-II x NP mice. (D) Day of onset of each animal per T or TB group. Shown are three pooled experiments with each 14 (T group), 15 (TB group) and 3 (PBS) animals. Clinical graphs show mean \pm SEM, healthy mice were not included in the analysis. Significant differences between means are indicated: ${ }^{* *} p<0,01$, Mann-Whitney nonparametric $t$ test.

Both, OT-II and OT-II $\times$ NP mice were not susceptible for EAE induction without previous cell transfer (Fig. $8 \mathrm{~A}+\mathrm{C}$ red line). However, the resistance could be overcome by the transfer of MOG-specific $T$ cells (Fig. $8 A+C$ grey line) or the 
simultaneous transfer of MOG-specific $T$ and $B$ cells (Fig. $8 A+C$ black line). In addition, the disease onset could be significantly accelerated by the simultaneous transfer of MOG-specific T and B cells in OT-II (Fig. 8 B, Table 4) and in OT-II x NP mice (Fig. $8 \mathrm{D}$, Table 4), leading to the conclusion that MOG-specific $B$ cells had a disease promoting effect in this experimental setting. Furthermore, the additional transfer of MOG-specific B cells increased the incidence for EAE (in OT-II: T: $61.5 \%$, TB: $100 \%$; in OT-II x NP: T: $35.7 \%$, TB: $86.7 \%$ ) as summarized in Table 4. Nevertheless, the disease severity was not increased by the additional transfer of MOG-specific B cells, because as soon as T cell-injected mice developed first clinical signs, the clinical progress was similar to that observed in mice receiving $T$ and $B$ cells. Furthermore, cell transfer followed by active immunization always resulted in sacrifice of the animals due to a clinical score $>7$, thus making it impossible to study the recovery phase. Preliminary data also reveal the same effect of MOG-specific $B$ cells in rrMOG-induced EAE (data not shown).

Table 4: Influence of cell transfer into OT-II and OT-II $x$ NP mice on EAE susceptibility

\begin{tabular}{cccc} 
Host & Cell transfer & $\begin{array}{c}\text { Mean day of onset } \\
( \pm \text { SEM) }\end{array}$ & Incidence \\
\hline \multirow{2}{*}{ OT-II } & T cells & $10.9 \pm 0.3$ & $61,5 \%$ \\
& T+B cells & $9.4 \pm 0.24$ & $100 \%$ \\
\multirow{2}{*}{ OT-II x NP } & T cells & $11.4 \pm 0.24$ & $35,7 \%$ \\
& T+B cells & $9.5 \pm 0.3$ & $86,7 \%$ \\
\hline
\end{tabular}

Although several publications suggest a dispensable role for $B$ cells in MOG peptideinduced EAE, our established OT-II EAE model as well as the OT-II x NP EAE model reveal an effect of MOG-specific $B$ cells after $M_{0 O G_{p 35-55}}$ immunization. As we observed a disease promoting effect reflected by an earlier disease onset and a higher incidence in both models, we rather suggest a pro-inflammatory role for the transferred $B$ cells than a role for $B$ cells as regulatory cells. We now wanted to clarify the underlying mechanisms utilized by $B$ cells to promote the development of EAE.

Most of the experiments described in the PhD-thesis were performed in OT-II mice and critical findings were repeated in OT-II $\times$ NP mice as the B cell-mediated effect was more pronounced in this model. For our further analysis, we always compared 
the phenotype of T cells injected alone (in graphs always depicted as "T") with the T cells which were injected together with MOG-specific B cells (in graphs always depicted as "TB").

\subsection{Influence of MOG-specific B cells during the activation phase of}

\section{EAE}

In contrast to other professional antigen-presenting cells like dendritic cells or macrophages, B cells express an antigen-specific B cell receptor on their surface enabling them to capture even low amounts of antigen, process it and present it bound on MHC-II molecules to $\mathrm{CD}^{+} \mathrm{T}$ cells. Therefore, several publications suggest a role for $B$ cells as antigen-presenting cells during $E A E[78,79,139]$. By presenting antigen to $T$ cells, $B$ cells can induce $T$ cell proliferation, activation or their differentiation into a helper $\mathrm{T}$ cell direction, which is crucial for the development of EAE. In active EAE, myelin-specific $T$ cells get activated within the first 4-5 days post disease induction in secondary lymphoid organs. According to the side of immunization, different lymph nodes arise as draining lymph nodes where $\mathrm{T}$ cellpriming predominantly occurs. The subcutaneous immunization into the tail base determines the inguinal (ing LN) and the paraaortic lymph nodes (para LN) as draining lymph nodes, whereas the axillary (ax LN) and the cervical lymph nodes (cer LN) are non-draining lymph nodes. To clarify whether MOG-specific B cells exert their disease-promoting function within the first four days after immunization, we characterized the injected MOG-specific T cells in terms of proliferation, activation and differentiation within the first four days post immunization (schematically depicted in Fig. 9).

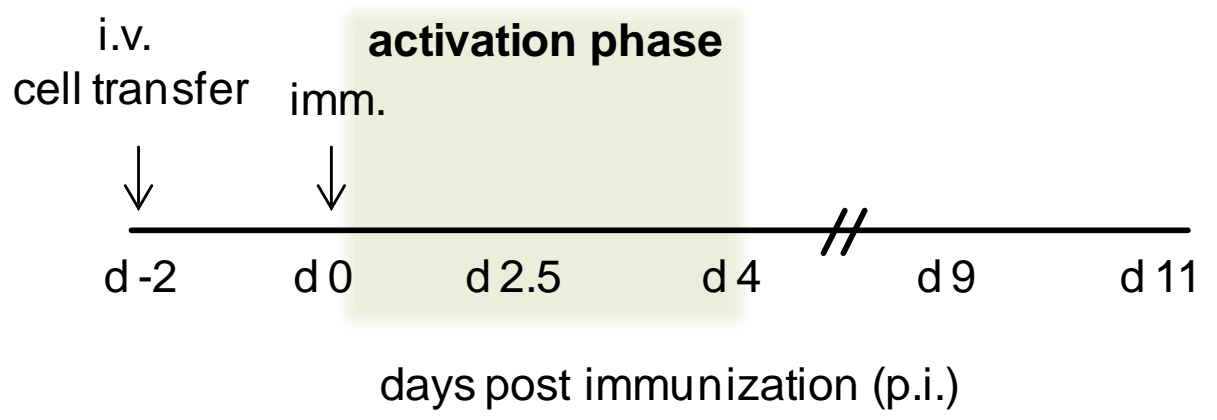

Figure 9: Schematic overview of experimental design with focus on the activation phase. MOG-specific T cells +/- MOG-specific B cells were i.v. transferred into OT-Il recipients (d-2) and immunized with $\mathrm{MOG}_{\mathrm{p} 35-55} 2$ days later $(\mathrm{d} 0)$. Cells were analyzed either $2.5 \mathrm{~d}$ or $4 \mathrm{~d}$ p.i. 


\subsubsection{MOG-specific B cells do not enhance antigen-specific $T$ cell proliferation in vivo}

Although some publications suggest a dispensable role for $\mathrm{B}$ cells in enhancing $\mathrm{T}$ cell proliferation during EAE [139, 154], we wanted to investigate whether MOG-specific $B$ cells enhance the proliferation of MOG-specific T cells in our experimental model. Freshly isolated RFP-expressing MOG-specific T cells were labeled with CFSE and injected either alone or together with MOG-specific B cells in OT-II recipients followed by immunization with $\mathrm{MOG}_{\mathrm{p} 35-55}$ two days later. The CFSE dilution of the injected MOG-specific $T$ cells was analyzed ex vivo $2 \mathrm{~d}, 2.5 \mathrm{~d}$ and $4 \mathrm{~d}$ post immunization by flow cytometry. Both, T cells injected alone or together with MOG-specific B cells started to proliferate at about $48 \mathrm{~h}$ to $60 \mathrm{~h}$ post immunization in the draining lymph nodes (Fig $10 \quad \mathrm{~A}+\mathrm{B}$ ) and displayed similar proliferative responses during the subsequent time points in the draining lymph nodes, non-draining lymph nodes and in the spleen (Fig. $10 \mathrm{~B}$ ). As it is possible that strongly proliferated cells were intangible with this analysis because they have already left the lymph node, we additionally determined the number of transferred $\mathrm{T}$ cells in the different lymph nodes, in the spleen and in the blood, as well as the frequency of RFP ${ }^{+} \mathrm{CD}^{+} \mathrm{T}$ cells among the total $\mathrm{CD}^{+} \mathrm{T}$ cell pool four days post immunization (Fig. $10 \mathrm{C}+\mathrm{D}$ ). At this time point we could not detect any differences in the cell number, neither in the secondary lymphoid organs, nor in the blood (Fig. $10 \mathrm{C}$ ), which was consistent with the frequency of RFP ${ }^{+}$T cells among total $\mathrm{CD}^{+} \mathrm{T}$ cells (Fig. $10 \mathrm{D}$ ). 
A
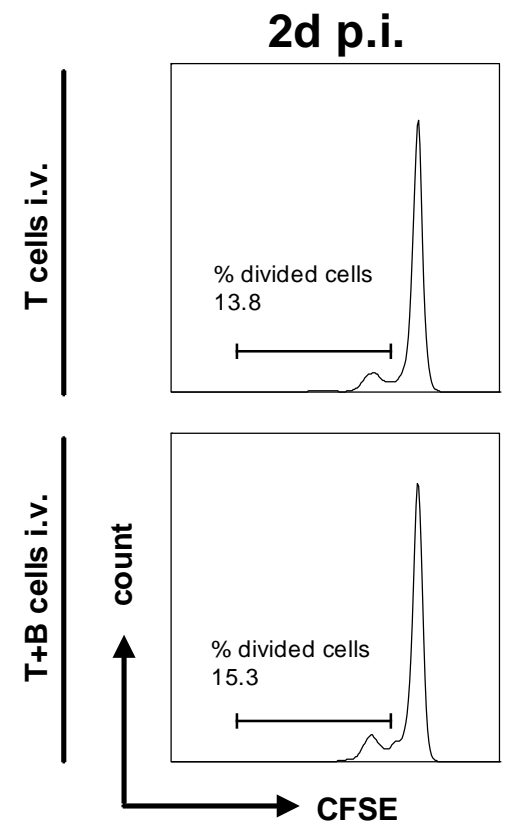

2.5d p.i.
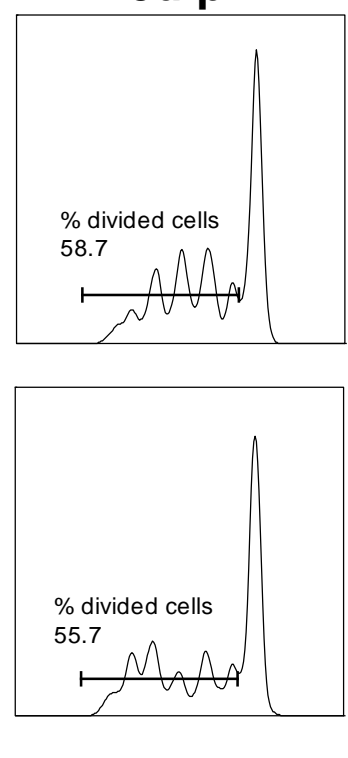

4d p.i.
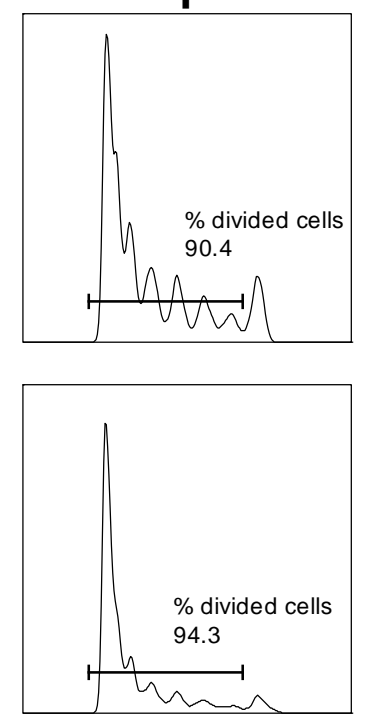

B
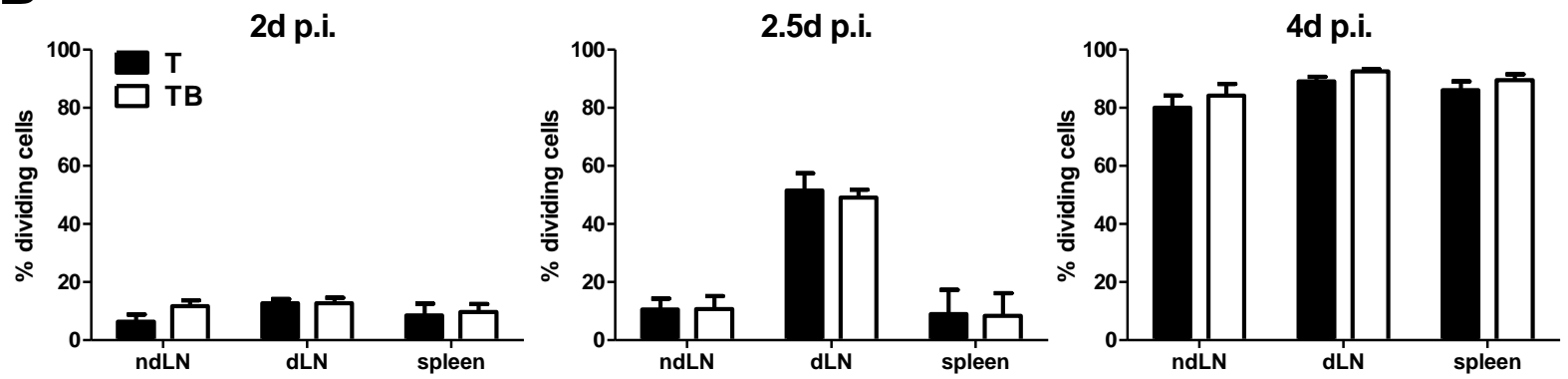

C
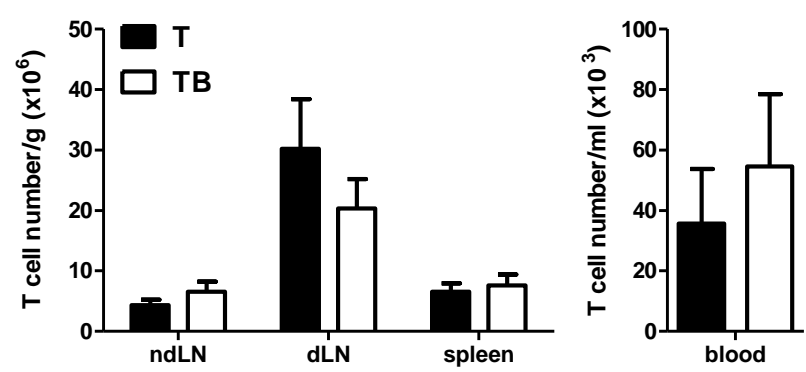

D

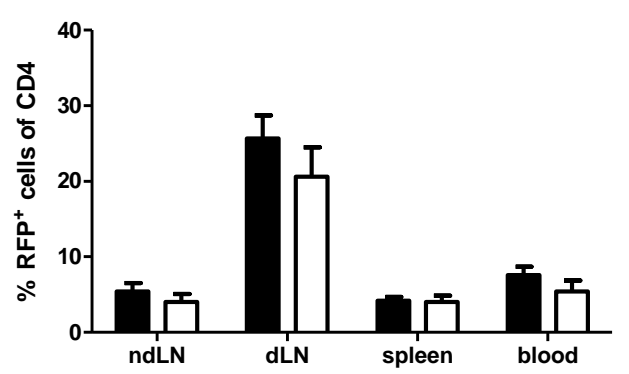

Figure 10: MOG-specific $B$ cells do not enhance $T$ cell proliferation in vivo. (A+B) CFSE-labeled MOG-specific T cells were ex vivo analyzed for CFSE dilution via flow cytometry (A) representative flow cytometry histogram plots of T cell CFSE dilution analyzed from the draining lymph node 2, 2.5 and 4 days p.i. either injected alone (upper row) or together with MOG-specific B cells (lower row). (B) Quantification of CFSE dilution of T cells analyzed from different lymph nodes and spleen of three pooled independent experiments, determination of \% divided cells was performed like depicted in A. (C) absolute numbers of transferred MOG-specific T cells were determined like described in chapter 2.2.8 for lymph nodes, spleen and blood 4d p.i. (D) Frequency of RFP ${ }^{+} \mathrm{CD}^{+}$ among total $\mathrm{CD} 4^{+}$was determined with flow cytometry $4 \mathrm{~d}$ p.i.. Data represent $>4$ animals per group. Data are depicted as mean \pm SEM. 


\subsubsection{MOG-specific B cells do not enhance antigen-specific $T$ cell activation in}

\section{vivo}

Activated $T$ cells can be discriminated form naïve $T$ cells by their expression of different surface molecules. Upon antigen encounter within the lymph node, $T$ cells downregulate their CD62L expression enabling them to leave the lymph node and migrate into the blood. CD69 is one of the earliest surface markers which is upregulated by $\mathrm{T}$ cells following activation. CD44 is an adhesion molecule and one characteristic marker of antigen-experienced T cells. In order to test whether the in vivo interaction of MOG-specific $T$ and $B$ cells increase the frequency of activated $T$ cells, we stained them with fluorescently labeled antibodies against CD69, CD62L and CD44 and analyzed the percentage of positive cells with flow cytometry four days p.i..
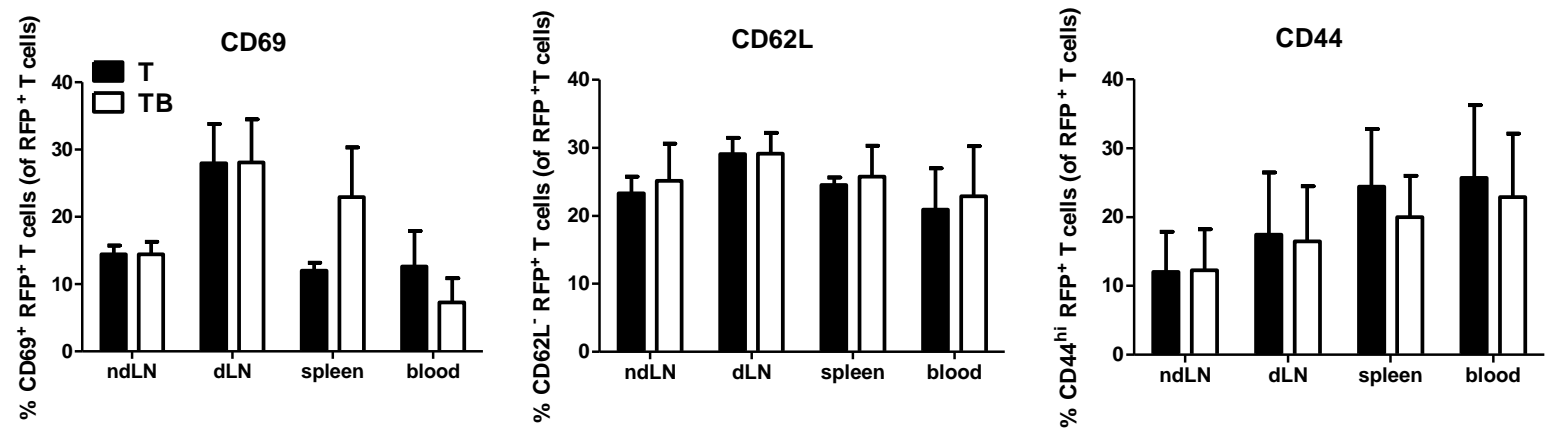

Figure 11: MOG-specific $B$ cells do not influence $T$ cell activation in vivo. Single cell suspensions of different lymph nodes, spleen and blood of mice were stained against CD69, CD62L and CD44 4d p.i.. Injected MOG-specific T cells were identified as CD4 ${ }^{+}$RFP $^{+}$. Figure depicts the percentage of positive (in case of CD62L: negative) cells among the $\mathrm{CD}^{+} \mathrm{RFP}^{+}$cell pool. Data represent two independent experiments with 4 animals per group. Data are depicted as mean \pm SEM.

The presence of MOG-specific B cells did not increase the frequency of $\mathrm{CD}^{2} \mathrm{~L}^{-}$ $\mathrm{RFP}^{+} \mathrm{T}$ cells (Fig. 11 middle) or of $\mathrm{CD} 44^{\text {hi }} \mathrm{RFP}^{+} \mathrm{T}$ cells (Fig. 11 right) within the different lymph nodes, the spleen or the blood. In contrast, there was a slight increase of $\mathrm{CD}_{69}{ }^{+} \mathrm{T}$ cells when injected together with MOG-specific $\mathrm{B}$ cells within the spleen (Fig. 11 left), but not in the lymph nodes or in the blood and it is questionable whether this difference accounts for the accelerated disease onset. Nevertheless, in comparison to activation marker expression of $T$ cells before transfer (Fig. 4), the cells became activated upon EAE induction within the first four days p.i.. 


\subsubsection{MOG-specific B cells do not promote early antigen-specific $T$ cell differentiation in vivo}

Upon antigen encounter within a pro-inflammatory milieu, naïve $\mathrm{CD}^{+} \mathrm{T}$ cells differentiate into helper $\mathrm{T}$ cells mainly characterized by their produced effector cytokines. TH1 and TH17 T cells are the critical helper T cell subsets in EAE. B cells have been shown to amplify $T$ helper cell subsets in EAE and thus, contribute to disease pathogenesis $[56,78,79,139]$. We therefore wanted to investigate whether the additional transfer of MOG-specific B cells influence the cytokine production of the transferred $\mathrm{T}$ cells in our experimental model. For this purpose, we sorted the transferred MOG-specific T cells according to their RFP and CD4 expression from the draining lymph nodes 2.5 days p.i. or from draining lymph nodes and spleen 4 days p.i. and determined the mRNA expression level of IFN $\gamma$, IL-17A and GM-CSF via RT-PCR. Sorted $\mathrm{RFP}^{+} \mathrm{CD} 4^{+} \mathrm{MOG}$-specific $\mathrm{T}$ cells from the draining lymph nodes of $\mathrm{T}$ and $\mathrm{B}$ cell-injected mice did not show an increased IFN $\gamma$ and IL-17A expression 2.5 days p.i. (Fig. $12 \mathrm{~A}$ ). On that time point, GM-CSF mRNA was not detectable in both groups. In addition, mRNA expression of IFN $\gamma$, IL-17A and GM-CSF was not statistically different 4 days p.i. (Fig. 12 B). Nevertheless, in comparison to mRNA expression of $\mathrm{T}$ cells before transfer (naïve $\mathrm{T}$ cells, grey bars), in vivo activated MOG-specific T cells showed an upregulation of IFN $\gamma$, IL-17A and GM-CSF within the first four days after immunization (Fig. 12 B). 
A
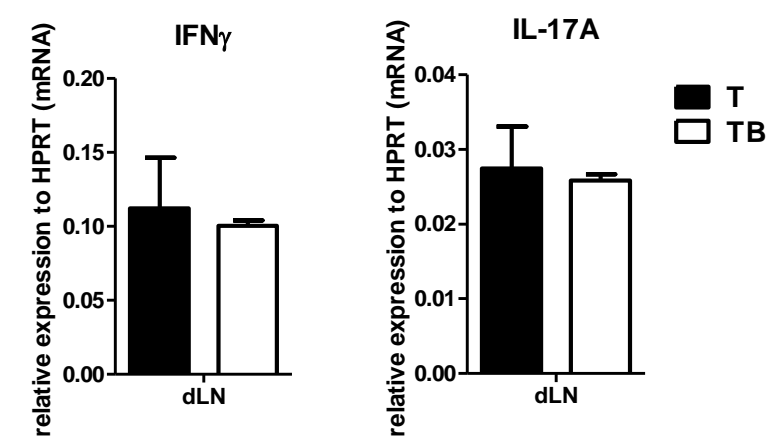

B
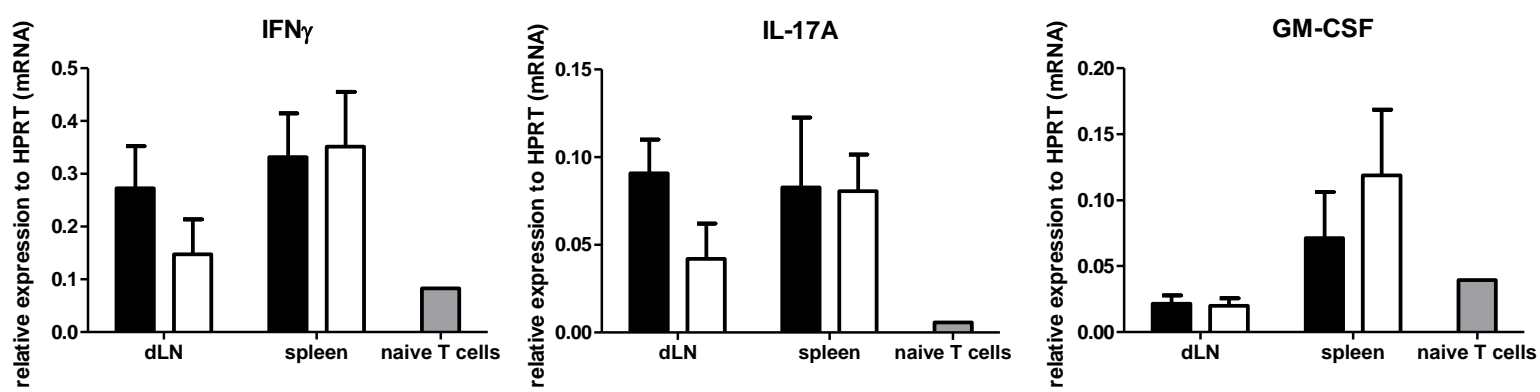

Figure 12: MOG-specific $B$ cells do not increase the expression of $\mathrm{TH} 1$ and $\mathrm{TH} 17$ specific cytokines on mRNA level. Cytokine expression (mRNA) of sorted RFP+CD4 ${ }^{+} \mathrm{T}$ cells $2.5 \mathrm{~d}$ p.i. (A) or $4 \mathrm{~d}$ p.i. (B) or from RFP ${ }^{+} \mathrm{CD}^{+} \mathrm{T}$ cells before transfer (grey bar). Graphs show the expression of respective mRNA relative to the endogenous housekeeping gene hypoxanthine phosphoribosyltransferase 1 (HPRT). Data in (A) and (B) represent 3 and 4 animals per group, respectively. Data are depicted as mean \pm SEM.

To further evaluate whether MOG-specific B cells affected $T$ cell differentiation on the protein level, we restimulated total lymph node cells and splenocytes isolated from $T$ cell or $T$ and $B$ cell-injected OT-II mice $4 d$ p.i. in vitro with increasing $M_{0 G} G_{p 35-55}$ antigen concentrations. We assessed the amounts of secreted IFN $\gamma$, IL-17A and GMCSF after 72h in the supernatant via ELISA. As the frequency of RFP ${ }^{+}$MOG-specific $\mathrm{T}$ cells was similar between the two groups at that time point (Fig. $10 \mathrm{D}$ ), we assumed that an observed effect was due to an increased cytokine production or an increased number of differentiated $T$ cells, but not due to a higher number of MOGspecific $T$ cells in total. Total lymph node cells and splenocytes from $T$ and $B$ cellinjected mice did not show an enhanced reactivity in response to $M_{0 G_{p 35-55}}$ in comparison to restimulated cells from $T$ cell-injected mice. They neither showed an enhanced sensitivity to lower antigen concentrations, nor a higher production of IFN $\gamma$ (Fig. $13 \mathrm{~A}$ ), IL-17A (Fig. $13 \mathrm{~B}$ ) or GM-CSF (Fig. $13 \mathrm{C}$ ) at the same antigen concentration. 
Therefore, we exclude a critical role for $B$ cells in enhancing $T$ cell proliferation, activation and differentiation within the activation phase of EAE.

A

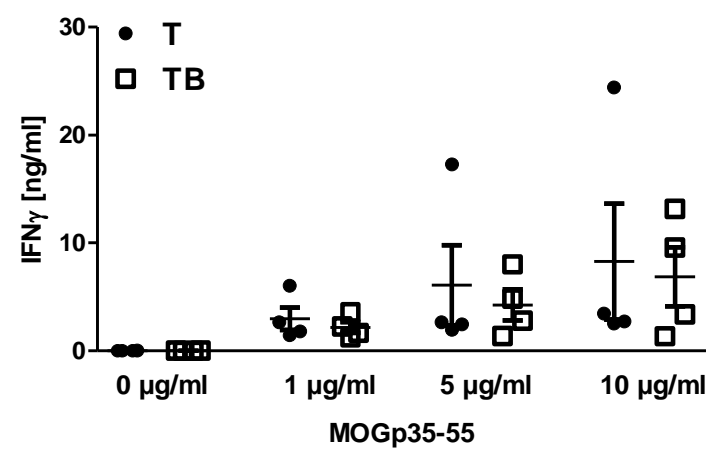

B

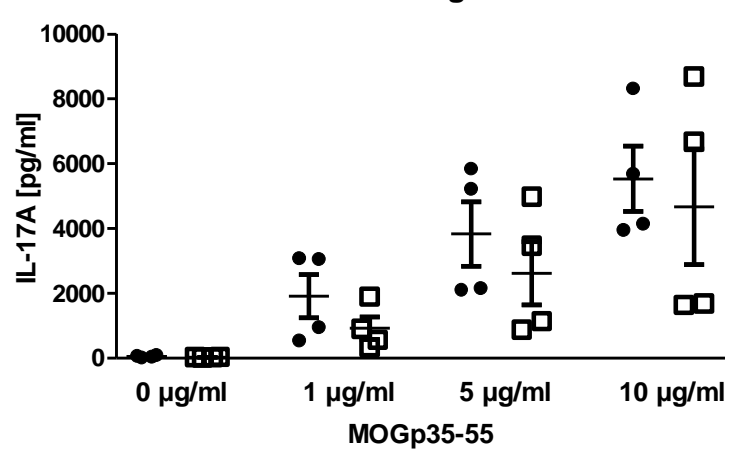

C

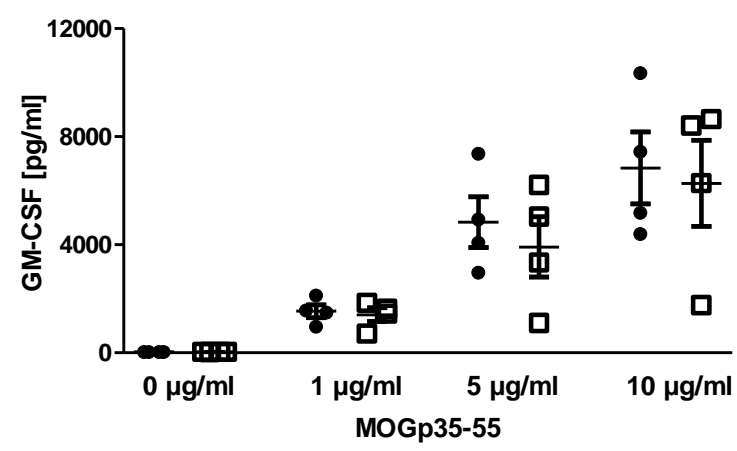

IFN $\gamma$ spleen



IL-17A spleen

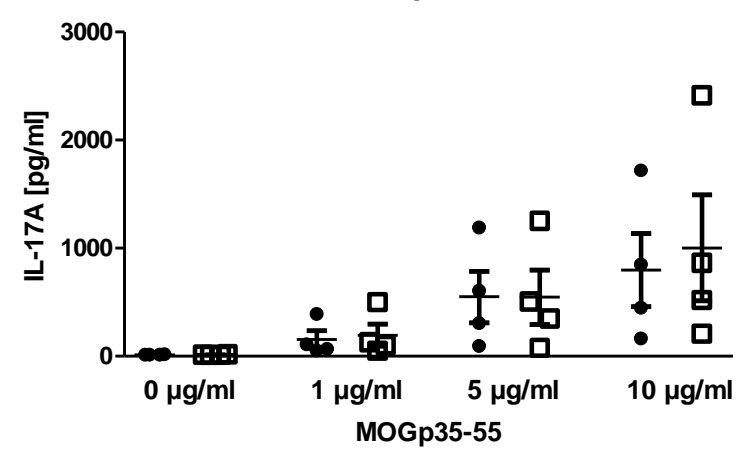

GM-CSF spleen



Figure 13: MOG-specific $B$ cells do not enhance reactivity of $T$ cells to $M O G_{p 35-55}$. Total draining lymph node cells and splenocytes were isolated $4 d$ p.i. from $T$ cell (black circles) or $T$ and $B$ cell (white quad) injected mice and restimulated in vitro with increasing $M O G_{p 35-55}$ concentrations for $72 \mathrm{~h}$. Supernatant was collected and the amount of IFN $\gamma$ (A), IL-17A (B) and GMCSF (C) was determined by ELISA. Each symbol represents an individual mouse out of two independent experiments, with the bar indicating the mean \pm SEM. 


\subsection{Characterization of $\mathrm{T}$ cell behavior at the onset of $\mathrm{EAE}$}

As we could not detect any obvious differences in the $\mathrm{T}$ cell activation, in their proliferation and in their $T$ helper cell differentiation during the activation phase of actively induced EAE, we assumed that the MOG-specific B cells exert their proinflammatory functions at later time points. Hence, we characterized the transferred $T$ cells at day 9 p.i., the disease onset of the $T$ and B cell-injected OT-II mice (as schematically depicted in Fig. 14). We were interested whether $B$ cells might affect $T$ cell activation or the peripheral $\mathrm{T}$ cell numbers shortly before the appearance of first clinical signs. Furthermore, as it is suggested that MOG-specific B cells enhance $T$ cell differentiation, we also investigated whether the presence of MOG-specific $B$ cells influence MOG-specific T cell differentiation at later time points. Moreover, we were intending to uncover the reason why $T$ and $B$ cell-injected mice develop EAE earlier than T cell-supplemented mice. Therefore, we focused on early events in the CNS (before appearance of clinical signs) to figure out whether B cells play a critical role in $\mathrm{T}$ cell reactivation within the CNS or whether they promote $\mathrm{T}$ cell infiltration by influencing their migration behavior.

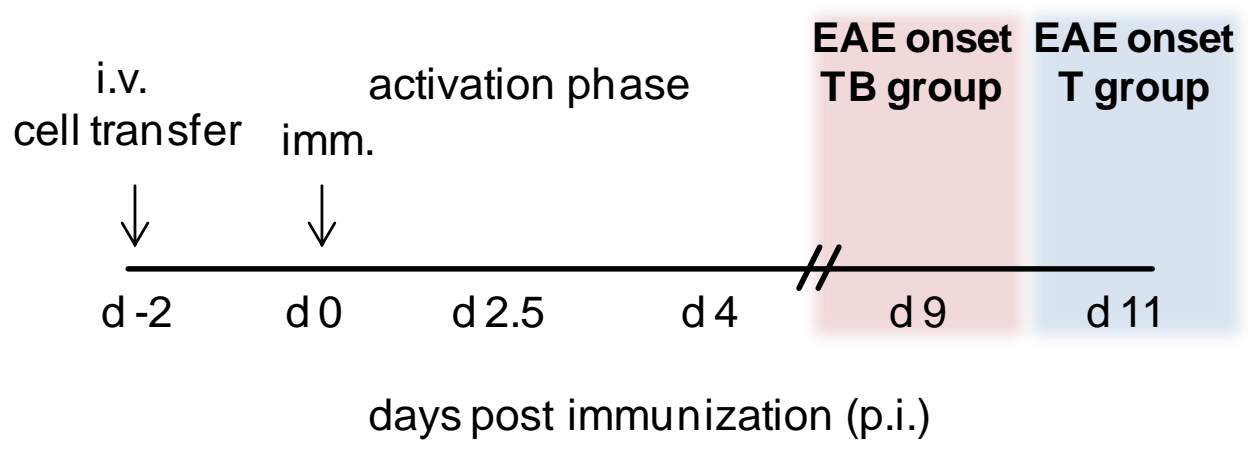

Figure 14: Schematic overview of experimental design with focus on the onset of EAE. MOG-specific T cells +/- MOG-specific B cells were i.v. transferred into OT-II recipients (d-2) and immunized with $\mathrm{MOG}_{\mathrm{p} 35-55} 2$ days later (d0). Cells were analyzed $9 \mathrm{~d}$ p.i. (onset of TB group) or d11 (onset of T group). 


\subsubsection{MOG-specific $B$ cells do not influence antigen-specific $T$ cell number and activation before onset}

As we could not detect any difference in the T cell phenotype within the activation phase (2-4d p.i.), we wanted to investigate whether this observation changed during the progress of the pre-clinical phase, precisely on the day of EAE onset. We were interested whether the $T$ cell phenotype of healthy animals with extravasated $T$ cells (shortly before appearance of clinical symptoms) differs from these of animals without extravasated $T$ cells. In order to determine the presence of extravasated T cells, my colleague Tanja Litke performed two-photon intravital imaging of the spinal cord. With this technique it is possible to visualize the fluorescently labeled $T$ cells, both extravasated or intraluminal within the leptomeningeal vessels of the spinal cord. Starting from day 8 p.i., we analyzed 12 animals in total $(6 \times T, 6 \times T B)$ for the following 2 days. During this time, 4 animals of the TB group had extravasated T cells without clinical manifestation of disease and one animal with a clinical score. In contrast, no animal of the T group had a clinical score and just one had extravasated T cells (Fig. $15 \mathrm{~A}$ ). For further flow cytometry analysis, the $\mathrm{RFP}^{+}$cell number within the CNS and peripheral lymphoid organs of $T$ cell-injected animals without extravasated $T$ cells and $T$ and $B$ cell-injected animals with extravasated $T$ cells was compared. We could detect a difference in cell number within the CNS (Fig. $15 \mathrm{~B}$ ), but this was neither reflected in the blood, nor in different non-draining (ax: axillary LN, cer: cervical LN), draining lymph nodes (ing: inguinal LN, para: paraaortic LN) nor in the spleen (Fig. 15 B). In line with this, also the frequency of $\mathrm{RFP}^{+} \mathrm{T}$ cells among the $\mathrm{CD} 4^{+} \mathrm{T}$ cell pool was not different between the two groups (Fig. $15 \mathrm{C}$ ). We could further not detect a striking difference in the frequency of $\mathrm{CD} 69^{+} \mathrm{RFP}^{+} \mathrm{T}$ cells, $\mathrm{CD}^{-} \mathrm{L}^{-} \mathrm{RFP}^{+} \mathrm{T}$ cells or CD44 ${ }^{\text {hi }} \mathrm{RFP}^{+} \mathrm{T}$ cells between the two groups, neither in the blood, nor in the different lymph nodes or in the spleen (Fig. 15 D). From these observations, we conclude that MOG-specific B cells did not amplify the peripheral MOG-specific $T$ cells or increase the frequency of activated $T$ cells. 
A

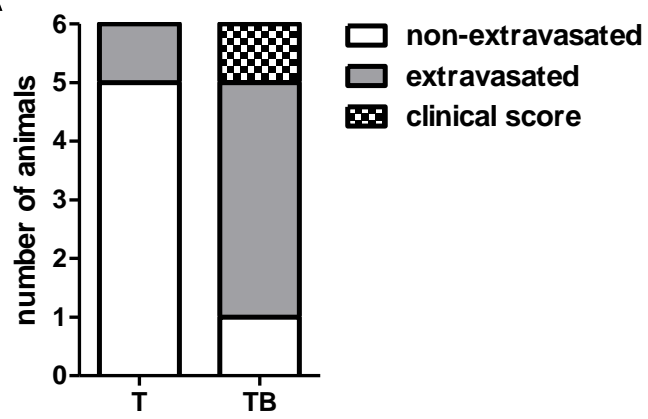

B

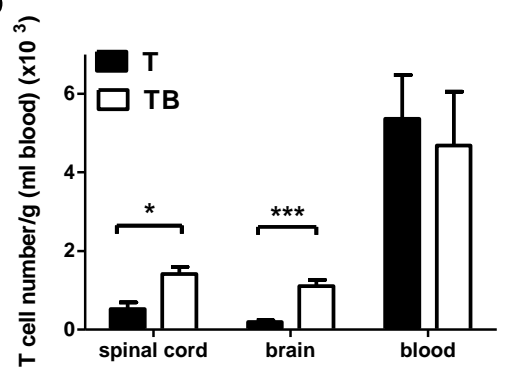

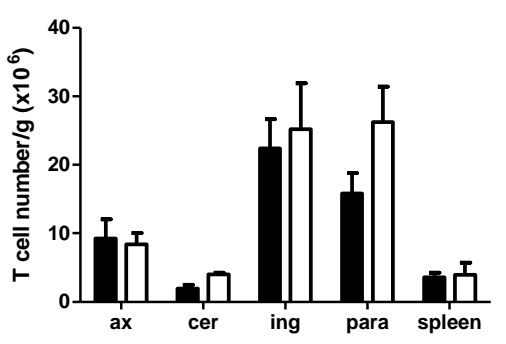


Figure 15: MOG-specific $B$ cells do not influence $T$ cell number or activation at the onset of the disease. Injected OT-II mice were evaluated for the presence of infiltrated RFP' $\mathrm{T}$ cells at the onset of EAE by two-photon microscopy and further analyzed with flow cytometry 8-10d p.i.. (A) Number of animals with non-extravasated (white), extravasated (grey) T cells or clinical score (dotted). (B-D) For analysis, $T$ cell-injected animals without extravasated cells ( $T$, black bars) were compared to $T$ and B cell-injected animals with extravasated cells (TB, white bars) (B) Number of transferred MOG-specific T cells was determined like described in chapter 2.2.8. for spinal cord, brain, blood, lymph nodes and spleen (C) Frequency of $\mathrm{RFP}^{+} \mathrm{CD4}^{+}$among total $\mathrm{CD}^{+}$was determined with flow cytometry. (D) Flow cytometry analysis of CD69, CD62L and CD44 expression of $C D 4^{+} \mathrm{RFP}^{+} \mathrm{T}$ cells. Data represent $>4$ animals per group and are depicted as mean \pm SEM, Significant differences are depicted: ${ }^{*} \mathrm{p}<0.05,{ }^{* \star *} \mathrm{p}<0.001$, unpaired students $t$ test 


\subsubsection{MOG-specific B cells do not promote $T$ cell differentiation before disease onset}

We know from our investigations concerning the cytokine expression of $T$ cells within the activation phase that $B$ cells are dispensable for the initial differentiation of naïve T cells into either TH1 or TH17 cells. Nevertheless, it is possible that they are crucial during the later time points as also suggested by other studies [56, 139]. In order to assess whether MOG-specific B cells increase pro-inflammatory cytokine production of the transferred MOG-specific $T$ cells at later time points, we sorted them shortly before the onset of EAE according to their simultaneous RFP and CD4 expression from peripheral lymphoid organs and from the blood and performed RT-PCR. To asses cytokine secretion on protein level, we restimulated total draining lymph node cells and splenocytes of either T cell-injected or T and B cell-injected OT-II mice 9 days p.i. in vitro with increasing $M O_{\mathrm{p} 35-55}$ concentrations for $72 \mathrm{~h}$ and quantified IFN $\gamma$, IL-17A and GM-CSF from the supernatant by ELISA. We could not detect any difference in the expression of IFN $\gamma$, IL-17A and GM-CSF on mRNA level of T cells, which were injected alone (Fig. 16 A black bars) or together with MOG-specific $B$ cells (Fig. $16 \mathrm{~A}$ white bars). As a lot of publications compare the cytokine expression between sick and healthy animals, we also included a third group, $T$ cells sorted from $T$ and $B$ cell-injected mice with clinical score (Fig. 16 red bars), in our analysis. Interestingly, even MOG-specific $T$ cells sorted from sick animals did not show an increased cytokine mRNA expression level. This was, at least in part, in contrast to the data we obtained by quantification of the different cytokines after restimulation of lymph node cells or splenocytes in vitro. Here, we could detect an increased amount of IFN $\gamma$ of restimulated lymph node cells from sick T and B cell-injected mice (Fig. 16 $B$ left, red circles) and an even more pronounced IL-17A amount of restimulated splenocytes (Fig. $16 \mathrm{C}$ right). These contradictory results lead to the conclusion, that the expression of cytokines is not upregulated on the single cell level during EAE, but rather that the number of cytokine-expressing cells increased in total. Nevertheless, this could not account for the accelerated disease onset, because there was no difference when we compared cells isolated from healthy animals, neither in the amount of IFN (Fig. 16 B), IL-17A (Fig.16 C) nor of GM-CSF (Fig. 16 D). Lymph node cells and splenocytes restimulated from $T$ and $B$ cell-injected mice neither showed an increased sensitivity for the antigen, which would be reflected by an increased cytokine amount at a lower antigen concentration, nor a higher cytokine 
production than restimulated cells from $\mathrm{T}$ cell-injected mice. From that we conclude, that MOG-specific B cells did not accelerate the onset of EAE by influencing $T$ cell differentiation before onset of clinical EAE.
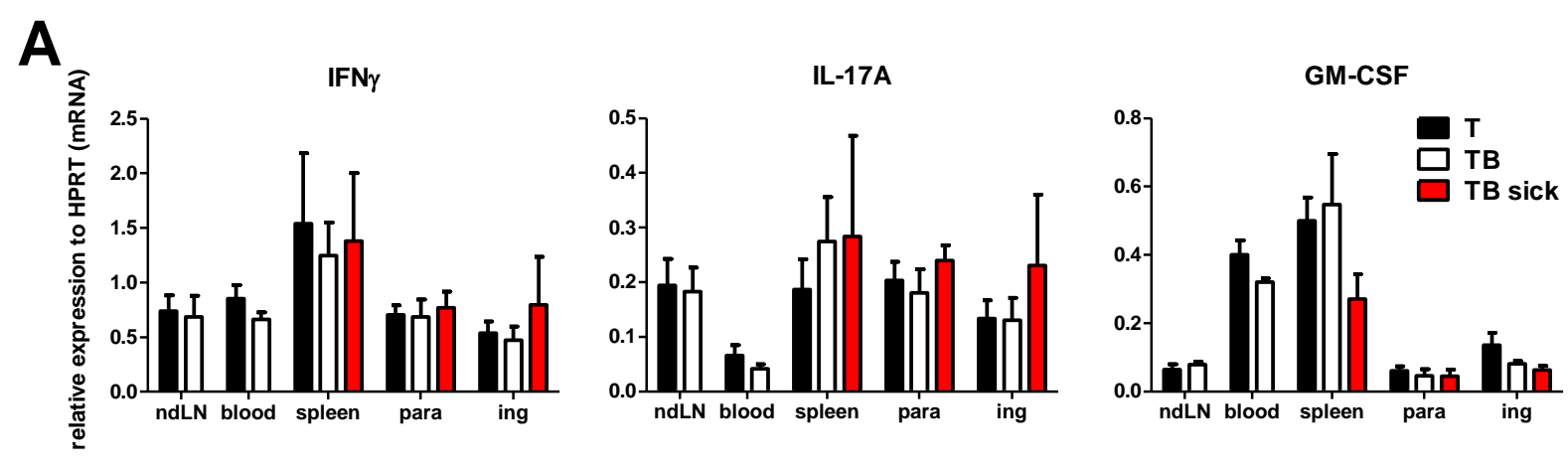

B
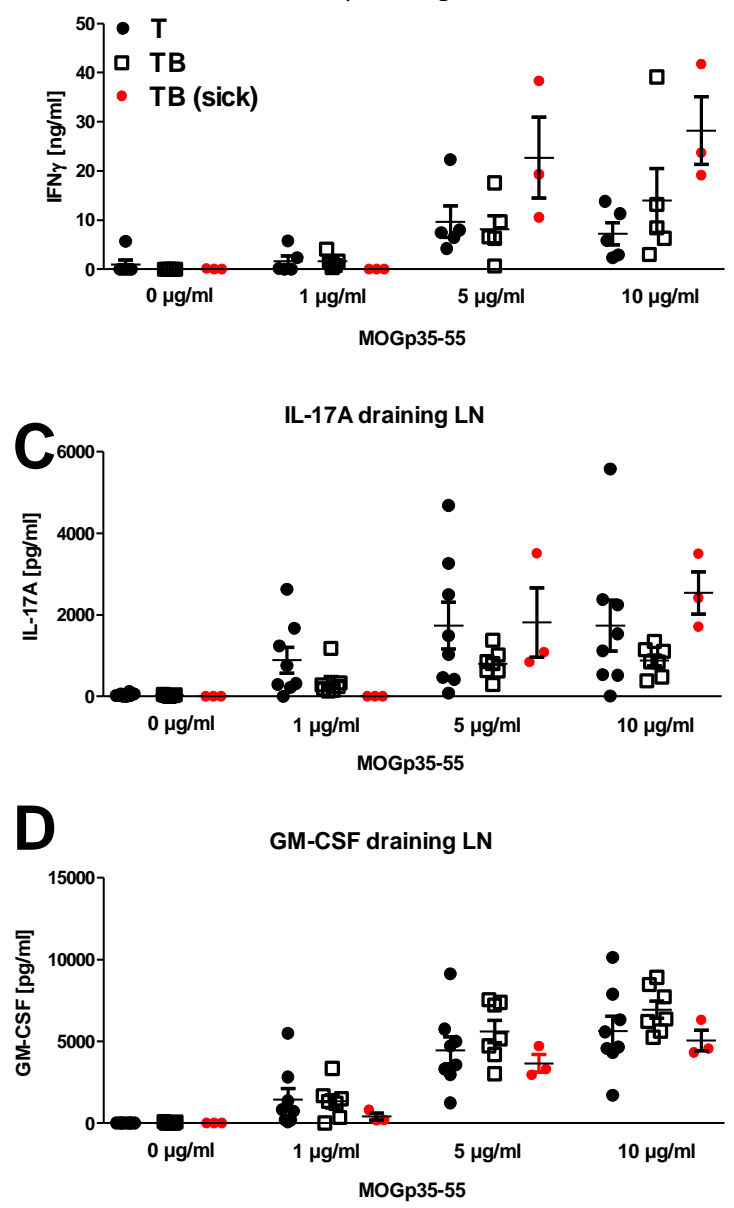


GM-CSF spleen

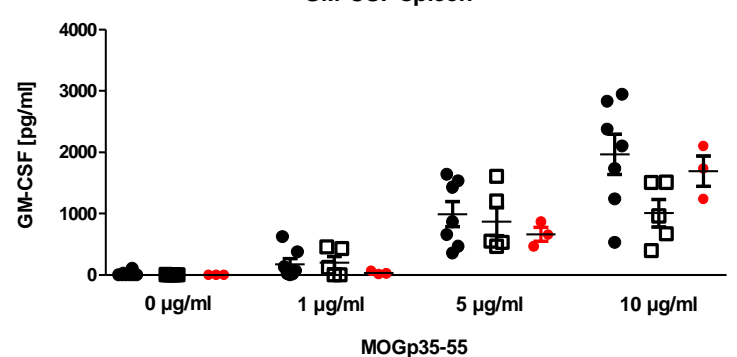

Figure 16: MOG-specific $B$ cells do not trigger $T$ cell differentiation before onset of EAE. (A) Cytokine expression (mRNA) of sorted RFP ${ }^{+}$cells isolated from blood and peripheral lymphoid organs of healthy $T$ cell (black bars) or $T$ and $B$ cell (white bar) or sick (Score 4) T and B cell (red bars) injected mice 9 days p.i. Data represents $n=6$ for $T$ and TB out of three independent experiments and $n=3$ for TB sick (B-D) total draining lymph node cells (left) or splenocytes (right) were taken from respective animals (healthy T inj.: black circle, healthy TB inj: white quad, sick TB inj.: red circle) $9 \mathrm{~d}$ p.i. and restimulated in vitro with increasing $\mathrm{MOG}_{\mathrm{p} 35-55}$ concentrations for $72 \mathrm{~h}$. Supernatants were collected for (B) IFN $\gamma$, (C) IL-17A or (D) GM-CSF analysis by ELISA. Each symbol represents an individual mouse out of three independent experiments, with the bar indicating the mean \pm SEM. 


\subsubsection{MOG-specific $B$ cells promote the earlier infiltration of $T$ cells into the}

\section{CNS}

We know from our clinical observations, that B cells accelerate the disease onset resulting in first clinical signs about 9.5 days post immunization (Table 4). Therefore, we wondered why the animals which received MOG-specific $T$ and $B$ cells develop EAE earlier than mice which just received MOG-specific $T$ cells. There are two main possible mechanisms known which can account for an earlier disease onset. On the one hand, it is possible that $B$ cells promote $T$ cell infiltration into the spinal cord and thus, lead to an earlier disease initiation. On the other hand, it was reported that rather the activation status than the number of infiltrating $T$ cells determine the clinical outcome, as antigen-unspecific T cells can infiltrate the CNS tissue in equal numbers than myelin-specific $T$ cells, but due to the failed reactivation, they are not able to induce clinical symptoms [115]. Therefore, it is possible that $B$ cells influence $T$ cell activation during the reactivation phase within the CNS. As B cells are the most prevalent MHC-II+ cell type within the naïve CNS, they cannot only act as APCs in the periphery, but it is suggested that they play a role in the initial steps of $T$ cell reactivation and thus lead to the recruitment of further cells into the CNS [154].

To investigate whether an earlier $\mathrm{T}$ cell infiltration accounts for the earlier onset, we determined the number of spinal cord infiltrated RFP ${ }^{+}$cells 8.5 to 9 days post immunization of animals either injected with $T$ cells and animals injected with $T$ and $B$ cells. To really figure out the situation before disease onset, we did not include animals with clinical symptoms in our analysis. To isolate the initial infiltrated T cells, which were located within the perivascular spaces, we did not implement the percoll gradient method, because this is more suitable for lymphocytes located within the CNS parenchyma. Instead, we adapted the spinal cord microvessel endothelial cell isolation method for that purpose. 




Figure 17: MOG-specific B cells promote the infiltration of MOG-specific T cells into the CNS. Number of RFP' cells isolated from spinal cord 9 days p.i.. Each symbol represents an individual mouse, with the bar indicating the mean \pm SEM. ( $n=9$ for $T$ and 8 for TB), Significant differences between means are indicated: ${ }^{*} p<$ 0.05 , unpaired students $t$ test

Most of the animals receiving $T$ and $B$ cells, analyzed shortly before the onset of clinical EAE, had a higher number of infiltrated $T$ cells in contrast to animals who just received MOG-specific $T$ cells (Fig. 17). To further exclude a difference in the reactivation status of infiltrated cells, we sorted the RFP ${ }^{+} \mathrm{T}$ cells of both, $\mathrm{T}$ cellinjected and $T$ and $B$ cell-injected-mice and determined the expression of IFN $\gamma$, IL$17 A$ and GM-CSF on mRNA level by RT-PCR. Here, it is important to mention, that we sorted the initial infiltrates of $T$ and $B$ cell-injected mice 9 days post immunization and of $T$ cell-injected mice 11-12 days post immunization to ensure the comparison of comparable cell numbers. The initial infiltrates of mice receiving MOG-specific $T$ cells alone and of those, who received both cell types, displayed comparable mRNA expression for IFN $\gamma$, IL-17A and GM-CSF (Fig. 18 A). Moreover, when we transferred GFP $^{+}$MOG-specific $B$ cells, we never detected them within the spinal cord of healthy animals 9 days p.i. (Fig. $18 \mathrm{~B}$ left) although they were present within the periphery (spleen). And even in sick animals, the presence of infiltrated $\mathrm{GFP}^{+} \mathrm{B}$ cells was very rare (Fig. $18 \mathrm{~B}$ right). From these observations we conclude that MOG-specific $\mathrm{B}$ cells promote the infiltration of $T$ cells and are not crucial for the reactivation step within the spinal cord in our experimental setting. Furthermore, we can exclude a critical role for $B$ cells in amplifying peripheral total $\mathrm{T}$ cell numbers or increasing the frequency of activated or differentiated $T$ cells. Therefore, we were interested whether $B$ cells influence $T$ cell migration in our experimental setting. 
A
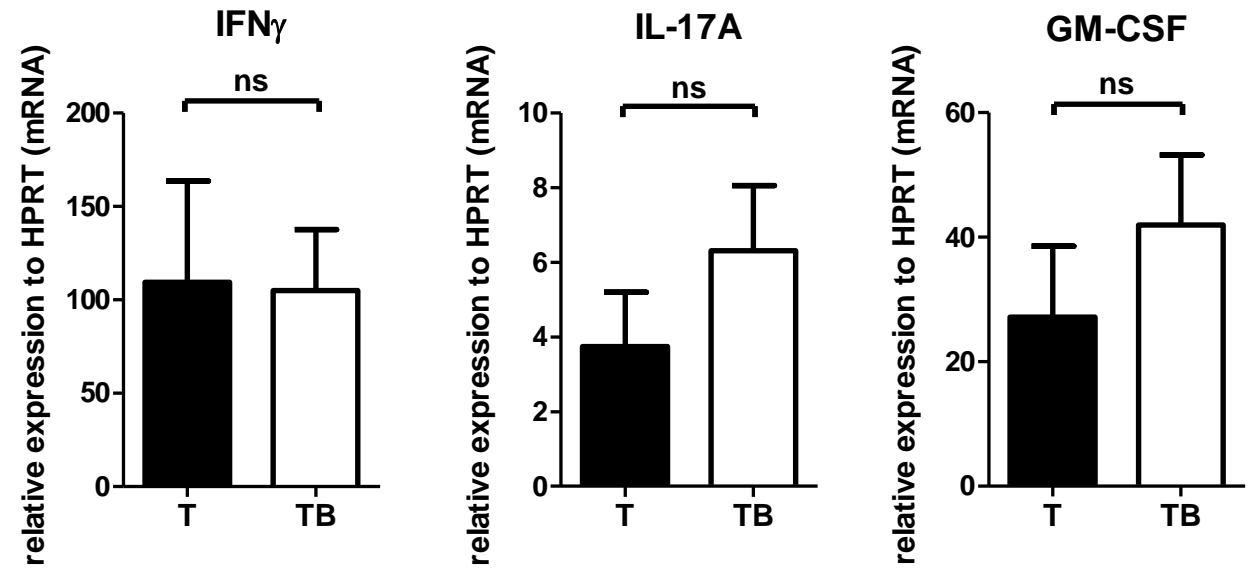

B

healthy


sick

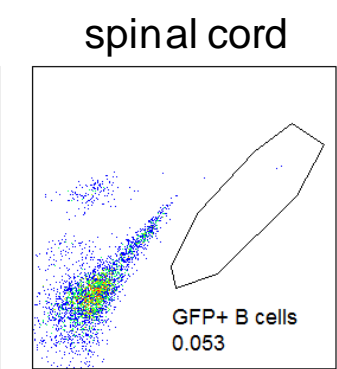

Figure 18: No difference in activation status of $\mathrm{RFP}^{+}$initial infiltrated $\mathrm{T}$ cells. (A) Cytokine expression (mRNA) of sorted RFP ${ }^{+}$cells isolated from spinal cord 11-12 days p.i. for $\mathrm{T}$ group and 9 days p.i. for TB group. (B) Frequency of GFP ${ }^{+} B$ cells in spleen and spinal cord of an healthy animal (left) and a sick animal (Score 4,5) (right) $9 \mathrm{~d}$ p.i.. Data in A represent three independent experiments with each 2 animals per group. Data are depicted as mean \pm SEM. Non-significant differences are depicted: ns $p>0.05$, unpaired students $t$ test 


\subsubsection{Effect of MOG-specific B cells on T cell migration into the CNS}

Our previous results led us to suggest that the accelerated onset in the presence of MOG-specific B cells is rather due to an increased T cell infiltration (Fig. 17) than to an increased reactivation (Fig. 18). Therefore, we focused on mechanisms required for T cell migration.

\subsubsection{MOG-specific T cells show an enhanced expression of CCR6 and even pronounced expression of CXCR4 in the presence of MOG-specific B cells}

The interaction of chemokines and their respective receptors, expressed by T cells, is a critical step for $T$ cell migration from the blood into various tissues. In the context of MS and EAE, several chemokine receptors have been found to be crucial for the infiltration into the CNS. Upon inflammation, TH1 cells upregulate the chemokine receptors CCR5 and CXCR3 and they were shown to be required for T cell migration into the CNS [118-122], whereas TH17 cells infiltrate the CNS in a CCR6-dependent manner [135]. Also T cells expressing homeostatic chemokine receptors like CXCR4 and CCR7 have been shown to be located within MS lesions, also suggesting a role for these receptors in CNS infiltration $[120,179]$. Although it is not described yet for $B$ cells, it is possible that the antigen-specific interaction of $T$ and $B$ cells lead to a kind of $T$ cell imprinting. This is for example described for the interaction of dendritic cells from the payers patch with $T$ cells resulting in an upregulation of integrins and chemokine receptors required for their homing into the gut [180]. To investigate whether the MOG-specific interaction of $T$ and $B$ cells results in a different chemokine receptor regulation than of $T$ cells injected without $B$ cell supplementation, we determined the chemokine receptor expression, precisely CCR7, CXCR3, CXCR4, CCR5 and CCR6 on mRNA level of initial CNS-infiltrated T cells. Like described in chapter 3.3.3., we sorted the initial RFP ${ }^{+} T$ cells of $T$ and $B$ cell-injected animals $9 d$ p.i. and these of $T$ cell-injected animals 11-12d p.i. according to their RFPexpression. 

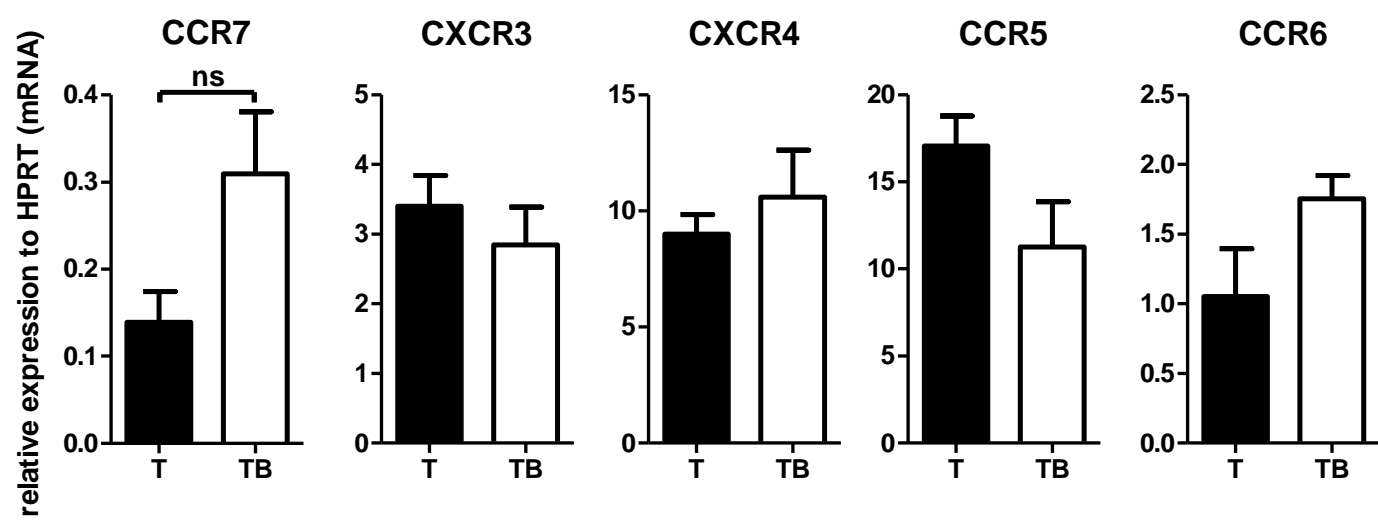

Figure 19: Initial infiltrated T cells utilize the same chemokine receptor for CNS infiltration. Chemokine receptor expression (mRNA) of sorted $\mathrm{RFP}^{+}$cells isolated from spinal cord 11-12 days p.i. for $T$ group and 9 days p.i. for TB group. Data represent three independent experiments with each 2 animals per group and are depicted as mean \pm SEM. Non-significant differences between means are indicated: ns $\mathrm{p}>0.05$, unpaired students $t$ test

We could not detect a significant difference of the chemokine receptor expression by the initial infiltrates of $\mathrm{T}$ cell-injected mice (Fig. 19 black bars) and $\mathrm{T}$ and $\mathrm{B}$ cellinjected mice (Fig. 19 white bars). However, there was a tendency that $T$ cells from $T$ and B cell-injected mice showed a higher CCR6 (TH17 cells) and CCR7 expression (although statistically not significant), in contrast to $\mathrm{T}$ cell-injected alone, which showed a higher expression of CCR5 (TH1 cells). This is in line with the cytokine expression of the initial infiltrated T cells (Fig. 18) as the T cells from $T$ and $B$ cellinjected animals showed a higher IL-17A and GM-CSF expression (but not statistically different). We were interested whether this observation could be reflected by $\mathrm{T}$ cells from the periphery. For this purpose, we sorted the RFP ${ }^{+}$MOG-specific $\mathrm{T}$ cells either from $\mathrm{T}$ cell-injected or from $\mathrm{T}$ and $\mathrm{B}$ cell-injected mice from peripheral lymphoid organs and from blood $9 \mathrm{~d}$ p.i. and performed chemokine receptor expression analysis by RT-PCR. In contrast to the initially infiltrated T cells from $T$ and $B$ cell-injected mice, $T$ cells sorted from the periphery of the same group did not reveal an increased CCR7 expression, but a slight increase in CCR6 expression of T cells isolated from the spleen (Fig. 20). As CCR6 ${ }^{+} \mathrm{TH} 17$ cells are known to be crucial for the initiation of $E A E$, it is likely that MOG-specific $B$ cells promote $E A E$ development by triggering either CCR6 upregulation or amplification of $\mathrm{CCR}^{+} \mathrm{T}$ cells. But $\mathrm{CCR}^{+} \mathrm{T}$ cells infiltrate the CNS through blood-cerebrospinal fluid barrier and therefore it is questionable that we did not detect an increased CCR6-expression of $T$ cells isolated from the blood [135]. This observation has to be elaborated in detail to come to a definitive answer. Moreover, we could not detect a difference in 
CXCR3 and CCR5 expression of sorted RFP ${ }^{+}$MOG-specific T cells between the two groups (Fig. 20). However, RFP ${ }^{+} \mathrm{T}$ cells sorted from spleen, blood and draining lymph nodes of $T$ and $B$ cell-injected mice significantly upregulated CXCR4 (Fig. 20). As the role of CXCR4 in lymphocyte migration into the CNS is controversial, this observation is difficult to interpret, especially because the initial infiltrated $T$ cells did not show an increased CXCR4 expression (Fig. 19).
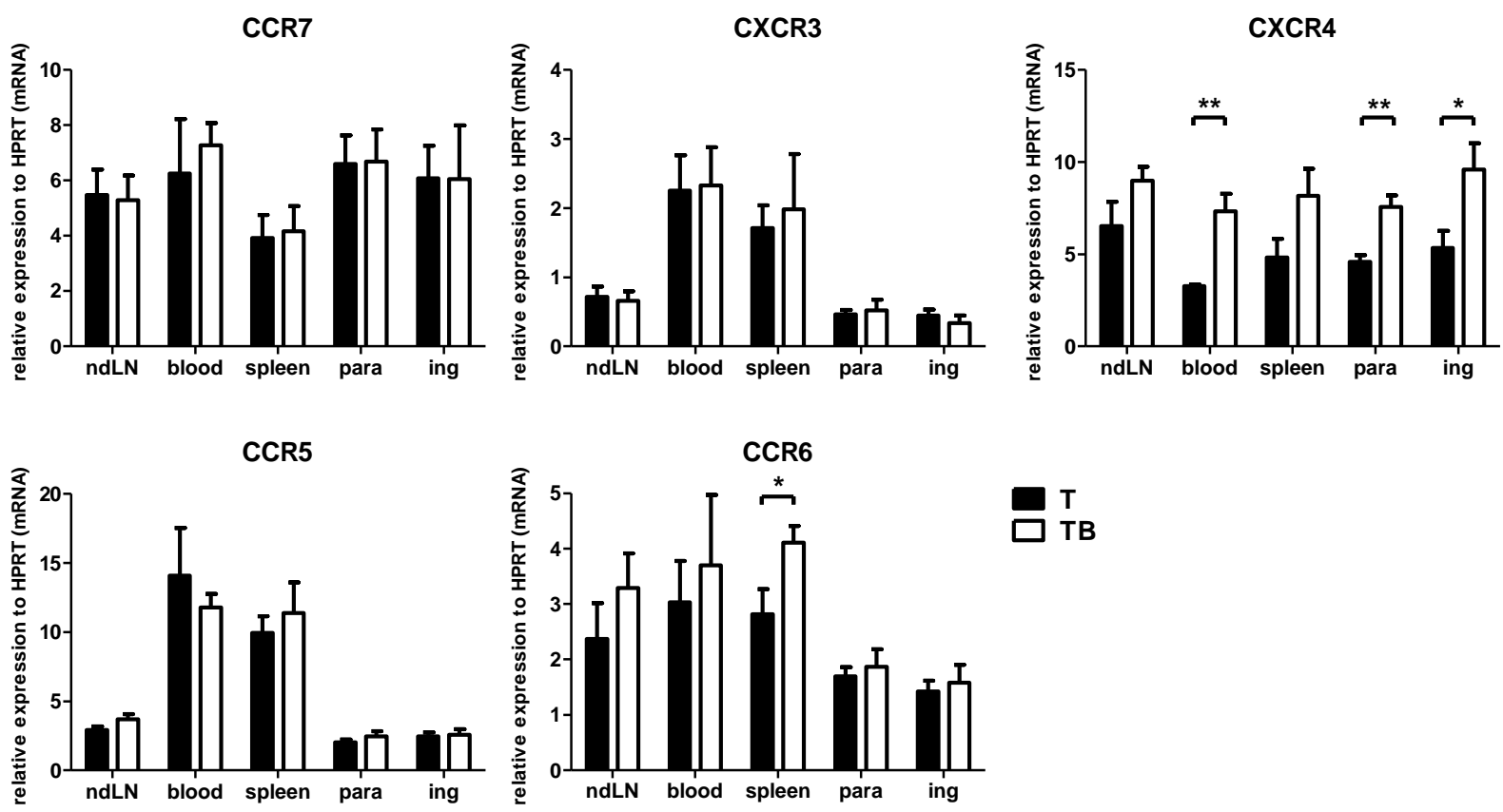

Figure 20: MOG-specific B cells influence CXCR4 expression of T cells but have no effect on the other chemokine receptors. Chemokine receptor expression (mRNA) of sorted $\mathrm{RFP}^{+} \mathrm{CD}^{+} \mathrm{T}$ cells from blood, non-draining lymph nodes, spleen and draining lymph nodes $9 \mathrm{~d}$ p.i.. Data represent three independent experiments with each 2 animals per group. Significant differences are indicated: ${ }^{*} p<0.05$ and ${ }^{* *} p<0.01$, unpaired students $t$ test, shown are means \pm SEM. 


\subsubsection{MOG-specific $B$ cells do not influence adhesion molecule expression of} T cells

Adhesion molecules like VLA-4 and LFA-1 expressed by T cells have been shown to be crucial for the initial interaction of $T$ cells with endothelial cells of CNS microvessels as well as for their transmigration $[99,106]$. Therefore, we investigated whether MOG-specific $B$ cells trigger the upregulation of either LFA-1 or VLA-4 on T cells or whether they expand LFA-1 $1^{+}$or VLA-4 $4^{+}$MOG-specific $T$ cells in our experimental setting. For expression analysis we sorted $\mathrm{RFP}^{+} \mathrm{CD} 4^{+} \mathrm{T}$ cells $9 \mathrm{~d}$ p.i. from either $T$ cell-injected mice (Fig. $21 \mathrm{~A}$ black bars) or $\mathrm{T}$ and $\mathrm{B}$ cells-injected mice (Fig. $21 \mathrm{~A}$ white bars) and determined integrin alpha 4 (alpha subunit of VLA-4) or integrin alpha $L$ (alpha subunit of LFA-1) mRNA expression by RT-PCR. When we compared the mRNA expression of $T$ cells sorted from lymph nodes, spleen and blood, we could neither detect a significant difference in integrin alpha 4 nor in integrin alpha $L$ expression between the two groups (Fig. $21 \mathrm{~A}$ ). As it is possible that the expression is not regulated on mRNA level, but that $B$ cells rather amplify VLA-4or LFA-1-positive T cells, we determined the number of positive cells within the blood and spleen between day 7 and day 9 p.i. (Fig. $21 \mathrm{~B}+\mathrm{C}$ ). The number of VLA$4^{+} \mathrm{RFP}^{+} \mathrm{CD} 4^{+} \mathrm{T}$ cells increased over time in the blood as well as in the spleen (Fig. 21 $B)$, but there was no difference in the $T$ cell number of mice injected with $T$ cells and of these injected with $T$ and $B$ cells. In accordance to that, we could not detect any difference in $\mathrm{LFA}-1^{+} \mathrm{RFP}^{+} \mathrm{CD} 4^{+} \mathrm{T}$ cell number (Fig. $21 \mathrm{C}$ ). We therefore conclude that MOG-specific $B$ cells do not influence $T$ cell migration into the CNS by affecting their expression of chemokine receptors or integrins. 
A

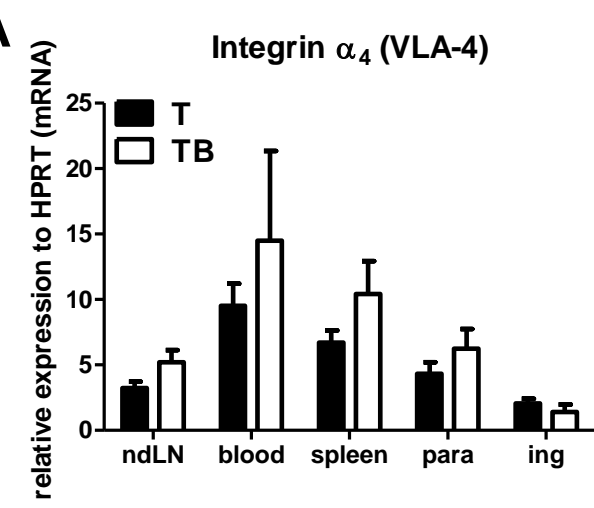

B

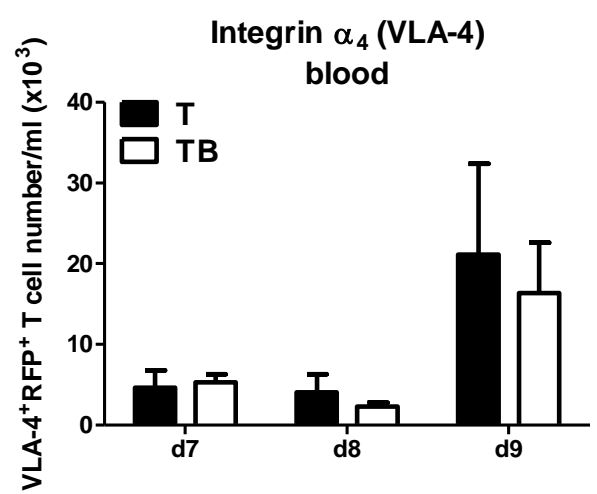

C

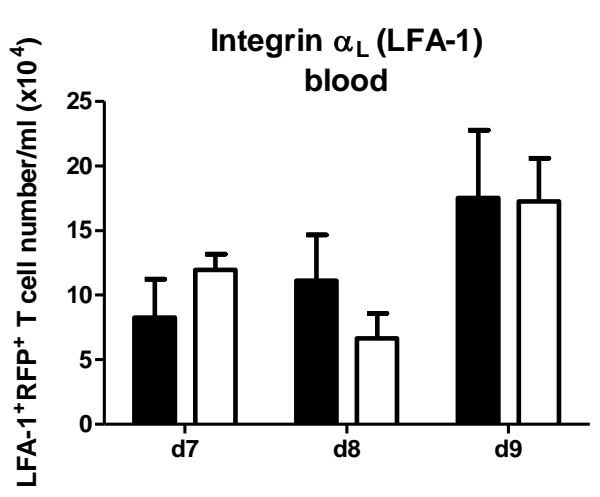

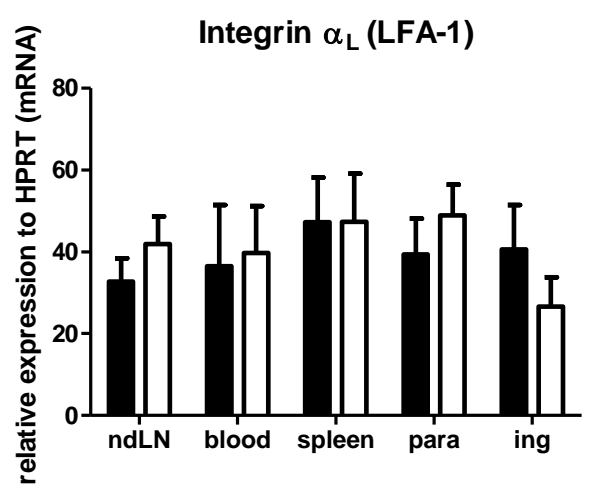
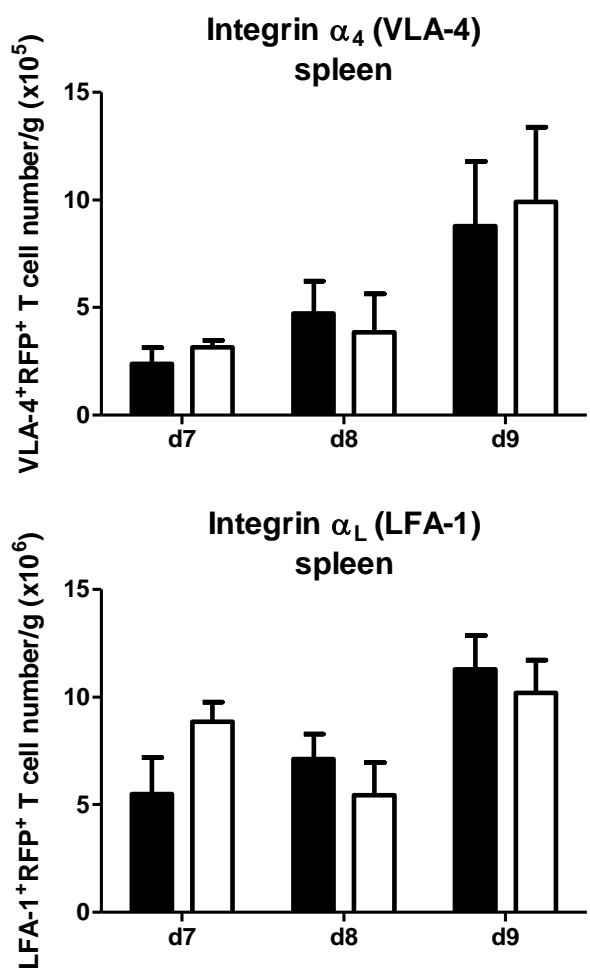

Figure 21: MOG-specific B cells do not influence integrin expression of $T$ cells. (A) Integrin expression (mRNA) of sorted $\mathrm{RFP}^{+} \mathrm{CD}^{+} \mathrm{T}$ cells from blood, non-draining lymph nodes, spleen and draining lymph nodes $9 \mathrm{~d}$ p.i. (B) Quantification of VLA-4 ${ }^{+} \mathrm{RFP}^{+} \mathrm{CD4} 4^{+} \mathrm{T}$ cells from the blood (left) and from the spleen (right) with flow cytometry on d7-d9 p.i. (C) Quantification of LFA-1 ${ }^{+}$ $\mathrm{RFP}^{+} \mathrm{CD} 4^{+} \mathrm{T}$ cells from the blood (left) and from the spleen (right) with flow cytometry on d7-d9 p.i. Data in A represent three independent experiments with each 2 animals per group. Data in $B+C$ represent two independent experiments with at least 2 animals per group. Shown are mean \pm SEM 


\subsection{MOG-specific B cells do not influence mobilization of myeloid cells}

As we could not detect any differences in the T cell phenotype which would allow their earlier infiltration into the CNS, we determined whether MOG-specific B cells might influence other cell types responsible for the accelerated disease onset. Several publications suggest a crucial role for monocytes in disease initiation. $E A E$ studies with $\mathrm{CCR}^{-/}$mice, the chemokine receptor predominantly expressed by inflammatory monocytes, reveal that the impaired CNS infiltration of monocytes is accompanied by a reduced number of infiltrating $T$ cells $[165,167]$. In addition, the increase of $\mathrm{Ly}_{6 \mathrm{C}} \mathrm{C}^{+}$monocyte number in the blood was associated with an earlier $\mathrm{EAE}$ disease onset [181]. In concordance, it was shown that $B$ cells can influence monocyte mobilization in acute myocardial infarction [182]. Therefore, we were interested whether monocyte mobilization is affected in our experimental setting. Monocytes mainly appear in the blood as two populations: inflammatory monocytes

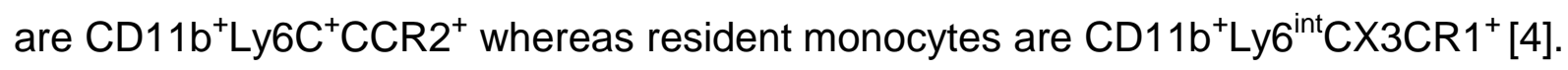
As these populations originate from a common precursor, the simultaneous expression of CCR2 and CX3CR1 is not unusual. To investigate whether the frequency of monocyte subpopulations is altered between $T$ cell-injected and $T$ and $B$ cell-injected mice before EAE onset, we determined the number of the different monocyte populations in the blood, the spleen and the spinal cord on day 7 and 8 p.i. by flow cytometry. Different monocyte subpopulations were gated like depicted in Fig. 22 A. Seven days p.i. we detected a slight increase of total CD11 b ${ }^{+} L^{2} 6 C^{\text {hi }}$ cells,

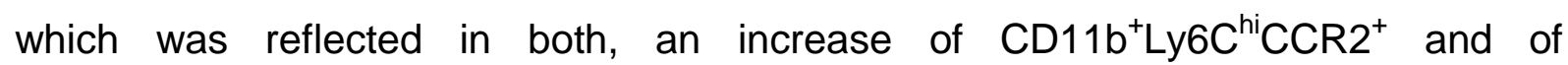
CD11 $\mathrm{b}^{+}$Ly6 $\mathrm{C}^{\text {hi }} \mathrm{CCR} 2^{+} \mathrm{CX} 3 \mathrm{CR} 1^{+}$in the blood, but not in the spleen and spinal cord of $\mathrm{T}$ and B cell-injected mice (Fig. 22 B). One day later the picture completely changed with an increased number of $C D 11 b^{+} L y 6 C^{\text {hi }}$ monocyte subpopulations in the blood of T cell-injected mice (Fig. $22 \mathrm{C}$ left), but a tendency of an increased number of that subpopulations in the spinal cord of $T$ and $B$ cell-injected mice (Fig. $22 \mathrm{C}$ right). However, as we could not detect a unique increase of the CD11 $\mathrm{b}^{+} L y 6 \mathrm{C}^{\text {hi }}$ monocyte subpopulations in the blood as described in the literature, we conclude that $B$ cells do not influence monocyte mobilization in our experimental setting. 
A


B


Figure 22: MOG-specific $B$ cells do not influence monocyte mobilization. (A) Flow cytometry gating strategy for different monocyte subpopulations. (B-C) different monocyte subpopulations (all gated on CD11b) were quantified in blood, spleen and spinal cord of T cell-injected (black bars) and T and B cell injected mice (white bars) (B) $7 \mathrm{~d}$ p.i. and (C) $8 \mathrm{~d}$ p.i.. Data represents 2 independent experiments with each 2 animals per group. Shown are mean \pm SEM 


\subsection{MOG-specific B cells do not mediate upregulation of adhesion molecules and chemokines by spinal cord microvessel endothelial cells}

During EAE, endothelial cells of spinal cord microvessels have been shown to upregulate the expression of several chemokines and adhesion molecules required for lymphocyte attraction, adhesion and transmigration [112, 125, 126, 129]. Therefore, it is possible that B cells trigger chemokine or adhesion molecule upregulation, for example through soluble factors like cytokines, and thus promote lymphocyte infiltration into the CNS. In line with this, it was shown that B cells are the major source of IL-6 in secondary lymphoid tissues and that absence of IL-6 during EAE results in an impaired upregulation of ICAM-1 and VCAM-1 of CNS endothelial cells [183]. To investigate whether the presence of MOG-specific $B$ cells promote the upregulation of adhesion molecules and chemokines before onset of EAE, we isolated spinal cord microvessels and sorted the endothelial cells according to their CD31 (PECAM-1) expression of T cell-injected and T and B cell-injected mice 8 days p.i.. We quantified their ICAM-1, VCAM-1, ALCAM, CXCL10, CXCL12, CCL5 mRNA expression by RT-PCR. To ensure that endothelial cells were isolated from mice at the same preclinical stage, we additionally determined the number of infiltrated RFP ${ }^{+}$ cells and only endothelial cells with comparable low numbers of infiltrated cells ( $\sim 30$ 60 cells) were used for gene expression analysis. Otherwise, we could not exclude that the presence of already infiltrated cells and their ongoing reactivation contributed to an increased expression of adhesion molecules and chemokines. 
A

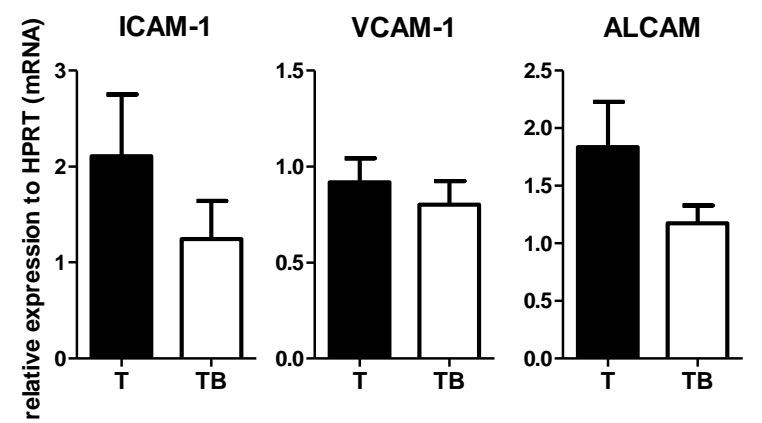

B

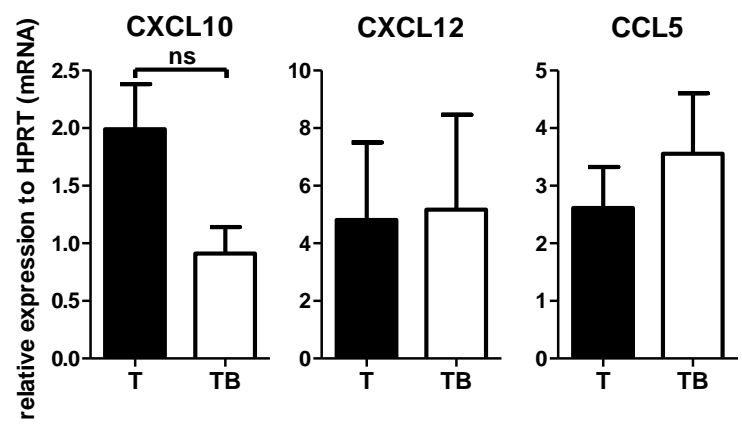

Figure 23: MOG-specific $B$ cells do not trigger the upregulation of adhesion molecules and chemokines by spinal cord microvessel endothelial cells. Spinal cord microvessels were isolated from T cell-injected and T and B cell-injected OT-II mice with similar CNS infiltrated RFP ${ }^{+}$cell numbers and endothelial cells were sorted according to their CD31 expression 8d p.i.. (A) Adhesion molecules (ICAM-1, VCAM-1, ALCAM) and (B) chemokine (CXCL10, CXCL12, CCL5) (mRNA) expression analysis was performed by RT-PCR. Data represent 2 independent experiments with 4 animals per group. Data are depicted as mean \pm SEM. Non-significant differences between means are indicated: $n s p>0.05$, unpaired students $t$ test

Although we observed an increased number of CNS infiltrated T cells upon additional transfer of MOG-specific $B$ cells, these cells did not trigger the upregulation of adhesion molecules (Fig. 23 A) or chemokines (Fig. 23 B) by spinal cord microvessel endothelial cells. On the contrary, endothelial cells isolated from $T$ cell-injected mice even showed an enhanced expression of ALCAM and of CXCL10 on mRNA level in contrast to $T$ and $B$ cell-injected mice. Therefore, it is unlikely that $B$ cells promote $T$ cell infiltration into the CNS by influencing microvessel endothelial cells. 


\subsection{The antigen-specific interaction of $T$ and $B$ cells determine the accelerated disease onset}

As we could not detect any obvious or detectable difference in the T cell phenotype or an effect of B cells on other cell types like monocytes or spinal cord microvessel endothelial cells, we wanted to search for other mechanisms by which B cells contribute to the accelerated disease onset. We were interested whether the presence of activated, but not MOG-specific B cells is sufficient to accelerate the disease onset or whether the antigen-specific interaction of $T$ and $B$ cells determines the effect on EAE onset. For that purpose, we supplemented OT-II mice with different combinations of $\mathrm{T}$ and $\mathrm{B}$ cells and immunized them with NP-OVAL and MOG $\mathrm{p}_{\mathrm{p} 5-55}$ (Table 5) and monitored their clinical outcome.

Table 5: Overview of clinical experiment

\begin{tabular}{c|c} 
Cell transfer & Antigen \\
\hline MOG-specific T cells & NP-OVAL + MOG $_{p 35-55}$ \\
\hline MOG-specific T and B cells & NP-OVAL + MOG $_{p 35-55}$ \\
\hline MOG-specific T cells and & NP-OVAL + MOG $_{p 35-55}$ \\
NP-specific B cells & \\
\hline
\end{tabular}

To investigate whether the presence of activated, but not MOG-specific B cells is sufficient to accelerate the onset of EAE, we transferred NP-specific $B$ cells together with MOG-specific T cells into OT-II hosts and immunized them with a combination of NP-OVAL and $M O G_{p 35-55}$. We know from in vitro studies that NP-specific B cells become activated by the NP-OVAL in the presence of OVA-specific $T$ cells (endogenous cells) (Fig $6 \mathrm{~B}+\mathrm{C}$ right). We compared the clinical outcome to either MOG-specific T cell-injected mice or to MOG-specific T and B cell-injected mice, both immunized with NP-OVAL and $\mathrm{MOG}_{\mathrm{p} 35-55}$, but we did not expect an effect of NPOVAL on the disease development in these groups. To proof that the NP-specific B cells became activated in vivo, we determined their $\lg G$ production against OVA, rrMOG and NP-OVAL and could show that they specifically produced anti-NP-OVAL lgG (Fig. 24 C). 
A

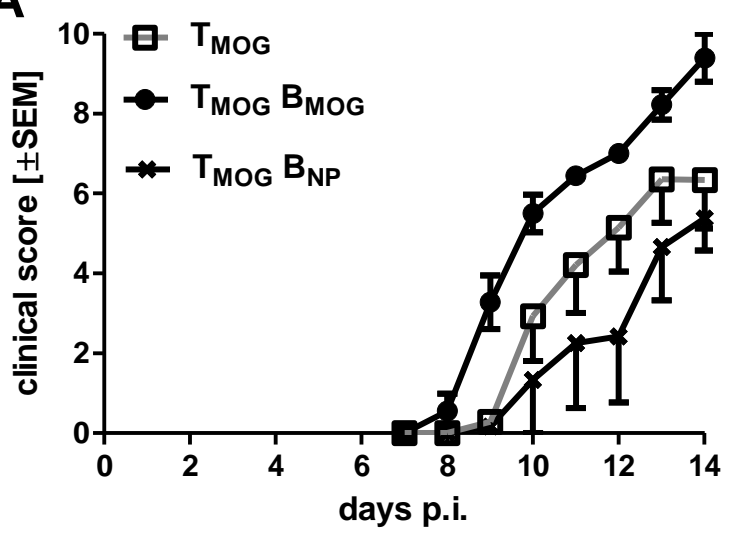

C



B

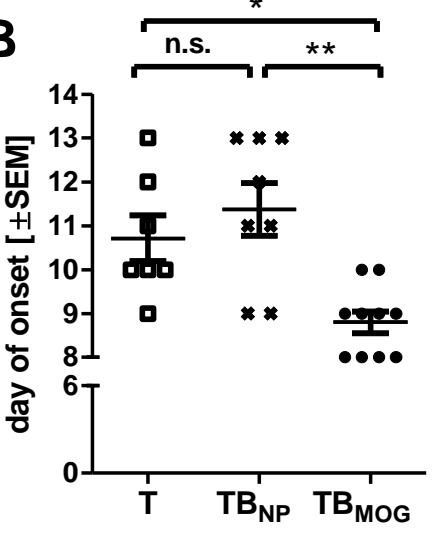

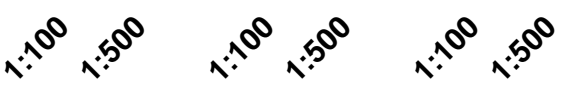

sera dilution

Figure 24: The antigen-specific interaction of $T$ and $B$ cells determine the clinical outcome of EAE. OT-II mice were injected either with MOG-specific T cells, MOG-specific T and B cells or MOG-specific T cells and NP-specific B cells followed by immunization with NP-OVAL and $M O G_{\text {p35-55. }}$ (A) EAE disease course of OT-II injected mice (B) Day of onset of each animal per T or $T_{B} B_{M O G}$ or TB $B_{N P}$ group. Shown are two pooled experiments with 9 animals per group. (C) Serum antiOVA, anti-rrMOG and anti-NP-OVAL IgG titer of MOG-specific T and NP-specific B cell-injected OT-II mice obtained $10-12 \mathrm{~d}$ p.i. from peripheral blood, pooled data from 5 animals. Data show mean \pm SEM. Significant differences between means are indicated: $n s p>0.05,{ }^{*} p<0.05{ }^{* *} p<0.01$, 1 way ANOVA

Whereas MOG-specific $T$ and $B$ cell-injected mice developed first clinical signs at about day $8.8 \pm 0.24$, the presence of in vivo activated NP-specific B cells had no effect on the onset compared to MOG-specific T cell-injected mice (TB $\mathrm{B}_{\mathrm{NP}}$ : day 11.38 \pm 0.59 , $\mathrm{T}$ : day $10.71 \pm 0.52$ ) (Fig. $24 \mathrm{~A}+\mathrm{B}$ ). We therefore assume, that the antigenspecific interaction of MOG-specific $T$ and $B$ cells rather determine the clinical outcome than the presence of activated MOG-unspecific B cells. 


\subsection{MHC-II deficiency of MOG-specific B cells do not exclusively affect their antigen-presenting capacity}

We know from the previous clinical experiment that activated, so-called "bystander" B cells had no effect on the onset of EAE. Therefore, we were interested about the mechanism which is based on the antigen-specific interaction of $T$ and $B$ cells. To interfere with this mechanism, we crossed Th mice to $\mathrm{MHC}-\mathrm{II}^{-/-}$mice to generate MHC-II ${ }^{-/-}$MOG-specific $B$ cells with the final intention to use these $B$ cells for adoptive transfer experiments. To ensure that $\mathrm{MHC}-\mathrm{II}^{-/-} \mathrm{MOG}$-specific $\mathrm{B}$ cells were really impaired in their antigen-presenting capacity, we first tested them in an in vitro proliferation assay. We cultured CFSE-labeled MOG-specific T cells either with MOG-specific B cells or with MHC- $\mathrm{II}^{-/-}$MOG-specific B cells in the presence of increasing concentrations of either $\mathrm{MOG}_{\mathrm{p} 35-55}$ or rrMOG and assessed MOG-specific $\mathrm{T}$ cell proliferation after $72 \mathrm{~h}$. In addition, we analyzed in vitro B cell proliferation to investigate whether the defective antigen-presentation also impaired B cell function. We observed that $\mathrm{MHC}-\mathrm{II}^{-/-} \mathrm{B}$ cells were impaired in triggering $\mathrm{T}$ cell proliferation (Fig. 25 A). Moreover, MHC-II deficiency resulted in defective B cell response as their antigen-specific proliferation was also impaired (Fig. 25 B). As MHC-Il deficiency not only resulted in their impaired antigen-presenting capacity, but also affected their cellular behavior, we wondered whether this also had consequences in vivo. A recent publication observed that MHC-II deficiency of B cells was accompanied by an impaired secretion of antibodies [139]. Therefore, we injected MOG-specific B cells or MHC-II ${ }^{-/-}$MOG-specific B cells into NP mice and immunized them with a high dose of rrMOG $(40 \mu \mathrm{g})$ and determined anti-rrMOG IgG levels $8 \mathrm{~d}$ and 18d p.i.. We used NP mice recipients for that analysis as they are devoid of endogenous antibody-secreting MOG-specific B cells (Fig. 25 C lower row PBS ctrl). We detected substantial amounts of anti-rrMOG IgGs in serum of MOG-specific B cell-injected NP mice even after $8 \mathrm{~d}$ p.i.. In contrast, MHC- $\mathrm{II}^{-/}$MOG-specific B cells did not show an antibody response $8 \mathrm{~d}$ p.i. or at later time points (18d p.i.), suggesting that the MHC-II deficiency not only affected their cellular-, but also their humoral immune response. Thus, we proposed that these $B$ cells were not suitable to investigate the exclusive impact of antigen presentation on the course of EAE. 
A


B

\section{B cell proliferation}
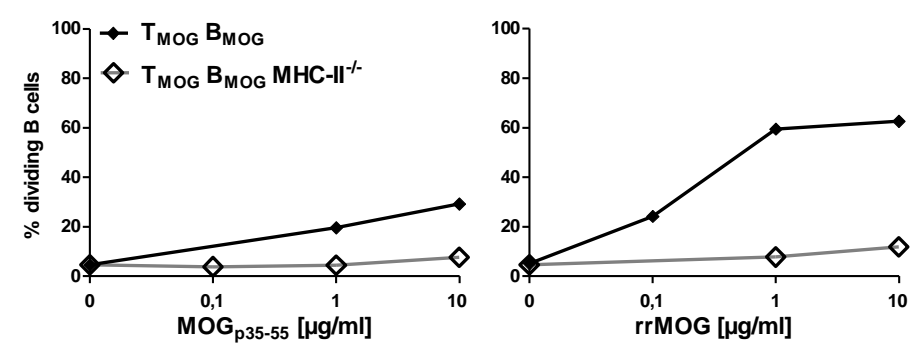


$\alpha-\operatorname{rrMOG} \lg G$ $18 d$ p.i.

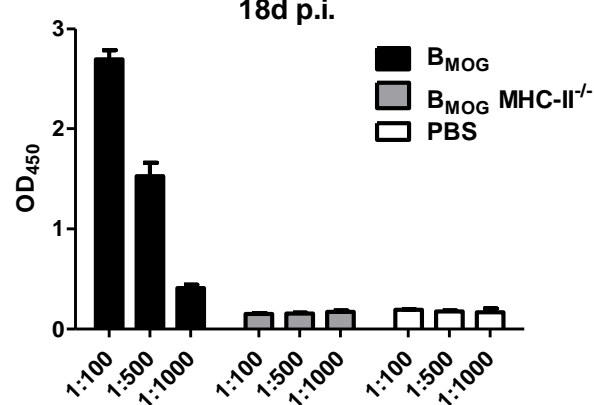

Figure 25: Ablation of MHC-II of $B$ cells results in impaired $B$ cell function.

(A+B) $1 \times 10^{6}$ MOG-specific T cells were co-cultured with either $2 \times 10^{6}$ MOG-specific B cells (black line) or MHC-II ${ }^{-1-}$ MOG-specific B cells (grey line) with increasing $\mathrm{MOG}_{\mathrm{p} 35-55}$ (left) or rrMOG (right) concentrations $(0 ; 0,1 ; 1 ; 10 \mu \mathrm{g} / \mathrm{ml})$ for $72 \mathrm{~h}$. (A) $\mathrm{T}$ cell and (B) B cell proliferation was determined by CFSE dilution. (C) MOG-specific B cells, MHC-II- MOG-specific B cells or PBS were i.v. injected into $\mathrm{NP}$ mice and immunized two days later with $40 \mu \mathrm{g}$ rrMOG. Sera were obtained $8 \mathrm{~d}$ p.i. (upper row) and 18d p.i. (lower row) and anti- anti-rrMOG IgG was quantified by ELISA, graphs are depicted as mean \pm SEM. 


\subsection{The role of antibodies in $M O G_{p 35-55}$ induced EAE}

\subsubsection{MOG-specific $B$ cells produce anti-rrMOG $\operatorname{Ig} G$ upon $M O G_{p 35-55}$ immunization}

As we have analyzed the phenotype of the MOG-specific $T$ cells in the previous experiments extensively during the activation phase and before the disease onset and could not detect any striking difference, we suppose that the disease-accelerated effect is mediated by the MOG-specific B cells. That assumption is supported by the observation that activated MOG-unspecific $B$ cells did not accelerate the disease onset (Fig. 24 A+B). Of course, MOG-specific $B$ cells and NP-specific $B$ cells differ in their antibody specificity. Although several publications suggest that anti-MOG antibodies are not produced after MOG ${ }_{\mathrm{p} 35-55}$ immunization [139, 151], we investigated whether this also holds true in our experimental setting. Because we know that NPspecific $B$ cell mice were devoid of endogenous antibody-secreting MOG-specific B cells, we used these mice as hosts to analyze whether the transferred MOG-specific $B$ cells were in general able to exhibit an humoral immune response after MOG peptide immunization. We injected MOG-specific B cells or PBS as control into NPhost mice and immunized them two days later with $\mathrm{MOG}_{\mathrm{p} 35-55}$, obtained blood $9 \mathrm{~d}$ p.i. and assessed the amount of anti-MOG $\mathrm{p} 35-55_{5} \lg \mathrm{M}$ and $\lg \mathrm{G}$ and of anti-rrMOG $\lg M$ and IgG by ELISA. 

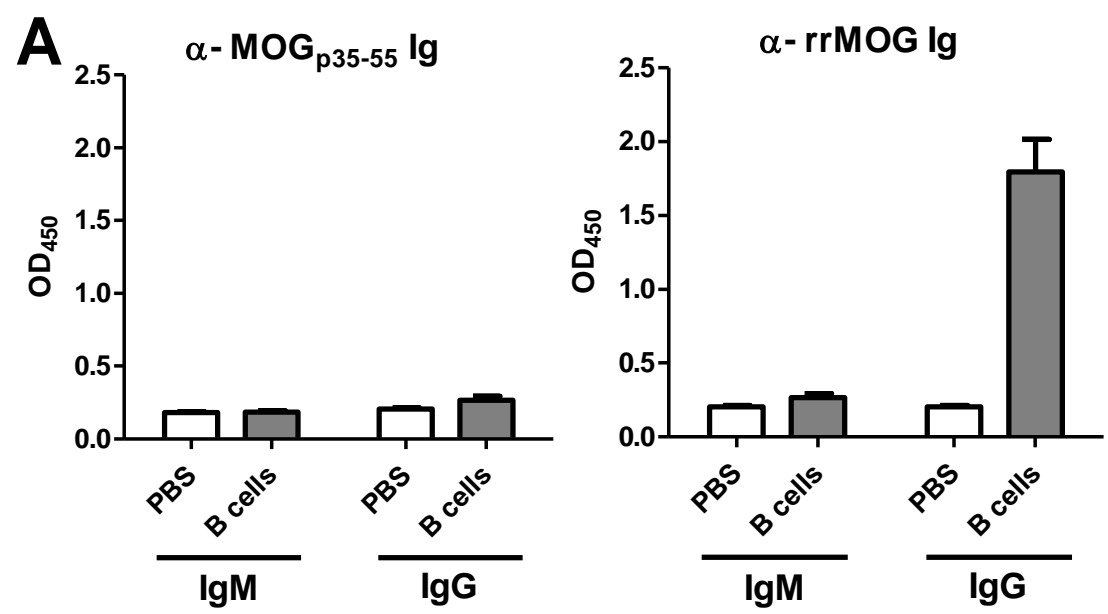

B
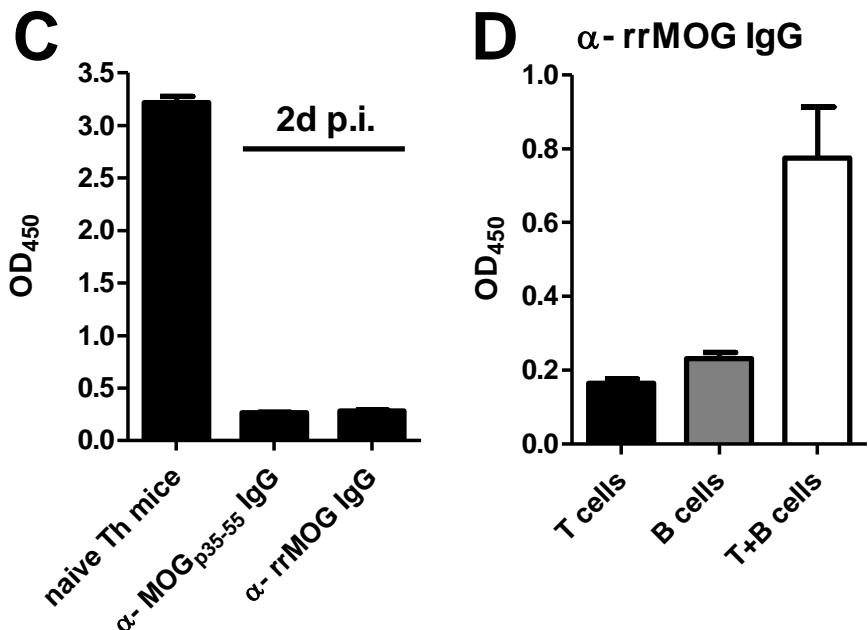

Figure 26: MOG-specific $B$ cells produce anti-rrMOG IgG upon $\mathrm{MOG}_{\mathrm{p} 35-55}$.

immunization. (A) MOG-specific B cells or PBS were i.v. injected into NP mice and immunized two days later with $\mathrm{MOG}_{\mathrm{p} 35-55}$. Sera were obtained $9 \mathrm{~d}$ p.i. and anti-MOG $\mathrm{p} 35-55_{5} \operatorname{lgM}$ and $\lg \mathrm{G}$ (left) and antirrMOG IgM and IgG (right) was quantified by ELISA. (B) MOG-specific B cells or PBS were i.v. injected into WT mice and immunized two days later with $M_{0} G_{p 35-55}$. Sera were obtained $9 d$ and $30 d$ p.i. and anti-rrMOG IgG was quantified by ELISA (C) Serum was obtained from naïve Th mice or MOG-specific B cell-injected WT mice 2d p.i. and anti-MOG ${ }_{\mathrm{p} 35-55} \lg \mathrm{g}$ and anti-rrMOG IgG (naïve Th mice) was quantified by ELISA. (D) MOG-specific T cells, MOG-specific B cells or MOG-specific T and $B$ cells were i.v. injected in OT-II, immunized with $M O G_{p 35-55}$ and serum was obtained 9-12d p.i.. AntirrMOG IgG was quantified by ELISA. Data in A and B represent 4 mice per group, in $C 2$ mice per group and in $D 6$ mice per group, shown are mean \pm SEM.

Transferred MOG-specific B cells did not secrete anti-MOG p35-55 $_{\text {IgM }}$ or IgG (Fig. 26 A, left) 9 days post immunization, which is not surprising because B cells are known to rather recognize structural epitopes than short amino acid sequences. However, although we immunized with MOG peptide, we detected anti-rrMOG IgG (Fig. 26 A right). As we could not detect any anti-rrMOG specific lgGs in PBS injected mice, we assumed that the transferred $B$ cells were the source of antibodies. When we repeated the experiment in WT mice, we observed that the transferred MOG-specific $B$ cells exhibited a stronger antibody response than the endogenous $B$ cells at an 
earlier time point (Fig. 26 B), but even endogenous B cells produced anti-rrMOG IgGs after MOG peptide immunization (30d p.i.). It is known that naïve Th mice, the donor of MOG-specific B cells, harbor a high titer of anti-rrMOG IgG (Fig. 26 C). Therefore, it is possible that the detected anti-rrMOG IgG are an artificial effect of transferring already differentiated antibody-secreting plasma blasts. However, upon transfer of MOG-specific B cells, they did not immediately ( $2 \mathrm{~d}$ p.i.) produce and secrete anti-rrMOG specific IgGs (Fig. $26 \mathrm{C}$ ). Thus, we assumed that the transferred MOG-specific $B$ cells became activated in vivo by immunization. To verify whether these observations also hold true in our experimental setup, we transferred either MOG-specific T cells, MOG-specific B cells or both together into OT-II mice, immunized them with $\mathrm{MOG}_{\mathrm{p} 35-55}$ two days later and determined anti-rrMOG IgG from sera obtained 9-12d p.i.. The transfer of MOG-specific $T$ cells did not trigger antiMOG IgG production of the endogenous $B$ cell pool (at least not until this early time point) and also MOG-specific $B$ cells did not secrete any anti-rrMOG IgG, presumably due to the absent antigen-specific T cell help (Fig. 26 D). However, the simultaneous transfer of MOG-specific $T$ and $B$ cells resulted in an increased anti-rrMOG IgG production (Fig. $26 \mathrm{D}$ ). Conclusively, although several publications suggest that $B$ cells do not exhibit an humoral immune response after MOG peptide immunization, we were indeed able to detect MOG-specific antibodies in our experimental setup. Moreover, the transfer of MOG-specific $T$ cells alone did not facilitate an humoral immune response against MOG of the endogenous $B$ cell pool leading to the assumption that the antibodies in theory can account for an accelerated disease onset in OT-II mice.

\subsubsection{Mice with XBP-1 deficient B cells lack anti-rrMOG specific Igs and show a delayed disease onset upon rrMOG immunization}

To investigate the role of antibodies in our experimental setting, we made use of a mouse system where $B$ cells are incapable to develop into antibody-secreting plasma cells. The transcription factor $\mathrm{X}$-box binding protein 1 (XBP-1) is required for the differentiation of $\mathrm{B}$ cells to plasma cells and XBP-1 deficient lymphoid chimeras are devoid of immunoglobulin of any isotype and of plasma cells [184]. To generate mice where XBP-1 deficiency is restricted to the $\mathrm{B}$ cell compartment, we crossed mb1Cre mice with XBP-1 $1^{\text {flffl }}$ mice resulting in Cre expression under the control of the $\mathrm{B}$ cell- 
specific mb1 promotor and subsequent specific deletion of XBP-1 [174]. Before crossing these mice to Th mice to obtain MOG-specific XBP-1-deficient B cells, we performed an initial clinical experiment to test their functionality. XBP- $1^{f / / f 1} \mathrm{mb} 1 \mathrm{Cre}{ }^{-}$ mice served as control mice in this experiment. As it is suggested that the secretion of pathogenic antibodies is rather mediated by protein antigens than by peptides, we immunized them with 20 $\mathrm{gg}$ rMOG and obtained blood $7 \mathrm{~d}$, 11d and $27 \mathrm{~d}$ p.i. and quantified anti-rrMOG IgM and lgG levels.

A

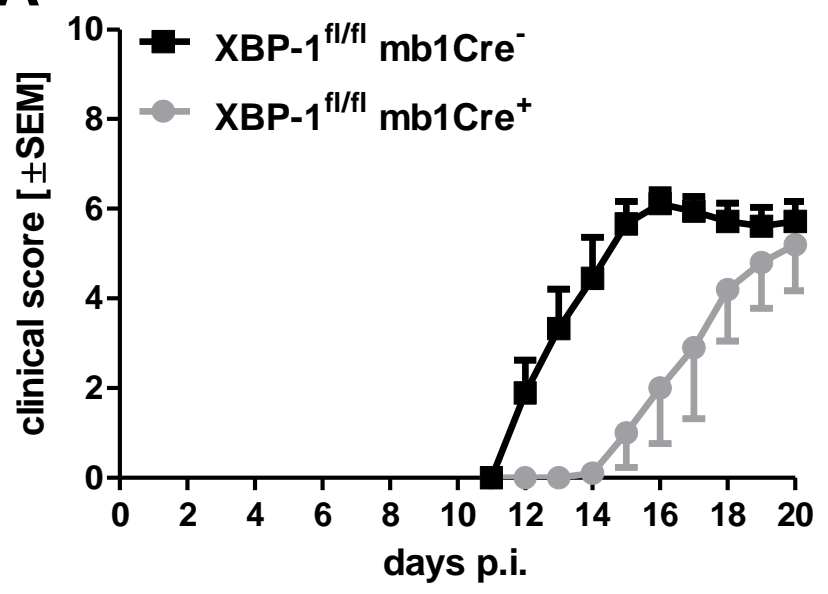

B

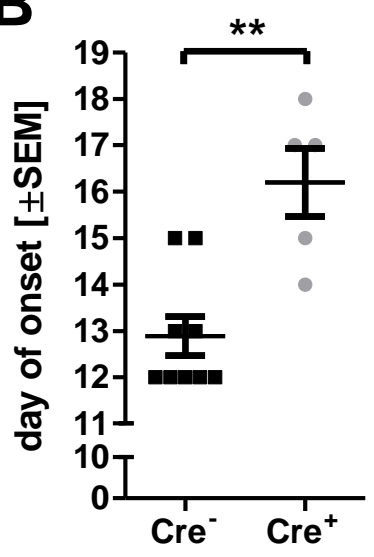

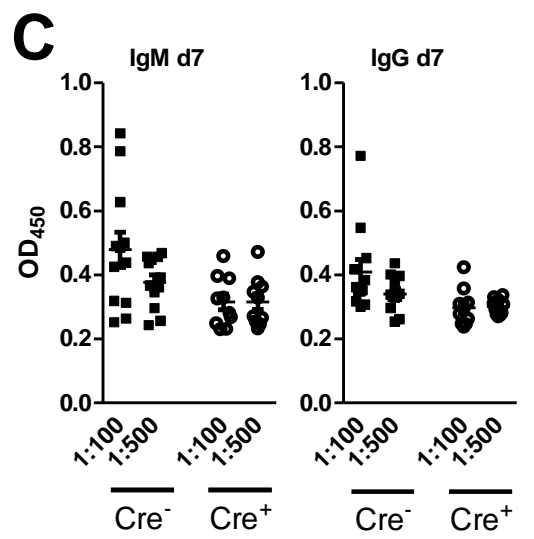
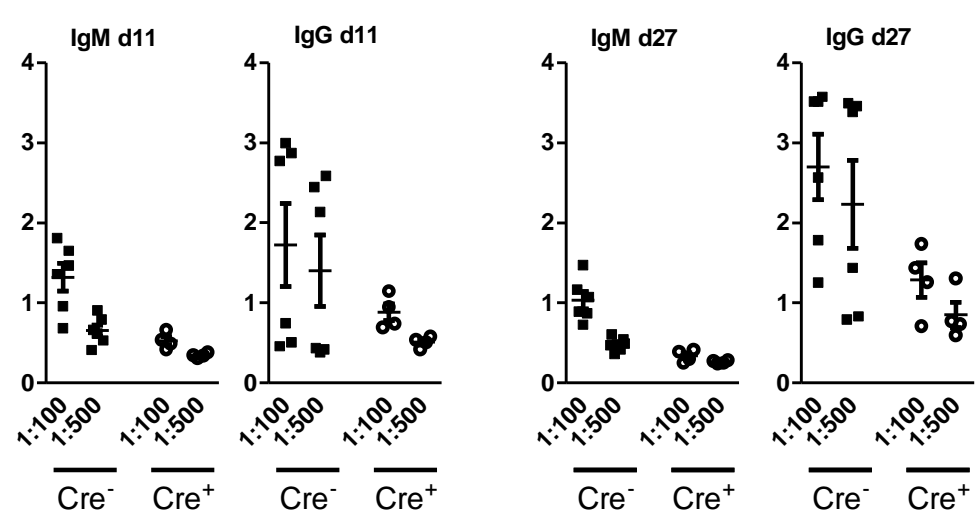

Figure 27: Absence of anti-rrMOG lgs results in a delayed $E A E$ onset. (A+B) Clinical outcome of XBP-1 $1^{\mathrm{fl} / \mathrm{f}} \mathrm{mb} 1 \mathrm{Cre}$ or XBP-1 $1^{\mathrm{fl} / \mathrm{fl}} \mathrm{mb} 1 \mathrm{Cre}^{+}$mice immunized with $20 \mu \mathrm{g}$ rMOG. Healthy mice were not included in the analysis. Clinical data represent 9 animals for Cre group and 5 animals for $\mathrm{Cre}^{+}$group from 2 independent experiments (C) Sera obtained from XBP- $1^{\mathrm{fl} / \mathrm{fr}} \mathrm{mb} 1 \mathrm{Cre}$ or XBP- $1^{f / 1 / 1} \mathrm{mb} 1 \mathrm{Cre}^{+}$mice 7, 11 and $27 \mathrm{~d}$ p.i. and anti-rrMOG IgM and IgG quantification by ELISA.. Each symbol represents an individual mouse in $C$ out of two independent experiments. Data are shown in mean \pm SEM. Significant differences between means are indicated: ${ }^{* *} p<0,01$, Mann-Whitney nonparametric $t$ test. 
During our initial analysis of the XBP-1 $1^{\mathrm{fl} / \mathrm{f}} \mathrm{mb} 1 \mathrm{Cre}^{+}$mice we recognized that these mice had a reduced $B$ cell compartment in contrast to XBP- $1^{\mathrm{fl} / \mathrm{f}} \mathrm{mb} 1 \mathrm{Cre}^{-}$mice. Therefore, the outcome of the clinical data has to be interpreted carefully and might not exclusively be base on the absence of antibodies. But as we planned to use these mice as donor for $B$ cells and not as hosts for cell transfer experiments, the reduced $B$ cell number did not affect our experiments. EAE induction with rrMOG revealed a difference in the clinical outcome of XBP-1-sufficient and XBP-1-deficient $B$ cell mice (Fig. $27 \mathrm{~A}+\mathrm{B}$ ). XBP-1//fll $\mathrm{mb} 1 \mathrm{Cre}-\mathrm{e}^{-}$mice started to develop first clinical signs about four days earlier than XBP-1 ${ }^{1 / 1 / 1} \mathrm{mb} 1 \mathrm{Cre}^{+}$mice (Cre': $12.89 \pm 0.42$; $\mathrm{Cre}^{+}: 16.2 \pm$ $0.73)$. In addition, the different genotype affected the susceptibility for EAE as $47.3 \%$ of $\mathrm{Cre}^{-}$mice developed EAE whereas only $29.4 \%$ of $\mathrm{Cre}^{+}$mice showed clinical symptoms. In line with this was the reduction of anti-rrMOG IgM and IgGs mainly emphasized on day 11 and 27 p.i. (Fig. 27 C). But as mentioned before, we cannot rule out that the observed clinical effect was rather mediated by the reduced $B$ cell compartment than by the lower antibody titer. In addition, it is also questionable whether the reduced antibody titer is due to deficient plasma cell differentiation or the general reduction in $\mathrm{B}$ cell numbers.

\subsubsection{XBP-1-deficient MOG- specific $B$ cells do not accelerate the onset of EAE}

To investigate whether MOG-specific B cells contribute to the accelerated disease onset by their production and secretion of high-affinity anti-rrMOG antibodies of the $\lg G$ isotype, we crossed the XBP-1//fl/ $\mathrm{mb} 1 \mathrm{Cre}^{+}$mice to Th mice (for simplicity designated as "XBP-1 ${ }^{-1-}$ MOG-specific $B$ cells") with the final intention to use these $B$ cells for adoptive transfer experiments. To ensure that the XBP-1 deficiency exclusively affected the humoral immune response of $B$ cells without affecting their cellular immune response, we first tested their antigen-presenting capacity in vitro. We co-cultured them with CFSE-labeled MOG-specific T cells with increasing concentrations of either $M^{M O G}{ }_{p 35-55}$ or rrMOG and analyzed $T$ cell proliferation $72 \mathrm{~h}$ post culture. We additionally assessed MHC-II and CD86 expression of B cells by flow cytometry as readout for $B$ cell activation. 



Figure 28: XBP-1 deficiency does not affect the antigen-presenting capacity of $B$ cells. $1 \times 10^{6}$ MOG-specific T cells were co-cultured with either $2 \times 10^{6}$ MOG-specific $B$ cells (black line) or XBP-1-1- MOG-specific B cells (grey line) with increasing $\mathrm{MOG}_{\mathrm{p} 35-55}$ (left) or rrMOG (right) concentrations $(0 ; 0,01 ; 0,1 ; 1 ; 10 \mu \mathrm{g} / \mathrm{ml})$ for $72 \mathrm{~h}$. (A) T cell proliferation was determined by CFSE dilution. (B+C) Co-cultured B cells were stained for B220 and CD86 (B) or for B220 and MHC-II (C) and analyzed for their mean fluorescence intensity (MFI) of respective marker expression by flow cytometry. Shown is one experiment.

XBP-1 deficiency did not influence the antigen-presenting capacity of MOG specific B cells. Both, XBP-1-deficient and XBP-1-sufficient B cells triggered antigen-specific $T$ cell proliferation at the same antigen concentrations (Fig. 28 A). In addition, both B cell genotypes expressed MHC-II and CD86 with the same kinetic (Fig. $28 \mathrm{~B}+\mathrm{C}$ ). Minor differences in MHC-II expression as observed in Fig. $28 \mathrm{C}$ (right) rather arise from natural variability. Thus, we conclude that the antigen-presenting capacity was not affected by the XBP-1 deficiency and that XBP-1-deficient B cells arise as appropriate $B$ cells to study the role of antibodies in EAE. As the clinical outcome of $T$ and $B$ cell supplementation was more pronounced in the double-transgenic OT-II $x$ NP host mice (Fig. 8 C+D, table 4), we used these mice as hosts for the clinical 
experiment. We transferred MOG-specific T cells either together with MOG-specific B cells or with XBP-1-deficient MOG-specific B cells into OT-II $x$ NP host mice, immunized them two days later with $\mathrm{MOG}_{\mathrm{p} 35-55}$ and monitored their clinical outcome for 20 days.

A



B

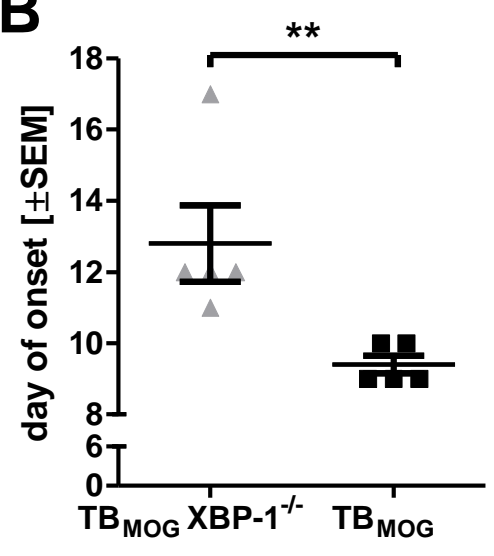

C

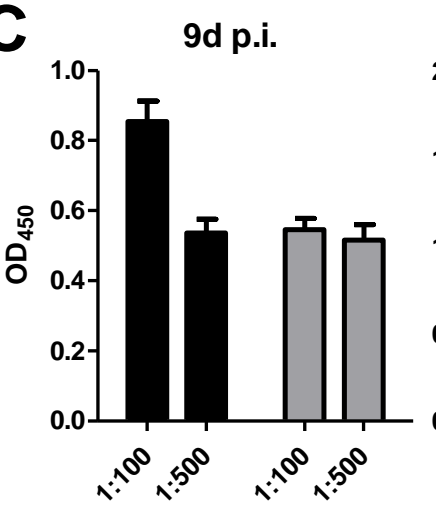

d15-d20 p.i.

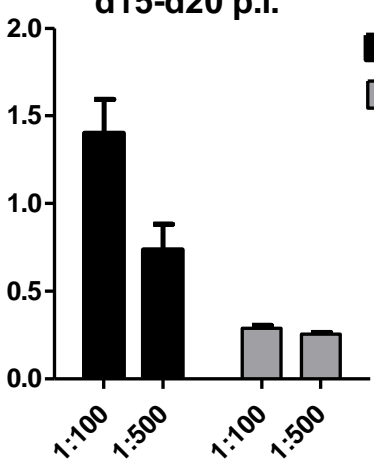

D

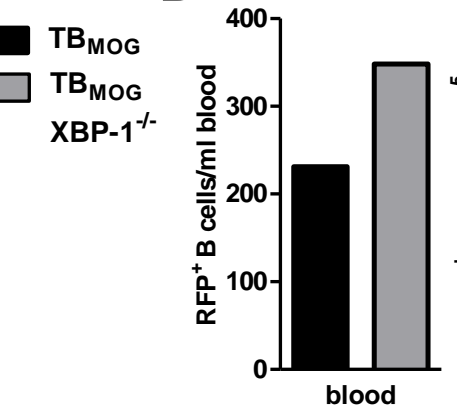

Figure 29: MOG-specific XBP-1-deficient $B$ cells do not accelerate the onset of EAE and do not produce anti-rrMOG specific IgGs. MOG-specific $T$ cells were injected either with RFP ${ }^{+}$MOG-specific B cells or RFP ${ }^{+}$MOG-specific XBP-1-deficient B cells into OT-II $x$ NP mice and immunized with $M_{0} G_{p 35-55}$. (A+B) Clinical outcome of cell transfer (C) Sera obtained from MOGspecific $T$ and $B$ cell-injected mice or MOG-specific $T$ and XBP-1-deficient B cell-injected mice $9 \mathrm{~d}$ p.i. and at end of experiment (depending on the clinical score between d15 and d20 p.i.) and anti-rrMOG $\operatorname{lgG}$ quantification by ELISA (D) Quantification of total RFP ${ }^{+} B$ cell numbers $9 d$ p.i. in blood and spleen. Data in $A$ and $B$ represent 5 mice per group, in $C 2$ mice per group and in $D$ one mouse per group. Data are shown in mean \pm SEM. Significant differences between means are indicated: ${ }^{* *} p<0,01$, Mann-Whitney nonparametric $t$ test.

The transfer of XBP-1/ MOG-specific $B$ cells did not accelerate the disease onset like observed with XBP-1-sufficient MOG-specific B cells (Fig. 29 A+B). Precisely, mice which received MOG-specific $T$ cells and XBP-1-deficient $B$ cells developed first clinical signs at about day $12.8 \pm 1.0$, whereas the transfer of MOG-specific $T$ cells together with XBP-1- sufficient B cells resulted in a clinical onset at about day 9.4 \pm 
0.24. In contrast to that, the different $B$ cell genotype did not impact the susceptibility to $E A E$, as both groups developed EAE with an incidence of $83 \%$. To ensure that XBP-1-deficient $B$ cells were really impaired in antibody production, we obtained blood from two animals per group 9 days p.i. and from all animals after they had to be sacrified due to a too high clinical score and determined serum anti-rrMOG IgG level. Mice which received XBP-1-sufficient MOG-specific B cells showed an increased anti-rrMOG IgG serum level already 9 days p.i. (Fig. $29 \mathrm{C}$, left), which was even enhanced at later time points, whereas serum anti-rrMOG IgG levels of XBP-1deficient $B$ cell-injected mice did not exceed background level (Fig. $29 \mathrm{C}$, right). As mentioned before, Th XBP- $1^{f / 1 / 1}$ mb1Cre ${ }^{+}$mice (donor mice of MOG-specific XBP-1deficient $B$ cells) have an impaired $B$ cell compartment with low numbers of peripheral $B$ cells. To exclude that the observed clinical effect and the accompanied low serum anti-rrMOG IgG level was due to an e.g. increased apoptosis rate of the transferred $B$ cells, we determined the number of transferred $\mathrm{RFP}^{+} \mathrm{B}$ cells of one mouse per group 9 days p.i.. At that time point, the number of transferred $B$ cells was similar in the spleen and in the blood (Fig. $29 \mathrm{D}$ ) between the two groups, leading to the conclusion that the deficiency of XBP-1 rather affected the development of $B$ cells, but did not impact the survival of mature $B$ cells with the consequence that isolated $B$ cells stay viable upon transfer. In summary with the in vitro data, these data provide evidence that the transferred MOG-specific B cells promote the development of EAE through the secretion of MOG-specific IgGs. However, how these antibodies exert their pathogenic potential remains elusive. 


\section{Discussion}

\subsection{Establishment of the EAE model}

Although MS is known to be a $T$ cell-mediated autoimmune disease, beneficial effects of B cell-depleting therapies of MS patients with Rituximab, an anti-CD20 monoclonal antibody, reveal evidence that $B$ cells are involved in the pathogenesis of MS. Several publications disclose divergent roles for B cells during the course of MS and EAE, the mouse model of MS. On the one hand, B cells can exert their proinflammatory function by their secretion of pro-inflammatory cytokines $[55,56,154]$ or by triggering $T$ cell activation through antigen-presentation [139]. Moreover, autoantibodies are also considered to be involved in $\operatorname{MS}$ and $\operatorname{EAE}[53,54,140]$. On the other hand, a regulatory subpopulation of $B$ cells and plasma cells were shown to secrete anti-inflammatory cytokines like IL-10 and IL-35 and thus dampen down the autoimmune response $[157,159,160]$. In addition to the different roles suggested for $B$ cells, the critical time point when $B$ cells participate in the immune response also seems to be uncertain. Whereas some publications suggest a role for $B$ cells in $T$ cell priming in secondary lymphoid tissues [139], others suggest a critical role for B cells in the reactivation phase of EAE in the CNS [154]. Similarly, the critical time point of regulatory $B$ cell contribution also seems to be controversial. Whereas some reports reveal a critical requirement for regulatory $B$ cells during the EAE recovery phase [157], others suggest an important role for these cells in the EAE induction phase [161]. Hence, there is an increasing interest in studying the role and the critical time point of B cell contribution to the pathogenesis of MS. This would help to develop drugs exclusively targeting the $\mathrm{B}$ cell-dependent mechanism without affecting the total cell lineage.

We have now developed a system enabling us to track and analyze $T$ and $B$ cells of the same antigen-specificity in vivo during the course of EAE. Fluorescently labeled (RFP or GFP) MOG-specific T and B cells were isolated from respective donor mice and injected i.v. into different recipients followed by immunization with MOG peptide $\left(M G_{p 35-55}\right)$. This allowed the re-isolation and characterization of the cells during the whole course of actively induced EAE from the periphery, as well as from the CNS. We did not manipulate the course of EAE by transferring e.g. activated T cells (Fig. 4) and the antigen-specific $T$ cells responded specifically to their appropriate antigen in vivo (Fig. 5). This ensured that the injected cells became primed in vivo in a similar 
manner as the endogenous lymphocytes and that co-injected $T$ and $B$ cells could interact with each other. We first established the model in C57bl/6 WT mice, where we observed a disease promoting effect of transferred MOG-specific T cells, but also of transferred MOG-specific B cells (Fig. $7 \mathrm{~A}, \mathrm{C}$ ), leading to the assumption that both lymphocyte populations participate in the autoimmune response. Thus, we thought that the simultaneous transfer of MOG-specific $T$ and $B$ cells might even enhance the observed effects. Although we observed a trend that the simultaneous transfer of MOG-specific $T$ and $B$ cells accelerate and exacerbate the course of disease in comparison to MOG-specific $T$ cell transfer alone, the difference was not statistically significant (Fig. 7 A, B). We assumed that the contribution of the endogenous MOGspecific $T$ cell pool to the disease progression was too strong to further significantly enhance the immune response. Therefore, we employed OT-II host mice, as it is known that OVA-specific $T$ cells do not respond to MOG antigens and our in vitro data confirmed this observation (Fig 6 A left and middle). In line with this, OT-II mice were not susceptible for EAE induction with $M_{0 G_{35-55}}$ (Fig. $8 \mathrm{~A}$ ), leading to the conclusion that the participation of OVA-specific $T$ cells in the autoimmune response against $M O G$ and thus in the initiation of $E A E$ is negligible. To minimize the endogenous $T$ and $B$ cell effects simultaneously, we crossed the OT-II mice to NP mice resulting in OT-II $\times$ NP mice. These mice, mainly harboring OVA-specific T cells and NP-specific $B$ cells, enabled us to widely exclude any effects from endogenous $T$ and $B$ cells after immunization with MOG peptide. As observed with OT-II mice, OT-II $x$ NP mice were not susceptible for EAE induction either. The protection of EAE could be overcome by the transfer of MOG-specific T cells (Fig. $8 \mathrm{~A}$ ) in OT-II host mice, as well as in OT-II x NP host mice (Fig. $8 \mathrm{C}$ ). In both models, the additional transfer of MOG-specific $B$ cells to MOG-specific $T$ cells significantly accelerated the disease onset and increased the susceptibility for EAE (Fig. 8 A-D, Table 4), although the disease-accelerated effect of MOG-specific B cell supplementation was more pronounced in the OT-II $\times$ NP model (Table 4, day of onset). Thus, both models exhibited several advantages: First, they facilitated a focus on the pathogenic MOGspecific $T$ and $B$ cell population during the course of EAE, not only in the periphery, but also in their target organ, the CNS. Second, they permitted us to study the impact of MOG-specific $B$ cells on the course of EAE, because the accelerated onset revealed an effect of the transferred MOG-specific B cells in OT-II mice and, even more pronounced, in OT-II $\times$ NP mice. Furthermore, we avoided the usage of host 
mice lacking e.g. $B$ cells $(\mu M T)$ or even $T$ and $B$ cells $\left(\right.$ RAG2 $\left.^{-1}\right)$ as these mice are known to have an impaired secondary lymphoid organ structure and injected $T$ cells can undergo homeostatic proliferation $[185,186]$. The traditional view is that peptide antigens are predominantly presented by professional APCs like DCs, whereas B cells require the presence of protein antigens for presentation [187, 188]. In line with this, several publications reveal that the presence or absence of $B$ cells in MOGpeptide induced $E A E$ is completely irrelevant for the course of $E A E[138,139]$. Our own observations showed the reverse. We could clearly show that the supplementation of C57bl/6 WT mice with MOG-specific B cells (Fig. 7 C) or the simultaneous transfer of MOG-specific T and B cells into OT-II and OT-II X NP mice and subsequent MOG peptide immunization accelerated the onset of EAE and increased susceptibility in comparison to non-supplemented mice or $\mathrm{T}$ cellsupplemented mice, respectively (Fig. 8 A-D). We also established the above described models (C57bl/6 WT and OT-II) with rMOG protein immunization (data not shown) and also observed an effect of supplemented MOG-specific B cells on the disease course. However, we were more interested in the role of $B$ cells in MOG peptide induced EAE, because this is contradictory to most of the publications [138, 139]. It is suggested in the literature that peptide antigens can be presented by $B$ cells irrespective of their antigen receptor specificity by binding on surface MHC-II molecules $[78,79,139]$. Our in vitro results also reveal that NP-specific B cells can trigger MOG-specific $T$ cell proliferation in the presence of $M_{0} G_{p 35-55}$ almost as efficiently as MOG-specific B cells (Fig. $6 \mathrm{~A}$ left), whereas they fail to induce $T$ cell proliferation in the presence of rMOG (Fig. 6 A middle). This led to the conclusion that the antigen-specificity of the $B$ cells is irrelevant for triggering $T$ cell proliferation in the presence of MOG peptide antigens in vitro. However, the simultaneous transfer of MOG-specific T cells and NP-specific B cells into OT-II hosts and subsequent immunization with $\mathrm{MOG}_{\mathrm{p} 35-55}$ did not lead to the accelerated disease onset observed with MOG-specific B cells (preliminary data, Suppl. Fig. S1). This led to the assumption that the in vivo interaction of MOG-specific $T$ and $B$ cells in the presence of $\mathrm{MOG}_{\mathrm{p} 35-55}$ did not simply affect $\mathrm{T}$ cell proliferation, but rather might result in a more complex impact on the entire autoimmune response. In line with this, we observed a diminished EAE susceptibility of T cell-injected OT-II x NP mice in contrast to T cellinjected OT-II mice (Table $4 ; 61.5 \%$ vs. 35.7\%). This observation also emphasizes the critical role of $B$ cell receptor antigen-specificity because if the antigen-specificity 
of the endogenous $B$ cell pool is irrelevant for peptide-induced $E A E$, it might not affect the clinical outcome of EAE. It should be mentioned here that we did not experimentally exclude a difference in the residual antigen-presenting cell pool of the two different host mice, which could possibly also account for a different EAE susceptibility.

In summary the newly established EAE model not only enabled us to study the behavior of MOG-specific $T$ and $B$ cells during the course of EAE, but also highlights the importance of antigen-receptor specificity even in MOG peptide-induced EAE.

\subsection{The role of MOG-specific $B$ cells in the activation phase of EAE}

There are several possible mechanisms of how $B$ cells can influence the course of $E A E$. It is considered that $B$ cells act as antigen-presenting cells and thereby prime encephalitogenic $T$ cells [139]. In addition, B cells influence $T$ cell differentiation by their provision of pro-inflammatory cytokines such as IL-6 [56]. Moreover, B cells can secrete high affinity anti-MOG-specific antibodies [140]. In contrast to the described pro-inflammatory roles for $B$ cells in EAE, several publications also suggest a regulatory role for some $B$ cell subpopulations during disease progression $[159,160]$. However, in all of our experimental models (C57bl/6 (Fig. 7), OT-II, OT-II x NP hosts (Fig. 8)), we observed a disease promoting effect of the transferred $B$ cells and in none of them did we find an implication for a regulatory role of $B$ cells. Furthermore, we tried to identify regulatory $B$ cells $\left(C D 19^{+} C D 1 d^{\text {hi }} C D 5^{+}\right)$by FACS staining in naïve C57bl/6 and Th mice and could not at all detect a distinct population. Therefore, we rather suggest a pro-inflammatory role for the transferred $B$ cells in our system. We were interested whether the transferred MOG-specific $B$ cells participate as antigenpresenting cells in the priming phase (d2- d4) of EAE. We rather expected a supportive than a critical role of the $B$ cells, as the role of $B$ cells in priming naïve $T$ cells in vivo is controversially discussed $[189,190]$ and conditional MHC-II expression of $B$ cells in EAE also reveals a limited role for $B$ cells as antigen-presenting cells [38]. In line with this we observed that the transfer of MOG-specific $T$ cells alone is sufficient to drive the development of EAE. When we focused on $T$ cell proliferation between $\mathrm{d} 2$ and $\mathrm{d} 4$, we could not detect a difference of $\mathrm{T}$ cells injected alone or together with MOG-specific B cells (Fig. $10 \mathrm{~A}+\mathrm{B}$ ), which was in line with the $T$ cell 
number (Fig. $10 \mathrm{C}$ ) and frequency among the $\mathrm{CD} 4^{+} \mathrm{T}$ cell pool (Fig. $10 \mathrm{D}$ ). Moreover, the presence of MOG-specific $B$ cells did not increase the frequency of activated $T$ cells (Fig. 11). In general, antigen-presentation by $B$ cells can induce $T$ cell differentiation into a T helper cell direction [191] and several publications suggest that B cells promote predominantly TH17, but also TH1 differentiation in EAE [56, 139, 151]. However, we did not detect a difference in IFN $\gamma$, IL-17A or GM-CSF expression on mRNA level early after disease induction (2.5d p.i.; Fig. $12 \mathrm{~A}$ ) and later (4d p.i.; Fig. 12 B). Furthermore, the presence of $B$ cells neither expanded already differentiated $T$ cells nor increased their sensitivity to lower antigen concentrations (Fig. 13). Analyses with MOG-specific FOXP3-GFP reporter T cells also revealed no impaired conversion of naïve $T$ cells into induced regulatory $T$ cells in the presence of B cells (Suppl. Fig. S2).

From these observations we conclude that MOG-specific $B$ cells did not modulate $T$ cell proliferation, activation or differentiation during the priming phase of $E A E$.

\subsection{The role of MOG-specific B cells before onset of EAE}

As we could not detect a contribution of MOG-specific B cells to promoting encephalitogenic $T$ cell amplification, activation or differentiation in the priming phase of $E A E$, we focused on the time point immediately before $E A E$ onset. We know from experiments with inducible conditional CD28 knockout mice that $T$ cell priming is not entirely completed after the activation phase of EAE [212]. Thus, it is possible that $B$ cells exert their pro-inflammatory function later during preclinical progression. $A s B$ cells accelerate EAE onset from day 11 to day 9 in our EAE model (Fig. 8 A+B, Table 4), we hypothesized that we would find a difference in the T cell phenotype shortly before the manifestation of clinical symptoms of MOG-specific $T$ and $B$ cell-injected mice. We used the technique of two-photon intravital imaging to distinguish mice with intraluminal and extraluminal T cells in the CNS. Almost all of the analyzed $T$ and $B$ cell-injected mice were found to have already extravasated $T$ cells, which resembled the clinical outcome (Fig. 15 A). However, when we compared T and B cell-injected mice with extravasated $T$ cells and $T$ cell-injected mice without extravasated $T$ cells with further flow cytometric analyses we did not detect any difference in the peripheral T cell number (Fig. 15 B) or frequency of activated T cells (Fig. 15 C) 
between the two groups. This was in line with a study published where no difference could be detected in peripheral T cell activation in the absence or presence of MOGspecific $B$ cells of healthy mice [78]. As mentioned previously, it is traditionally thought that $B$ cells drive the development of TH17 cells $[56,139]$. We could not detect any difference in the expression level of IFN $\gamma$, IL-17A and GM-CSF on mRNA level of peripheral MOG-specific T cells 9d p.i. (Fig. 16 A). In addition, there was no difference in cytokine secretion of restimulated lymph node cells and splenocytes reisolated either from $T$ cell or $T$ and $B$ cell-injected mice before clinical manifestation of EAE (9d p.i.) (Fig. 16 B-D). However, we found increased levels of IFN $\gamma$ and IL-17A (Fig. $16 \mathrm{~B}, \mathrm{C}$ ) from sick $T$ and $B$ cell-injected mice (also re-isolated $9 \mathrm{~d}$ p.i.). Notably, most of the publications which suggest a role for $B$ cells in driving $T$ cell differentiation, analyzed cytokine expression in late phases of disease progression (between d14 and 32 p.i.) when mice already display a difference in the clinical progress $[56,139,151]$. But whether this was a specific B cell effect or due to the overall increased pro-inflammatory milieu remains questionable.

When we focused on the underlying reason why $T$ and $B$ cell-injected mice displayed an accelerated EAE onset, we could clearly detect an increased number of CNSinfiltrated encephalitogenic $T$ cells (Fig 17). As we could never detect any of the injected MOG-specific B cells in the CNS of healthy mice (by flow cytometry or by two-photon imaging performed by my colleague Tanja Litke) and just very few of them in mice with fulminant EAE (Score 4.5) (Fig. 18 B), we exclude a role at least for the injected $B$ cells in the reactivation step. This was in contrast to a recent publication, which suggests that the initial interaction of $T$ and $B$ cells in the CNS account for an enhanced pro-inflammatory milieu leading to further cell attraction [154]. Moreover, the presence of meningeal ectopic $B$ cell follicles was also reported in some MS patients [51]. But in our study also initial CNS-infiltrated T cells $(\sim 3000$ cells) isolated before the occurrence of clinical signs, displayed similar IFN $\gamma$, IL-17A and GM-CSF mRNA levels (Fig. $18 \mathrm{~A}$ ). This was a quite challenging analysis, because it was not feasible to accurately identify the first 50-100 infiltrated cells. Therefore we cannot completely exclude that the very first infiltrated $T$ cells became reactivated more efficient in $T$ and $B$ cell-injected mice, which would then lead to further cell attraction with the consequence of a higher number of detected infiltrated cells before the onset of EAE. Our analyses did clearly show that the earlier onset was due to a higher number of infiltrated $T$ cells and not due to a general enhanced 
reactivation in the presence of $B$ cells. However, the higher number of infiltrated cells was neither accompanied by an increased peripheral $\mathrm{T}$ cell number, nor by an enhanced T cell activation or differentiation in the periphery.

\subsection{Effect of MOG-specific B cells on $T$ cell migration}

The migration behavior of $\mathrm{T}$ cells is determined by their expression of distinct chemokine receptors and adhesion molecules. It is widely accepted that expression of CCR9 and LPAM (integrin $\alpha_{4} \beta_{7}$ ) by T cells is responsible for their gut homing [192194], whereas skin-homing is mediated by $E$ and P-selectin ligands and CCR4 and CCR10 [195, 196]. The upregulation of the particular homing receptors is mediated by the interaction of $T$ cells with respective tissue-specific dendritic cell subsets [180, 197]. Chemokine receptor-dependent T cell attraction is also critically involved in the pathogenesis of EAE. Whereas TH1 cells preferentially utilize CXCR3 and CCR5, TH17 cells are characterized by their expression of CCR6. Interference or complete absence of the chemokine receptors or their ligands results in attenuated disease course or complete resistance to $\operatorname{EAE}[120,121,135]$. Beside the upregulation of pro-inflammatory chemokine receptors, also homeostatic chemokine receptors such as CCR7 and CXCR4 have been shown to be involved in CNS migration [103, 120, 130]. Therefore, the critical chemokine receptor required for CNS infiltration does not seem to be as strictly defined as observed in gut and skin homing. To get an idea which chemokine receptor was preferentially utilized by the pathogenic $T$ cells in our model, we determined chemokine receptor expression of initial infiltrated $T$ cells on mRNA level. We did not detect a statistically significant difference in chemokine receptor expression of $T$ cells either injected alone or simultaneously with MOGspecific B cells. However, there was a tendency of a higher CCR7 and CCR6 expression (Fig. 19) of T cells injected together with B cells. Interestingly, these cells also showed an enhanced, but not statistically different, expression of the $\mathrm{TH} 17$ specific cytokines IL-17A and GM-CSF (Fig. 18 A). Therefore, one can argue that the peripheral interaction of MOG-specific $T$ cells with MOG-specific $B$ cells somehow drive the development of the TH17 phenotype. It is possible that the experimental approaches we used (RT-PCR of sorted T cells from periphery and in vitro antigenspecific restimulation) to determine $T$ helper cell differentiation in the periphery before clinical onset were not sensitive enough to detect already differentiated $\mathrm{TH} 17$ cells or 
that these cells were not terminally differentiated into $\mathrm{TH} 17$ cells before they became reactivated in the CNS by perivascular APCs. In agreement with this finding, we also detected an increase in CCR6 expression of splenic T cells (Fig. 20). CCR6 ${ }^{+}$CD4 T cells (TH17 cells) have been shown to be crucial for the initiation of EAE. They infiltrate the CNS at the site of the choroid plexus into the brain, where their reactivation results in an overall increased pro-inflammatory milieu whereby they trigger further CCR6-independent lymphocyte infiltration [135]. As we also detected increased T cell numbers in the brain before the onset of clinical EAE (Fig. $15 \mathrm{~B}$ ), this scenario can also account for the accelerated onset in our experimental model. We are planning to investigate this observation in detail, for example by determining the number of T cells in the CSF. Furthermore, we observed a significant upregulation of CXCR4 of $T$ cells re-isolated from peripheral lymphoid organs and blood (Fig. 20). The role of CXCR4 and its ligand CXCL12 in the context of EAE is controversial. CXCL12 is expressed by spinal cord endothelial cells, among other sites, where it is mainly secreted at the basolateral surface into the perivascular space. Here, it has been suggested, CXCL12 traps CXCR4 $4^{+}$lymphocytes and prevents their migration deeper into the CNS parenchyma [130]. Although treatment studies with AMD3100, an antagonist of CXCR4 signaling, reveal worsening of clinical symptoms due to an increased number of infiltrated mononuclear cells deeper into parenchymal tissue after treatment, CXCR4 blockade had no effect on the onset of the disease. Therefore, it is also likely that a different CXCR4 expression kinetic of the pathogenic $T$ cells can account for the earlier disease onset observed in our experimental model. In theory, the CXCR4 ${ }^{+} \mathrm{T}$ cells become attracted into the perivascular space, where they become reactivated by resident APCs, which in turn leads to the upregulation of various chemokines and cytokines enabling further cell infiltration. In line with this, we observed massive upregulation of CXCL10 and CCL5 on mRNA level of spinal cord microvessel endothelial cells isolated 9-11d p.i. when initial CNS infiltrates were detected in T and B cell-injected, but also in some T cell-injected mice (Suppl. Fig S3). The absent CXCR4 expression of the initial infiltrated cells (Fig. 19) can be explained by a downregulation during reactivation. However, it is experimentally challenging to find further evidence supporting our CXCR4 theory, as functional interference with CXCR4 would mainly target the altered T cell trapping rather than the initial infiltration. It is feasible to re-isolate $\mathrm{CXCR} 4^{+} \mathrm{RFP}^{+} \mathrm{T}$ cells shortly before EAE onset from the periphery, transfer them into new host mice and investigate 
whether they infiltrate the CNS earlier than control T cells, but it is questionable whether they maintain their CXCR4 expression until they arrive at the BBB.

As integrin expression by T cells is also critically required for CNS infiltration, we also focused on integrin $\alpha_{L}$ (LFA-1) and integrin $\alpha_{4}$ (VLA-4) expressions before onset of EAE (Fig. $21 \mathrm{~A}$ ). We detected neither an enhanced expression on mRNA level nor an increased number of VLA-4 ${ }^{+} \mathrm{T}$ cells (Fig. $21 \mathrm{~B}$ ) or LFA-1 ${ }^{+} \mathrm{T}$ cells (Fig. $21 \mathrm{C}$ ). However, this was not surprising, as LFA-1 and VLA-4 are not exclusively involved in $T$ cell migration, but are also involved in T cell-APC interaction, leading to several further factors which might influence their expression. Moreover, it is also likely that $B$ cells do not influence the integrin expression of $T$ cells, but rather their activation status. LFA-1 is normally expressed in a quiescent state on the surface of leukocytes and interacts weakly with its ligands ICAM-1 [198, 199]. Upon activation, e.g. by chemokine receptor signaling, it changes its conformational state into an active form, leading to a firm interaction with its endothelial cell expressed ligand [200]. As LFA-1 is also involved in the forming of an immunological synapse between $T$ cells and APCs, it is in general possible that B cells can influence its activation state. At the moment we are establishing an in vitro BBB approach to test whether antigenspecific $T$ and $B$ cell interaction results in a higher number of firmly adhesive $T$ cells.

\subsection{Effect of MOG-specific B cells on myeloid cells}

It is widely accepted that other leukocyte subsets are also critically involved in the pathogenesis of EAE. Myeloid cells do not only mediate tissue damage through secretion of toxic factors, but they also serve as antigen-presenting cells for the reactivation of infiltrated myelin-specific T cells [201-204]. Therefore, an absence of myeloid cell infiltration due to impaired chemokine signaling leads to decreased $T$ cell infiltration and overall reduction in produced pro-inflammatory mediators [165-168]. The CD11 b ${ }^{+}$Ly6 $C^{\text {hi }}$ monocyte population is critically involved in EAE pathogenesis. This population is released from the bone marrow into the blood immediately before EAE relapse in a GM-CSF-dependent manner. Upon CNS infiltration the cells upregulate MHC-II and gain pro-inflammatory functions. Furthermore, the increase in circulating Ly6C ${ }^{\text {hi }}$ monocyte number was accompanied by an accelerated disease onset and an increase in clinical severity [169, 181]. As we did not detect a difference 
in GM-CSF expression of the injected MOG-specific $T$ cells after activation phase and before EAE onset (Fig. 12B, 13C, 16 A/D), we exclude that mechanism as accounting for a possible increase in monocyte number. However, it was shown that $B$ cells attract monocytes to the heart after myocardial infarction in a CCL7dependent manner. Therefore, we also wondered whether B cells can also influence monocyte mobilization in an autoimmune disease. We detected an increase in Ly6 $\mathrm{C}^{\mathrm{hi}}$ monocyte number in the blood of $\mathrm{T}$ and $\mathrm{B}$ cell-injected mice $7 \mathrm{~d}$ p.i., but not in spleen and spinal cord (Fig. 22 B). However, this increase was not consistent during the following days. One can argue that the number of Ly $6 \mathrm{C}^{\text {hi }}$ monocytes diminished in the blood of $T$ and $B$ cell-injected mice due to their enhanced infiltration into the CNS (Fig. $22 \mathrm{C}+\mathrm{D}$ ), but one would assume a subsequent increased egress of monocytes from the bone marrow into the blood to compensate the reduced monocyte number in the blood. In summary, in our experiments the monocyte numbers were not as strikingly different as described in the literature. Therefore, we found no evidence that this mechanism is responsible for the accelerated disease onset.

\subsection{Effect of MOG-specific B cells on spinal cord microvessel endothelial cells}

CNS T cell infiltration can not only be influenced by their chemokine receptor expression, but also by chemokine and adhesion molecule expression of endothelial cells from spinal cord microvessels. The expression of chemokines and adhesion molecules is mainly regulated by different cytokines, for example IFN $\gamma$ or IL-6 [183, 205-207]. As several chemokines become upregulated before the appearance of first clinical signs, it is possible that their different expression kinetic can account for an accelerated disease onset [183, 205-207]. However, we did not detect a difference in the expression of adhesion molecules (ICAM-1, VCAM-1, ALCAM) by spinal cord microvessel endothelial cells isolated from $T$ cell or $T$ and $B$ cell-injected mice before first $\mathrm{T}$ cell infiltration (8d p.i.) (Fig. $23 \mathrm{~A}$ ). In addition, sorted endothelial cells also displayed similar chemokine expression levels (Fig. $23 \mathrm{~B}$ ), leading to the conclusion that the presence of MOG-specific B cells did not trigger $T$ cell infiltration by influencing the expression of adhesion molecules and chemokines by spinal cord microvessel endothelial cells. However, we could confirm the observation that some 
chemokines became upregulated before the appearance of clinical signs. When we determined adhesion molecule and chemokine expression of healthy animals with infiltrated cells, we observed an enhanced chemokine expression level, specifically of CXCL10 and CCL5, but this was a secondary effect due to the ongoing reactivation of infiltrated T cells (Suppl. Fig S3).

\subsection{The antigen-specific interaction of $T$ and $B$ cells determine the accelerated disease onset}

B cells can also be involved in the establishment of a pro-inflammatory milieu, not only by their antibody secretion or antigen-presenting capacity which would require antigen-specificity, but also by their secretion of cytokines, which are crucial to drive $T$ cell differentiation $[55,56,139]$. Hence, also activated autoantigen-unspecific $B$ cells can be involved in the initiation of an immune response [208, 209]. However, in the context of EAE, it was reported that the transfer of activated MOG-unspecific $B$ cells cannot restore the EAE susceptibility of $\mu \mathrm{MT}$ mice as observed with transfer of activated MOG-primed B cells [140]. Therefore, we were also interested whether or not the presence of activated but antigen-unspecific $B$ cells is sufficient to accelerate the onset of $E A E$ in our experimental setting. The simultaneous immunization with NP-OVAL and $M \mathrm{GG}_{p 35-55}$ ensures the activation of the transferred NP-specific B cells in presence of the endogenous OVA-specific $T$ cells while also ensuring a sufficient priming of the transferred MOG-specific $T$ cells. We confirmed the successful $B$ cell activation by detection of a high serum anti-NP $\lg G$ titer (Fig $24 \mathrm{C}$ ). However, activated MOG-unspecific $B$ cells failed to accelerate the EAE onset compared to MOG-specific $B$ cells (Fig. 24 A+B). This finding again emphasized our observation that MOG-specific B cells play a supportive role even in MOG peptide-induced EAE. One can argue that the antigen-specific interaction of OVA-specific T cells and NPspecific $B$ cells results in a different cytokine production profile than the antigenspecific interaction of MOG-specific T and B cells. Thus, we determined the cytokine levels of in vitro co-cultured $\mathrm{T}$ and $\mathrm{B}$ cells and even detected elevated levels of proinflammatory cytokines such as IFN $\gamma$, TNF $\alpha$ and IL-6 in OVA-specific T cell and NPspecific B cell co-culture (Suppl. Fig S4). 


\subsection{The role of antibodies in $M O G_{p 35-55}$ induced EAE}

Previous experiments reveal that activated, MOG-unspecific $B$ cells were not able to accelerate the onset of EAE as observed with MOG-specific B cells. Thus, we wondered about the disease accelerating mechanism which might be based on the antigen-specific interaction of $T$ and $B$ cells. Obviously, the MOG-specific interaction results in a different humoral immune response than the NP-OVAL-specific interaction. Although several publications suggest a dispensable role for $\mathrm{B}$ cells and auto-antibodies in MOG peptide-induced EAE [139, 140, 150, 151], we detected antirrMOG, but not anti-MOG $\mathrm{p}_{35-55}$ antibodies of the $\mathrm{IgG}$ isotype after MOG peptide immunization secreted by the transferred MOG-specific $B$ cells before appearance of clinical signs (Fig 26. A). The anti-rrMOG IgG secretion required the presence of MOG-specific $T$ and $B$ cells suggesting that these cells interacted in vivo (Fig. $26 \mathrm{D}$ ). Our observation was supported by a publication which showed that B cell proliferation and isotype class switching can be induced by MOG-specific TH17 cells in vitro in the presence of $M_{0 O} G_{p 5-55}$ [210]. The fact that we detected anti-MOG protein-specific IgGs even though we immunized with MOG peptide was presumably due to the specificity of the knock-in antibody of the transferred MOG-specific B cells. These antibodies were shown to accelerate and exacerbate EAE [143]. The role of antibodies in C57bl/6 MOG-induced EAE is controversial. Whereas a recent publication suggests a dispensability for antibodies upon rhMOG-immunization as well as for the development of spontaneous EAE [139], another study reveals a critical requirement for rhMOG-primed serum in restoring the susceptibility of EAE in $\mu M T$ mice [140]. In addition, the presence of high endogenous MOG-specific serum autoantibody titer was shown to enhance clinical effects [143]. To investigate whether MOG-specific IgGs accounted for the accelerated disease onset in our experimental system, we employed XBP- $1^{f / f \mid l} \mathrm{mb}-1 \mathrm{Cre}{ }^{+} \mathrm{MOG}$-specific $\mathrm{B}$ cells for adoptive transfer experiments. XBP-1 is a transcription factor required for the terminal differentiation of $\mathrm{B}$ cells to plasma cells. In the literature it is described that XBP-1/ $\mathrm{B}$ cell mice displayed low baseline serum levels of all $\lg$ isotypes and an impaired humoral immune response upon immunization, but XBP-1-deficiency did not affect B cell activation and proliferation in vitro as well in vivo [184]. When we immunized XBP-

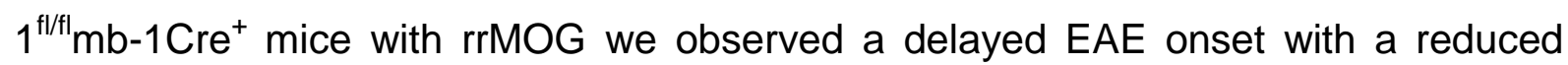
disease severity and susceptibility compared to XBP-1 ${ }^{f / f t} \mathrm{mb}-1 \mathrm{Cre}$ control mice (Fig. $27 \mathrm{~A}+\mathrm{B})$, which was accompanied by reduced serum anti-rrMOG IgM and IgG levels 
at several time points during disease progression (Fig. $27 \mathrm{C}$ ). Although peripheral B cell numbers were not affected when the Cre recombinase was expressed under the B cell-specific CD19 promotor [211], we detected reduced numbers of peripheral B cells in XBP-1 ${ }^{\mathrm{fllfl}} \mathrm{mb}-1 \mathrm{Cre}{ }^{+}$mice (as well as in Th XBP-1 ${ }^{\mathrm{fllfl}} \mathrm{mb}-1 \mathrm{Cre}{ }^{+}$mice). Therefore, it is not clear whether the clinical differences arose from the reduced $B$ cell number or from the reduced antibody levels. When we adoptively transferred MOG-specific $\mathrm{XBP}-1^{\mathrm{f} / \mathrm{fl}} \mathrm{mb}-1 \mathrm{Cre}^{+} \mathrm{B}$ cells, we observed that they did not accelerate the disease onset after $\mathrm{MOG}_{\mathrm{p} 35-55}$ immunization like observed with XBP-1-sufficient MOG-specific $B$ cells (Fig. $29 A+B$ ). The delayed disease onset was accompanied by reduced antirrMOG IgG serum levels before establishment of clinical EAE as well as on later time points (Fig. 29 C) without affecting transferred peripheral B cell numbers (Fig. 29 D). Our in vitro data reveal that XBP-1-deficiency did not influence the antigen-presenting capacity or activation of $B$ cells (Fig. $28 \mathrm{~A}-\mathrm{C}$ ), assuming that the diminished humoral immune response accounted for the delayed clinical onset. However, one should keep in mind that the XBP-1 deficiency impairs the development of plasma cells and does not affect the antibody secretion directly. Since a recent publication reveals a novel additional role for $\mathrm{CD}_{138^{+}}$plasma cells during EAE and infectious diseases, it is also possible that plasma cells contribute to the accelerated onset in a different way than by the secretion of antibodies [159]. It remains elusive how these antibodies exert their pathogenic role in EAE. This question has not yet been addressed in detail in the literature. It is suspected that MOG-specific antibodies play a role in effector cell maturation and/or trafficking because the lack of antibodies is not accompanied by an impaired $T$ cell priming (similar to our observation) [140]. Furthermore, earlier demyelination and larger cellular infiltrates were detected in Th mice (high titer of endogenous MOG-specific antibodies), suggesting a role for antibodies in demyelination [143]. 


\section{Summary and Conclusion}

Beneficial B cell-depleting therapy in MS patients reveals a critical role for B cells to be involved in the pathogenesis of MS. However, the mechanisms of how B cells contribute to the autoimmune responses are still elusive. We generated a model enabling us to visualize and analyze antigen-specific $T$ and $B$ cells during the course of actively induced EAE, both in the periphery and in the CNS, their target organ. For this purpose we isolated fluorescently labeled MOG-specific $T$ cells from 2D2 mice and MOG-specific B cells from Th mice and injected them i.v. into OT-II mice followed by immunization with $M_{\mathrm{OOG}}$ 35-55. OT-II mice were resistant to EAE induction. This resistance could be overcome by the transfer of MOG-specific T cells. An additional transfer of MOG-specific $B$ cells resulted in an accelerated disease onset and an increased susceptibility for EAE. This experimental approach offers the advantage to focus on the pathogenic $T$ and $B$ cells due to their fluorescent label. Moreover, it permits the study of the direct impact of genetically modified lymphocytes on the course of EAE. We employed the active immunization model to ensure an antigen-specific in vivo activation. Intensive investigations on the impact of MOG-specific B cells on MOG-specific T cells during the course of EAE revealed that they did not play a critical role in enhancing $\mathrm{T}$ cell proliferation, activation or differentiation either in the activation phase or on the verge of the EAE onset. The accelerated disease onset in $\mathrm{T}$ and $\mathrm{B}$ cell-supplemented mice was accompanied by a higher number of CNS infiltrated $T$ cells and not by an enhanced $T$ cell reactivation, leading to the conclusion that MOG-specific B cells play a dispensable role in the reactivation step in the CNS. Moreover, MOG-specific B cells did not influence myeloid cell mobilization and adhesion molecule or chemokine expression of spinal cord microvessel endothelial cells. However, in the presence of MOG-specific B cells, peripheral MOG-specific $T$ cells slightly upregulate the chemokine receptors CCR6 and CXCR4, leading to speculations about whether B cells might influence $T$ cell trafficking. To investigate the mechanism utilized by $B$ cells to contribute to the accelerated EAE onset, we performed different clinical experiments. Because activated NP-specific B cells had no impact on the EAE onset it is clear that the accelerated onset is determined by the antigen-specificity of $B$ cells rather than by their activation status. Moreover, we detected MOG protein-specific antibodies of the IgG isotype in $T$ and $B$ cell-supplemented mice, but not in $T$ cell-supplemented mice 
before the appearance of first clinical signs. XBP-1 is a transcription factor critically involved in the development of plasma cells. Thus, we employed MOG-specific XBP1-deficient $B$ cells for adoptive transfer experiments to study the role of antibodies on the onset of EAE. XBP-1-deficient $B$ cells did not accelerate the onset of EAE as XBP-1-sufficient $B$ cells did, suggesting a disease-promoting role for anti-MOG protein specific antibodies in EAE. In conclusion, our established model provides a tool not only to track and analyze antigen-specific lymphocytes during EAE, but also to study the consequences of different lymphocyte supplementation on the course of disease. In our work, MOG-specific B cells and their utilized mechanisms did not enhance $T$ cell proliferation, activation or differentiation in the periphery, but their interaction with MOG-specific T cells seem to trigger their CNS infiltration. Although several publications suggest a dispensable role for $B$ cells in MOG peptide-induced $E A E$, our experimental data provide evidence that this may not be true: autoantigenspecific B cells can become stimulated by peptide antigens, resulting in their participation in the autoimmune response. 


\section{References}

1. Kawai, T. and S. Akira, The role of pattern-recognition receptors in innate immunity: update on Toll-like receptors. Nat Immunol. 11(5): p. 373-84.

2. Kono, H. and K.L. Rock, How dying cells alert the immune system to danger. Nat Rev Immunol, 2008. 8(4): p. 279-89.

3. Ozinsky, A., et al., The repertoire for pattern recognition of pathogens by the innate immune system is defined by cooperation between toll-like receptors. Proc Natl Acad Sci U S A, 2000. 97(25): p. 13766-71.

4. Geissmann, F., S. Jung, and D.R. Littman, Blood monocytes consist of two principal subsets with distinct migratory properties. Immunity, 2003. 19(1): p. 71-82.

5. Kurihara, T., et al., Defects in macrophage recruitment and host defense in mice lacking the CCR2 chemokine receptor. J Exp Med, 1997. 186(10): p. 1757-62.

6. Huffnagle, G.B., et al., The role of monocyte chemotactic protein-1 (MCP-1) in the recruitment of monocytes and CD4+ $T$ cells during a pulmonary Cryptococcus neoformans infection. J Immunol, 1995. 155(10): p. 4790-7.

7. $\mathrm{Qu}, \mathrm{C}$., et al., Role of CCR8 and other chemokine pathways in the migration of monocyte-derived dendritic cells to lymph nodes. J Exp Med, 2004. 200(10): p. 123141.

8. Nurieva, R.I. and Y. Chung, Understanding the development and function of $T$ follicular helper cells. Cell Mol Immunol. 7(3): p. 190-7.

9. Hsieh, C.S., et al., Development of TH1 CD4+ T cells through IL-12 produced by Listeria-induced macrophages. Science, 1993. 260(5107): p. 547-9.

10. Macatonia, S.E., et al., Dendritic cells produce $I L-12$ and direct the development of Th1 cells from naive CD4+ T cells. J Immunol, 1995. 154(10): p. 5071-9.

11. Jacobson, N.G., et al., Interleukin 12 signaling in Thelper type 1 (Th1) cells involves tyrosine phosphorylation of signal transducer and activator of transcription (Stat)3 and Stat4. J Exp Med, 1995. 181(5): p. 1755-62.

12. Afkarian, M., et al., T-bet is a STAT1-induced regulator of IL-12R expression in naive CD4+ T cells. Nat Immunol, 2002. 3(6): p. 549-57.

13. Fort, M.M., et al., IL-25 induces IL-4, IL-5, and IL-13 and Th2-associated pathologies in vivo. Immunity, 2001. 15(6): p. 985-95.

14. Quelle, F.W., et al., Cloning of murine Stat6 and human Stat6, Stat proteins that are tyrosine phosphorylated in responses to IL-4 and IL-3 but are not required for mitogenesis. Mol Cell Biol, 1995. 15(6): p. 3336-43.

15. Zheng, W. and R.A. Flavell, The transcription factor GATA-3 is necessary and sufficient for Th2 cytokine gene expression in CD4 T cells. Cell, 1997. 89(4): p. 58796.

16. Bettelli, E., et al., Reciprocal developmental pathways for the generation of pathogenic effector TH17 and regulatory T cells. Nature, 2006. 441(7090): p. 235-8.

17. Korn, T., et al., IL-21 initiates an alternative pathway to induce proinflammatory $T(H) 17$ cells. Nature, 2007. 448(7152): p. 484-7.

18. Ivanov, II, et al., The orphan nuclear receptor RORgammat directs the differentiation program of proinflammatory IL-17+ T helper cells. Cell, 2006. 126(6): p. 1121-33.

19. Stritesky, G.L., N. Yeh, and M.H. Kaplan, IL-23 promotes maintenance but not commitment to the Th17 lineage. J Immunol, 2008. 181(9): p. 5948-55.

20. Sutton, C., et al., A crucial role for interleukin (IL)-1 in the induction of IL-17-producing $T$ cells that mediate autoimmune encephalomyelitis. J Exp Med, 2006. 203(7): p. 1685-91.

21. Langrish, C.L., et al., IL-23 drives a pathogenic $T$ cell population that induces autoimmune inflammation. J Exp Med, 2005. 201(2): p. 233-40.

22. Chen, W., et al., Conversion of peripheral CD4+CD25- naive $T$ cells to $C D 4+C D 25+$ regulatory $T$ cells by TGF-beta induction of transcription factor Foxp3. J Exp Med, 2003. 198(12): p. 1875-86. 
23. Fontenot, J.D., et al., A function for interleukin 2 in Foxp3-expressing regulatory $T$ cells. Nat Immunol, 2005. 6(11): p. 1142-51.

24. Zheng, S.G., et al., IL-2 is essential for TGF-beta to convert naive CD4+CD25- cells to CD25+Foxp3+ regulatory $T$ cells and for expansion of these cells. J Immunol, 2007. 178(4): p. 2018-27.

25. Liston, A., et al., Differentiation of regulatory Foxp3+ $T$ cells in the thymic cortex. Proc Natl Acad Sci U S A, 2008. 105(33): p. 11903-8.

26. Sakaguchi, S., Regulatory $T$ cells: key controllers of immunologic self-tolerance. Cell, 2000. 101(5): p. 455-8.

27. Takahashi, T., et al., Immunologic self-tolerance maintained by CD25(+)CD4(+) regulatory $T$ cells constitutively expressing cytotoxic $T$ lymphocyte-associated antigen 4. J Exp Med, 2000. 192(2): p. 303-10.

28. Nurieva, R.I., et al., Bcl6 mediates the development of $T$ follicular helper cells. Science, 2009. 325(5943): p. 1001-5.

29. Vogelzang, A., et al., A fundamental role for interleukin-21 in the generation of $T$ follicular helper cells. Immunity, 2008. 29(1): p. 127-37.

30. Nurieva, R.I., et al., Generation of T follicular helper cells is mediated by interleukin21 but independent of $T$ helper 1, 2, or 17 cell lineages. Immunity, 2008. 29(1): p. 138-49.

31. Sospedra, M. and R. Martin, Immunology of multiple sclerosis. Annu Rev Immunol, 2005. 23: p. 683-747.

32. Kieseier, B.C. and H.P. Hartung, Current disease-modifying therapies in multiple sclerosis. Semin Neurol, 2003. 23(2): p. 133-46.

33. Mowry, E.M., et al., Vitamin D status is associated with relapse rate in pediatric-onset multiple sclerosis. Ann Neurol. 67(5): p. 618-24.

34. Munger, K.L., et al., Vitamin $D$ intake and incidence of multiple sclerosis. Neurology, 2004. 62(1): p. 60-5.

35. Roudbari, S.A., M.M. Ansar, and A. Yousefzad, Smoking as a risk factor for development of Secondary Progressive Multiple Sclerosis: A study in IRAN, Guilan. J Neurol Sci. 330(1-2): p. 52-5.

36. Wingerchuk, D.M., Smoking: effects on multiple sclerosis susceptibility and disease progression. Ther Adv Neurol Disord. 5(1): p. 13-22.

37. Kleinewietfeld, M., et al., Sodium chloride drives autoimmune disease by the induction of pathogenic TH17 cells. Nature. 496(7446): p. 518-22.

38. Archambault, A.S., et al., Cutting edge: Conditional MHC class II expression reveals a limited role for $B$ cell antigen presentation in primary and secondary CD4 $T$ cell responses. J Immunol. 191(2): p. 545-50.

39. Haahr, S. and P. Hollsberg, Multiple sclerosis is linked to Epstein-Barr virus infection. Rev Med Virol, 2006. 16(5): p. 297-310.

40. Sadovnick, A.D., et al., A population-based study of multiple sclerosis in twins: update. Ann Neurol, 1993. 33(3): p. 281-5.

41. Sawcer, S., et al., Genetic risk and a primary role for cell-mediated immune mechanisms in multiple sclerosis. Nature. 476(7359): p. 214-9.

42. Lassmann, H., W. Bruck, and C.F. Lucchinetti, The immunopathology of multiple sclerosis: an overview. Brain Pathol, 2007. 17(2): p. 210-8.

43. Lucchinetti, C., et al., Heterogeneity of multiple sclerosis lesions: implications for the pathogenesis of demyelination. Ann Neurol, 2000. 47(6): p. 707-17.

44. Zhang, J., et al., Increased frequency of interleukin 2-responsive $T$ cells specific for myelin basic protein and proteolipid protein in peripheral blood and cerebrospinal fluid of patients with multiple sclerosis. J Exp Med, 1994. 179(3): p. 973-84.

45. Bielekova, B., et al., Expansion and functional relevance of high-avidity myelinspecific CD4+ T cells in multiple sclerosis. J Immunol, 2004. 172(6): p. 3893-904.

46. Ota, K., et al., T-cell recognition of an immunodominant myelin basic protein epitope in multiple sclerosis. Nature, 1990. 346(6280): p. 183-7. 
47. Lovett-Racke, A.E., et al., Decreased dependence of myelin basic protein-reactive $T$ cells on CD28-mediated costimulation in multiple sclerosis patients. A marker of activated/memory T cells. J Clin Invest, 1998. 101(4): p. 725-30.

48. Viglietta, $\mathrm{V}$., et al., Loss of functional suppression by $C D 4+C D 25+$ regulatory $T$ cells in patients with multiple sclerosis. J Exp Med, 2004. 199(7): p. 971-9.

49. Haas, J., et al., Reduced suppressive effect of $C D 4+C D 25$ high regulatory $T$ cells on the $T$ cell immune response against myelin oligodendrocyte glycoprotein in patients with multiple sclerosis. Eur J Immunol, 2005. 35(11): p. 3343-52.

50. Magliozzi, R., et al., Meningeal B-cell follicles in secondary progressive multiple sclerosis associate with early onset of disease and severe cortical pathology. Brain, 2007. 130(Pt 4): p. 1089-104.

51. Serafini, B., et al., Detection of ectopic B-cell follicles with germinal centers in the meninges of patients with secondary progressive multiple sclerosis. Brain Pathol, 2004. 14(2): p. 164-74.

52. Link, H. and M.A. Laurenzi, Immunoglobulin class and light chain type of oligoclonal bands in CSF in multiple sclerosis determined by agarose gel electrophoresis and immunofixation. Ann Neurol, 1979. 6(2): p. 107-10.

53. Zhou, D., et al., Identification of a pathogenic antibody response to native myelin oligodendrocyte glycoprotein in multiple sclerosis. Proc Natl Acad Sci U S A, 2006. 103(50): p. 19057-62.

54. Genain, C.P., et al., Identification of autoantibodies associated with myelin damage in multiple sclerosis. Nat Med, 1999. 5(2): p. 170-5.

55. Bar-Or, A., et al., Abnormal B-cell cytokine responses a trigger of T-cell-mediated disease in MS? Ann Neurol. 67(4): p. 452-61.

56. Barr, T.A., et al., $B$ cell depletion therapy ameliorates autoimmune disease through ablation of IL-6-producing B cells. J Exp Med. 209(5): p. 1001-10.

57. Hauser, S.L., et al., B-cell depletion with rituximab in relapsing-remitting multiple sclerosis. N Engl J Med, 2008. 358(7): p. 676-88.

58. Cross, A.H., et al., Rituximab reduces $B$ cells and $T$ cells in cerebrospinal fluid of multiple sclerosis patients. J Neuroimmunol, 2006. 180(1-2): p. 63-70.

59. Marsters, S.A., et al., Interaction of the TNF homologues BLyS and APRIL with the TNF receptor homologues BCMA and TACI. Curr Biol, 2000. 10(13): p. 785-8.

60. Seyler, T.M., et al., BLyS and APRIL in rheumatoid arthritis. J Clin Invest, 2005. 115(11): p. 3083-92.

61. Kappos, L., et al., Atacicept in multiple sclerosis (ATAMS): a randomised, placebocontrolled, double-blind, phase 2 trial. Lancet Neurol. 13(4): p. 353-63.

62. Hoffmann, F. and E. Meinl, B cells in Multiple Sclerosis: Good or bad guys?: An article for 28 May 2014 - World MS Day 2014. Eur J Immunol. 44(5): p. 1247-50.

63. Duddy, M., et al., Distinct effector cytokine profiles of memory and naive human $B$ cell subsets and implication in multiple sclerosis. J Immunol, 2007. 178(10): p. 6092-9.

64. $\mathrm{t}$ Hart, B.A., et al., A new primate model for multiple sclerosis in the common marmoset. Immunol Today, 2000. 21(6): p. 290-7.

65. Massacesi, L., et al., Active and passively induced experimental autoimmune encephalomyelitis in common marmosets: a new model for multiple sclerosis. Ann Neurol, 1995. 37(4): p. 519-30.

66. O'Connor, R.A., et al., Cutting edge: Th1 cells facilitate the entry of Th17 cells to the central nervous system during experimental autoimmune encephalomyelitis. $J$ Immunol, 2008. 181(6): p. 3750-4.

67. Kroenke, M.A., et al., IL-12- and IL-23-modulated T cells induce distinct types of EAE based on histology, CNS chemokine profile, and response to cytokine inhibition. J Exp Med, 2008. 205(7): p. 1535-41.

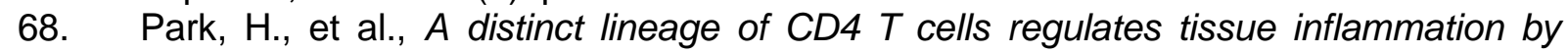
producing interleukin 17. Nat Immunol, 2005. 6(11): p. 1133-41.

69. Jager, A., et al., Th1, Th17, and Th9 effector cells induce experimental autoimmune encephalomyelitis with different pathological phenotypes. J Immunol, 2009. 183(11): p. 7169-77. 
70. Baron, J.L., et al., Surface expression of alpha 4 integrin by CD4 $T$ cells is required for their entry into brain parenchyma. J Exp Med, 1993. 177(1): p. 57-68.

71. El-Behi, M., et al., The encephalitogenicity of $T(H) 17$ cells is dependent on IL-1- and IL-23-induced production of the cytokine GM-CSF. Nat Immunol. 12(6): p. 568-75.

72. Codarri, L., et al., RORgammat drives production of the cytokine GM-CSF in helper T cells, which is essential for the effector phase of autoimmune neuroinflammation. Nat Immunol. 12(6): p. 560-7.

73. Zamvil, S.S., et al., T-cell epitope of the autoantigen myelin basic protein that induces encephalomyelitis. Nature, 1986. 324(6094): p. 258-60.

74. McRae, B.L., et al., Induction of active and adoptive relapsing experimental autoimmune encephalomyelitis (EAE) using an encephalitogenic epitope of proteolipid protein. J Neuroimmunol, 1992. 38(3): p. 229-40.

75. Mendel, I., N. Kerlero de Rosbo, and A. Ben-Nun, A myelin oligodendrocyte glycoprotein peptide induces typical chronic experimental autoimmune encephalomyelitis in $\mathrm{H}-2 \mathrm{~b}$ mice: fine specificity and $\mathrm{T}$ cell receptor $\mathrm{V}$ beta expression of encephalitogenic T cells. Eur J Immunol, 1995. 25(7): p. 1951-9.

76. Hemmer, B., J.J. Archelos, and H.P. Hartung, New concepts in the immunopathogenesis of multiple sclerosis. Nat Rev Neurosci, 2002. 3(4): p. 291-301.

77. Bettelli, E., et al., Myelin oligodendrocyte glycoprotein-specific $T$ cell receptor transgenic mice develop spontaneous autoimmune optic neuritis. J Exp Med, 2003. 197(9): p. 1073-81.

78. Bettelli, E., et al., Myelin oligodendrocyte glycoprotein-specific $T$ and $B$ cells cooperate to induce a Devic-like disease in mice. J Clin Invest, 2006. 116(9): p. 2393402.

79. Krishnamoorthy, G., et al., Spontaneous opticospinal encephalomyelitis in a doubletransgenic mouse model of autoimmune T cell/B cell cooperation. J Clin Invest, 2006. 116(9): p. 2385-92.

80. Whitham, R.H., et al., Lymphocytes from SJL/J mice immunized with spinal cord respond selectively to a peptide of proteolipid protein and transfer relapsing demyelinating experimental autoimmune encephalomyelitis. J Immunol, 1991. 146(1): p. 101-7.

81. Pollinger, B., et al., Spontaneous relapsing-remitting EAE in the SJL/J mouse: MOGreactive transgenic $T$ cells recruit endogenous MOG-specific $B$ cells. J Exp Med, 2009. 206(6): p. 1303-16.

82. Stromnes, I.M., et al., Differential regulation of central nervous system autoimmunity by $T(H) 1$ and $T(H) 17$ cells. Nat Med, 2008. 14(3): p. 337-42.

83. Muller, D.M., M.P. Pender, and J.M. Greer, A neuropathological analysis of experimental autoimmune encephalomyelitis with predominant brain stem and cerebellar involvement and differences between active and passive induction. Acta Neuropathol, 2000. 100(2): p. 174-82.

84. Hickey, W.F. and H. Kimura, Perivascular microglial cells of the CNS are bone marrow-derived and present antigen in vivo. Science, 1988. 239(4837): p. 290-2.

85. Lassmann, $\mathrm{H}$., et al., Bone marrow derived elements and resident microglia in brain inflammation. Glia, 1993. 7(1): p. 19-24.

86. Shrikant, P. and E.N. Benveniste, The central nervous system as an immunocompetent organ: role of glial cells in antigen presentation. J Immunol, 1996. 157(5): p. 1819-22.

87. Hickey, W.F., B.L. Hsu, and H. Kimura, T-lymphocyte entry into the central nervous system. J Neurosci Res, 1991. 28(2): p. 254-60.

88. Mason, D.W., et al., The fate of allogeneic and xenogeneic neuronal tissue transplanted into the third ventricle of rodents. Neuroscience, 1986. 19(3): p. 685-94.

89. Prat, A., et al., Glial cell influence on the human blood-brain barrier. Glia, 2001. 36(2): p. $145-55$.

90. Abbott, N.J., L. Ronnback, and E. Hansson, Astrocyte-endothelial interactions at the blood-brain barrier. Nat Rev Neurosci, 2006. 7(1): p. 41-53. 
91. Engelhardt, B. and R.M. Ransohoff, Capture, crawl, cross: the T cell code to breach the blood-brain barriers. Trends Immunol. 33(12): p. 579-89.

92. Osmers, I., D.C. Bullard, and S.R. Barnum, PSGL-1 is not required for development of experimental autoimmune encephalomyelitis. J Neuroimmunol, 2005. 166(1-2): p. 193-6.

93. Engelhardt, B., et al., P-selectin glycoprotein ligand 1 is not required for the development of experimental autoimmune encephalomyelitis in SJL and C57BL/6 mice. J Immunol, 2005. 175(2): p. 1267-75.

94. Borges, E., et al., P-selectin glycoprotein ligand-1 (PSGL-1) on Thelper 1 but not on $T$ helper 2 cells binds to $P$-selectin and supports migration into inflamed skin. J Exp Med, 1997. 185(3): p. 573-8.

95. Hirata, T., et al., P-Selectin glycoprotein ligand 1 (PSGL-1) is a physiological ligand for E-selectin in mediating $T$ helper 1 lymphocyte migration. J Exp Med, 2000. 192(11): p. 1669-76.

96. Yednock, T.A., et al., Prevention of experimental autoimmune encephalomyelitis by antibodies against alpha 4 beta 1 integrin. Nature, 1992. 356(6364): p. 63-6.

97. Engelhardt, B., et al., The development of experimental autoimmune encephalomyelitis in the mouse requires alpha4-integrin but not alpha4beta7-integrin. J Clin Invest, 1998. 102(12): p. 2096-105.

98. Laschinger, M. and B. Engelhardt, Interaction of alpha4-integrin with VCAM-1 is involved in adhesion of encephalitogenic $T$ cell blasts to brain endothelium but not in their transendothelial migration in vitro. J Neuroimmunol, 2000. 102(1): p. 32-43.

99. Vajkoczy, P., M. Laschinger, and B. Engelhardt, Alpha4-integrin-VCAM-1 binding mediates $G$ protein-independent capture of encephalitogenic $T$ cell blasts to CNS white matter microvessels. J Clin Invest, 2001. 108(4): p. 557-65.

100. Hutchinson, M., Natalizumab: $A$ new treatment for relapsing remitting multiple sclerosis. Ther Clin Risk Manag, 2007. 3(2): p. 259-68.

101. Johnson, K.P., Natalizumab (Tysabri) treatment for relapsing multiple sclerosis. Neurologist, 2007. 13(4): p. 182-7.

102. Piccio, L., et al., Molecular mechanisms involved in lymphocyte recruitment in inflamed brain microvessels: critical roles for P-selectin glycoprotein ligand-1 and heterotrimeric G(i)-linked receptors. J Immunol, 2002. 168(4): p. 1940-9.

103. Alt, C., M. Laschinger, and B. Engelhardt, Functional expression of the lymphoid chemokines CCL19 (ELC) and CCL 21 (SLC) at the blood-brain barrier suggests their involvement in G-protein-dependent lymphocyte recruitment into the central nervous system during experimental autoimmune encephalomyelitis. Eur J Immunol, 2002. 32(8): p. 2133-44.

104. Marlin, S.D. and T.A. Springer, Purified intercellular adhesion molecule-1 (ICAM-1) is a ligand for lymphocyte function-associated antigen 1 (LFA-1). Cell, 1987. 51(5): $\mathrm{p}$. 813-9.

105. Steiner, O., et al., Differential roles for endothelial ICAM-1, ICAM-2, and VCAM-1 in shear-resistant $T$ cell arrest, polarization, and directed crawling on blood-brain barrier endothelium. J Immunol. 185(8): p. 4846-55.

106. Laschinger, M., P. Vajkoczy, and B. Engelhardt, Encephalitogenic T cells use LFA-1 for transendothelial migration but not during capture and initial adhesion strengthening in healthy spinal cord microvessels in vivo. Eur J Immunol, 2002. 32(12): p. 3598-606.

107. Bachmann, M.F., et al., Distinct roles for LFA-1 and CD28 during activation of naive $T$ cells: adhesion versus costimulation. Immunity, 1997. 7(4): p. 549-57.

108. Wang, Y., et al., A critical role of LFA-1 in the development of Th17 cells and induction of experimental autoimmune encephalomyelytis. Biochem Biophys Res Commun, 2007. 353(4): p. 857-62.

109. Gultner, S., et al., Reduced Treg frequency in LFA-1-deficient mice allows enhanced $T$ effector differentiation and pathology in EAE. Eur J Immunol. 40(12): p. 3403-12. 
110. Archelos, J.J., et al., Inhibition of experimental autoimmune encephalomyelitis by an antibody to the intercellular adhesion molecule ICAM-1. Ann Neurol, 1993. 34(2): p. 145-54.

111. Willenborg, D.O., et al., ICAM-1-dependent pathway is not critically involved in the inflammatory process of autoimmune encephalomyelitis or in cytokine-induced inflammation of the central nervous system. J Neuroimmunol, 1993. 45(1-2): p. 14754.

112. Cayrol, R., et al., Activated leukocyte cell adhesion molecule promotes leukocyte trafficking into the central nervous system. Nat Immunol, 2008. 9(2): p. 137-45.

113. Polfliet, M.M., et al., The role of perivascular and meningeal macrophages in experimental allergic encephalomyelitis. J Neuroimmunol, 2002. 122(1-2): p. 1-8.

114. Kivisakk, P., et al., Localizing central nervous system immune surveillance: meningeal antigen-presenting cells activate $T$ cells during experimental autoimmune encephalomyelitis. Ann Neurol, 2009. 65(4): p. 457-69.

115. Kawakami, N., et al., The activation status of neuroantigen-specific $T$ cells in the target organ determines the clinical outcome of autoimmune encephalomyelitis. J Exp Med, 2004. 199(2): p. 185-97.

116. Flugel, A., et al., Migratory activity and functional changes of green fluorescent effector cells before and during experimental autoimmune encephalomyelitis. Immunity, 2001. 14(5): p. 547-60.

117. Tran, E.H., E.N. Prince, and T. Owens, IFN-gamma shapes immune invasion of the central nervous system via regulation of chemokines. J Immunol, 2000. 164(5): p. 2759-68.

118. Qin, S., et al., The chemokine receptors CXCR3 and CCR5 mark subsets of $T$ cells associated with certain inflammatory reactions. J Clin Invest, 1998. 101(4): p. 746-54.

119. Bonecchi, R., et al., Differential expression of chemokine receptors and chemotactic responsiveness of type 1 T helper cells (Th1s) and Th2s. J Exp Med, 1998. 187(1): p. 129-34.

120. Kohler, R.E., et al., Antagonism of the chemokine receptors CXCR3 and CXCR4 reduces the pathology of experimental autoimmune encephalomyelitis. Brain Pathol, 2008. 18(4): p. 504-16.

121. Sporici, R. and T.B. Issekutz, CXCR3 blockade inhibits T-cell migration into the CNS during EAE and prevents development of adoptively transferred, but not actively induced, disease. Eur J Immunol. 40(10): p. 2751-61.

122. Simpson, J., et al., Expression of the beta-chemokine receptors CCR2, CCR3 and CCR5 in multiple sclerosis central nervous system tissue. J Neuroimmunol, 2000. 108(1-2): p. 192-200.

123. Liu, L., et al., Severe disease, unaltered leukocyte migration, and reduced IFNgamma production in CXCR3-/- mice with experimental autoimmune encephalomyelitis. J Immunol, 2006. 176(7): p. 4399-409.

124. Muller, M., et al., CXCR3 signaling reduces the severity of experimental autoimmune encephalomyelitis by controlling the parenchymal distribution of effector and regulatory T cells in the central nervous system. J Immunol, 2007. 179(5): p. 2774-86.

125. Narumi, S., et al., Neutralization of IFN-inducible protein 10/CXCL10 exacerbates experimental autoimmune encephalomyelitis. Eur J Immunol, 2002. 32(6): p. 178491.

126. Fife, B.T., et al., CXCL10 (IFN-gamma-inducible protein-10) control of encephalitogenic CD4+ $T$ cell accumulation in the central nervous system during experimental autoimmune encephalomyelitis. J Immunol, 2001. 166(12): p. 7617-24.

127. Klein, R.S., et al., IFN-inducible protein 10/CXC chemokine ligand 10-independent induction of experimental autoimmune encephalomyelitis. J Immunol, 2004. 172(1): p. 550-9.

128. Tran, E.H., W.A. Kuziel, and T. Owens, Induction of experimental autoimmune encephalomyelitis in C57BL/6 mice deficient in either the chemokine macrophage inflammatory protein-1alpha or its CCR5 receptor. Eur J Immunol, 2000. 30(5): p. 1410-5. 
129. Karpus, W.J., et al., An important role for the chemokine macrophage inflammatory protein-1 alpha in the pathogenesis of the T cell-mediated autoimmune disease, experimental autoimmune encephalomyelitis. J Immunol, 1995. 155(10): p. 5003-10.

130. McCandless, E.E., et al., CXCL12 limits inflammation by localizing mononuclear infiltrates to the perivascular space during experimental autoimmune encephalomyelitis. J Immunol, 2006. 177(11): p. 8053-64.

131. Acosta-Rodriguez, E.V., et al., Surface phenotype and antigenic specificity of human interleukin 17-producing T helper memory cells. Nat Immunol, 2007. 8(6): p. 639-46.

132. Wang, C., et al., The roles of CCR6 in migration of Th17 cells and regulation of effector T-cell balance in the gut. Mucosal Immunol, 2009. 2(2): p. 173-83.

133. Esplugues, E., et al., Control of TH17 cells occurs in the small intestine. Nature. 475(7357): p. 514-8.

134. Hedrick, M.N., et al., CCR6 is required for IL-23-induced psoriasis-like inflammation in mice. J Clin Invest, 2009. 119(8): p. 2317-29.

135. Reboldi, A., et al., $\mathrm{C}-\mathrm{C}$ chemokine receptor 6-regulated entry of $\mathrm{TH}-17$ cells into the CNS through the choroid plexus is required for the initiation of EAE. Nat Immunol, 2009. 10(5): p. 514-23.

136. Keegan, M., et al., Relation between humoral pathological changes in multiple sclerosis and response to therapeutic plasma exchange. Lancet, 2005. 366(9485): p. 579-82.

137. Kitamura, D., et al., A B cell-deficient mouse by targeted disruption of the membrane exon of the immunoglobulin mu chain gene. Nature, 1991. 350(6317): p. 423-6.

138. Lyons, J.A., et al., $B$ cells are critical to induction of experimental allergic encephalomyelitis by protein but not by a short encephalitogenic peptide. Eur $\mathrm{J}$ Immunol, 1999. 29(11): p. 3432-9.

139. Molnarfi, N., et al., MHC class II-dependent B cell APC function is required for induction of CNS autoimmunity independent of myelin-specific antibodies. J Exp Med. 210(13): p. 2921-37.

140. Lyons, J.A., M.J. Ramsbottom, and A.H. Cross, Critical role of antigen-specific antibody in experimental autoimmune encephalomyelitis induced by recombinant myelin oligodendrocyte glycoprotein. Eur J Immunol, 2002. 32(7): p. 1905-13.

141. Oliver, A.R., G.M. Lyon, and N.H. Ruddle, Rat and human myelin oligodendrocyte glycoproteins induce experimental autoimmune encephalomyelitis by different mechanisms in C57BL/6 mice. J Immunol, 2003. 171(1): p. 462-8.

142. Matsushita, T., et al., Regulatory $B$ cells inhibit $E A E$ initiation in mice while other $B$ cells promote disease progression. J Clin Invest, 2008. 118(10): p. 3420-30.

143. Litzenburger, T., et al., B lymphocytes producing demyelinating autoantibodies: development and function in gene-targeted transgenic mice. J Exp Med, 1998. 188(1): p. 169-80.

144. Anderson, A.C., et al., A transgenic model of central nervous system autoimmunity mediated by CD4+ and CD8+ T and B cells. J Immunol. 188(5): p. 2084-92.

145. Johnson, K.P., et al., Agarose electrophoresis of cerebrospinal fluid in multiple sclerosis. A simplified method for demonstrating cerebrospinal fluid oligoclonal immunoglobulin bands. Neurology, 1977. 27(3): p. 273-7.

146. Schaller, M., et al., Raised levels of anti-glucose-6-phosphate isomerase IgG in serum and synovial fluid from patients with inflammatory arthritis. Ann Rheum Dis, 2005. 64(5): p. 743-9.

147. Schaller, M., D.R. Burton, and H.J. Ditzel, Autoantibodies to GPI in rheumatoid arthritis: linkage between an animal model and human disease. Nat Immunol, 2001. 2(8): p. 746-53.

148. Pihoker, C., et al., Autoantibodies in diabetes. Diabetes, 2005. 54 Suppl 2: p. S5261.

149. Winer, D.A., et al., B cells promote insulin resistance through modulation of $T$ cells and production of pathogenic IgG antibodies. Nat Med. 17(5): p. 610-7. 
150. Hjelmstrom, P., et al., B-cell-deficient mice develop experimental allergic encephalomyelitis with demyelination after myelin oligodendrocyte glycoprotein sensitization. J Immunol, 1998. 161(9): p. 4480-3.

151. Weber, M.S., et al., B-cell activation influences T-cell polarization and outcome of anti-CD20 B-cell depletion in central nervous system autoimmunity. Ann Neurol. 68(3): p. 369-83.

152. Schluesener, H.J., et al., A monoclonal antibody against a myelin oligodendrocyte glycoprotein induces relapses and demyelination in central nervous system autoimmune disease. J Immunol, 1987. 139(12): p. 4016-21.

153. Berer, K., et al., Commensal microbiota and myelin autoantigen cooperate to trigger autoimmune demyelination. Nature. 479(7374): p. 538-41.

154. Pierson, E.R., I.M. Stromnes, and J.M. Goverman, $B$ cells promote induction of experimental autoimmune encephalomyelitis by facilitating reactivation of $T$ cells in the central nervous system. J Immunol. 192(3): p. 929-39.

155. Samoilova, E.B., et al., IL-6-deficient mice are resistant to experimental autoimmune encephalomyelitis: roles of IL-6 in the activation and differentiation of autoreactive $T$ cells. J Immunol, 1998. 161(12): p. 6480-6.

156. Yanaba, K., et al., A regulatory $B$ cell subset with a unique CD1dhiCD5+ phenotype controls $T$ cell-dependent inflammatory responses. Immunity, 2008. 28(5): p. 639-50.

157. Fillatreau, S., et al., B cells regulate autoimmunity by provision of IL-10. Nat Immunol, 2002. 3(10): p. 944-50.

158. Yanaba, K., et al., The development and function of regulatory $B$ cells expressing IL10 (B10 cells) requires antigen receptor diversity and TLR signals. J Immunol, 2009. 182(12): p. 7459-72.

159. Shen, P., et al., IL-35-producing $B$ cells are critical regulators of immunity during autoimmune and infectious diseases. Nature. 507(7492): p. 366-70.

160. Yoshizaki, A., et al., Regulatory B cells control T-cell autoimmunity through IL-21dependent cognate interactions. Nature. 491(7423): p. 264-8.

161. Matsushita, T., et al., Regulatory $B$ cells (B10 cells) and regulatory $T$ cells have independent roles in controlling experimental autoimmune encephalomyelitis initiation and late-phase immunopathogenesis. J Immunol. 185(4): p. 2240-52.

162. Tran, E.H., et al., Immune invasion of the central nervous system parenchyma and experimental allergic encephalomyelitis, but not leukocyte extravasation from blood, are prevented in macrophage-depleted mice. J Immunol, 1998. 161(7): p. 3767-75.

163. Brosnan, C.F., M.B. Bornstein, and B.R. Bloom, The effects of macrophage depletion on the clinical and pathologic expression of experimental allergic encephalomyelitis. $\mathrm{J}$ Immunol, 1981. 126(2): p. 614-20.

164. Huitinga, I., et al., Suppression of experimental allergic encephalomyelitis in Lewis rats after elimination of macrophages. J Exp Med, 1990. 172(4): p. 1025-33.

165. Fife, B.T., et al., CC chemokine receptor 2 is critical for induction of experimental autoimmune encephalomyelitis. J Exp Med, 2000. 192(6): p. 899-905.

166. Huang, D.R., et al., Absence of monocyte chemoattractant protein 1 in mice leads to decreased local macrophage recruitment and antigen-specific $T$ helper cell type 1 immune response in experimental autoimmune encephalomyelitis. J Exp Med, 2001. 193(6): p. 713-26.

167. Izikson, L., et al., Resistance to experimental autoimmune encephalomyelitis in mice lacking the CC chemokine receptor (CCR)2. J Exp Med, 2000. 192(7): p. 1075-80.

168. Saederup, N., et al., Selective chemokine receptor usage by central nervous system myeloid cells in CCR2-red fluorescent protein knock-in mice. PLoS One. 5(10): p. e13693.

169. Mildner, A., et al., CCR2+Ly-6Chi monocytes are crucial for the effector phase of autoimmunity in the central nervous system. Brain, 2009. 132(Pt 9): p. 2487-500.

170. Luche, $\mathrm{H}_{\text {., }}$ et al., Faithful activation of an extra-bright red fluorescent protein in "knock-in" Cre-reporter mice ideally suited for lineage tracing studies. Eur J Immunol, 2007. 37(1): p. 43-53. 
171. Okabe, M., et al., 'Green mice' as a source of ubiquitous green cells. FEBS Lett, 1997. 407(3): p. 313-9.

172. Sonoda, E., et al., B cell development under the condition of allelic inclusion. Immunity, 1997. 6(3): p. 225-33.

173. Barnden, M.J., et al., Defective TCR expression in transgenic mice constructed using cDNA-based alpha- and beta-chain genes under the control of heterologous regulatory elements. Immunol Cell Biol, 1998. 76(1): p. 34-40.

174. Hobeika, E., et al., Testing gene function early in the $B$ cell lineage in mb1-cre mice. Proc Natl Acad Sci U S A, 2006. 103(37): p. 13789-94.

175. Hetz, C., et al., Unfolded protein response transcription factor XBP-1 does not influence prion replication or pathogenesis. Proc Natl Acad Sci U S A, 2008. 105(2): p. 757-62.

176. Madsen, L., et al., Mice lacking all conventional MHC class II genes. Proc Natl Acad Sci U S A, 1999. 96(18): p. 10338-43.

177. Wang, Y., et al., Th2 lymphoproliferative disorder of LatY136F mutant mice unfolds independently of TCR-MHC engagement and is insensitive to the action of Foxp3+ regulatory $T$ cells. J Immunol, 2008. 180(3): p. 1565-75.

178. Amor, S., et al., Identification of epitopes of myelin oligodendrocyte glycoprotein for the induction of experimental allergic encephalomyelitis in SJL and Biozzi AB/H mice. J Immunol, 1994. 153(10): p. 4349-56.

179. Kivisakk, P., et al., Expression of CCR7 in multiple sclerosis: implications for CNS immunity. Ann Neurol, 2004. 55(5): p. 627-38.

180. Mora, J.R., et al., Selective imprinting of gut-homing $T$ cells by Peyer's patch dendritic cells. Nature, 2003. 424(6944): p. 88-93.

181. King, I.L., T.L. Dickendesher, and B.M. Segal, Circulating Ly-6C+ myeloid precursors migrate to the CNS and play a pathogenic role during autoimmune demyelinating disease. Blood, 2009. 113(14): p. 3190-7.

182. Zouggari, Y., et al., $B$ lymphocytes trigger monocyte mobilization and impair heart function after acute myocardial infarction. Nat Med. 19(10): p. 1273-80.

183. Eugster, H.P., et al., IL-6-deficient mice resist myelin oligodendrocyte glycoproteininduced autoimmune encephalomyelitis. Eur J Immunol, 1998. 28(7): p. 2178-87.

184. Reimold, A.M., et al., Plasma cell differentiation requires the transcription factor XBP1. Nature, 2001. 412(6844): p. 300-7.

185. Tumanov, A., et al., Distinct role of surface lymphotoxin expressed by $B$ cells in the organization of secondary lymphoid tissues. Immunity, 2002. 17(3): p. 239-50.

186. Crowley, M.T., C.R. Reilly, and D. Lo, Influence of lymphocytes on the presence and organization of dendritic cell subsets in the spleen. J Immunol, 1999. 163(9): p. 4894900.

187. Constant, S., et al., Peptide and protein antigens require distinct antigen-presenting cell subsets for the priming of CD4+ T cells. J Immunol, 1995. 154(10): p. 4915-23.

188. Constant, S., et al., B lymphocytes can be competent antigen-presenting cells for priming CD4+ T cells to protein antigens in vivo. J Immunol, 1995. 155(8): p. 3734-41.

189. Ronchese, F. and B. Hausmann, $B$ lymphocytes in vivo fail to prime naive $T$ cells but can stimulate antigen-experienced T lymphocytes. J Exp Med, 1993. 177(3): p. 67990.

190. Crawford, A., et al., Primary $T$ cell expansion and differentiation in vivo requires antigen presentation by $B$ cells. J Immunol, 2006. 176(6): p. 3498-506.

191. Macaulay, A.E., et al., Antigen-specific $B$ cells preferentially induce CD4+ $T$ cells to produce IL-4. J Immunol, 1997. 158(9): p. 4171-9.

192. Petrovic, A., et al., LPAM (alpha 4 beta 7 integrin) is an important homing integrin on alloreactive $T$ cells in the development of intestinal graft-versus-host disease. Blood, 2004. 103(4): p. $1542-7$.

193. Berlin, C., et al., Alpha 4 beta 7 integrin mediates lymphocyte binding to the mucosal vascular addressin MAdCAM-1. Cell, 1993. 74(1): p. 185-95.

194. Zabel, B.A., et al., Human G protein-coupled receptor GPR-9-6/CC chemokine receptor 9 is selectively expressed on intestinal homing $T$ lymphocytes, mucosal 
lymphocytes, and thymocytes and is required for thymus-expressed chemokinemediated chemotaxis. J Exp Med, 1999. 190(9): p. 1241-56.

195. Austrup, F., et al., $P$ - and E-selectin mediate recruitment of T-helper-1 but not $T$ helper-2 cells into inflammed tissues. Nature, 1997. 385(6611): p. 81-3.

196. Reiss, Y., et al., CC chemokine receptor (CCR)4 and the CCR10 ligand cutaneous T cell-attracting chemokine (CTACK) in lymphocyte trafficking to inflamed skin. J Exp Med, 2001. 194(10): p. 1541-7.

197. Sigmundsdottir, $\mathrm{H}$., et al., DCs metabolize sunlight-induced vitamin D3 to 'program' $T$ cell attraction to the epidermal chemokine CCL27. Nat Immunol, 2007. 8(3): p. 28593.

198. Woska, J.R., Jr., et al., A small-molecule antagonist of LFA-1 blocks a conformational change important for LFA-1 function. J Leukoc Biol, 2001. 70(2): p. 329-34.

199. Cabanas, C. and N. Hogg, Ligand intercellular adhesion molecule 1 has a necessary role in activation of integrin lymphocyte function-associated molecule 1. Proc Natl Acad Sci U S A, 1993. 90(12): p. 5838-42.

200. Alon, R. and K. Ley, Cells on the run: shear-regulated integrin activation in leukocyte rolling and arrest on endothelial cells. Curr Opin Cell Biol, 2008. 20(5): p. 525-32.

201. Schreiner, B., et al., $P D-1$ ligands expressed on myeloid-derived APC in the CNS regulate T-cell responses in EAE. Eur J Immunol, 2008. 38(10): p. 2706-17.

202. Dogan, R.N., A. Elhofy, and W.J. Karpus, Production of CCL2 by central nervous system cells regulates development of murine experimental autoimmune encephalomyelitis through the recruitment of TNF- and iNOS-expressing macrophages and myeloid dendritic cells. J Immunol, 2008. 180(11): p. 7376-84.

203. Bailey, S.L., et al., CNS myeloid DCs presenting endogenous myelin peptides 'preferentially' polarize CD4+ $T(H)-17$ cells in relapsing EAE. Nat Immunol, 2007. 8(2): p. 172-80.

204. Deshpande, P., I.L. King, and B.M. Segal, Cutting edge: CNS CD11c+ cells from mice with encephalomyelitis polarize Th17 cells and support CD25+CD4+ $T$ cellmediated immunosuppression, suggesting dual roles in the disease process. J Immunol, 2007. 178(11): p. 6695-9.

205. Godiska, R., et al., Chemokine expression in murine experimental allergic encephalomyelitis. J Neuroimmunol, 1995. 58(2): p. 167-76.

206. Carter, S.L., et al., Induction of the genes for CxCl9 and CxCl10 is dependent on IFNgamma but shows differential cellular expression in experimental autoimmune encephalomyelitis and by astrocytes and microglia in vitro. Glia, 2007. 55(16): p. 1728-39.

207. Petit, I., et al., G-CSF induces stem cell mobilization by decreasing bone marrow SDF-1 and up-regulating CXCR4. Nat Immunol, 2002. 3(7): p. 687-94.

208. Duddy, M.E., A. Alter, and A. Bar-Or, Distinct profiles of human $B$ cell effector cytokines: a role in immune regulation? J Immunol, 2004. 172(6): p. 3422-7.

209. Harris, D.P., et al., Reciprocal regulation of polarized cytokine production by effector $B$ and $T$ cells. Nat Immunol, 2000. 1(6): p. 475-82.

210. Mitsdoerffer, M., et al., Proinflammatory $T$ helper type 17 cells are effective B-cell helpers. Proc Natl Acad Sci U S A. 107(32): p. 14292-7.

211. Hu, C.C., et al., XBP-1 regulates signal transduction, transcription factors and bone marrow colonization in B cells. Embo J, 2009. 28(11): p. 1624-36.

212. Nadine Eckert Master Thesis 2014 "The role of the co-stimulatory molecule CD28 during different phases of the Experimental Autoimmune Encephalomyelitis" 


\section{Supplementary data}
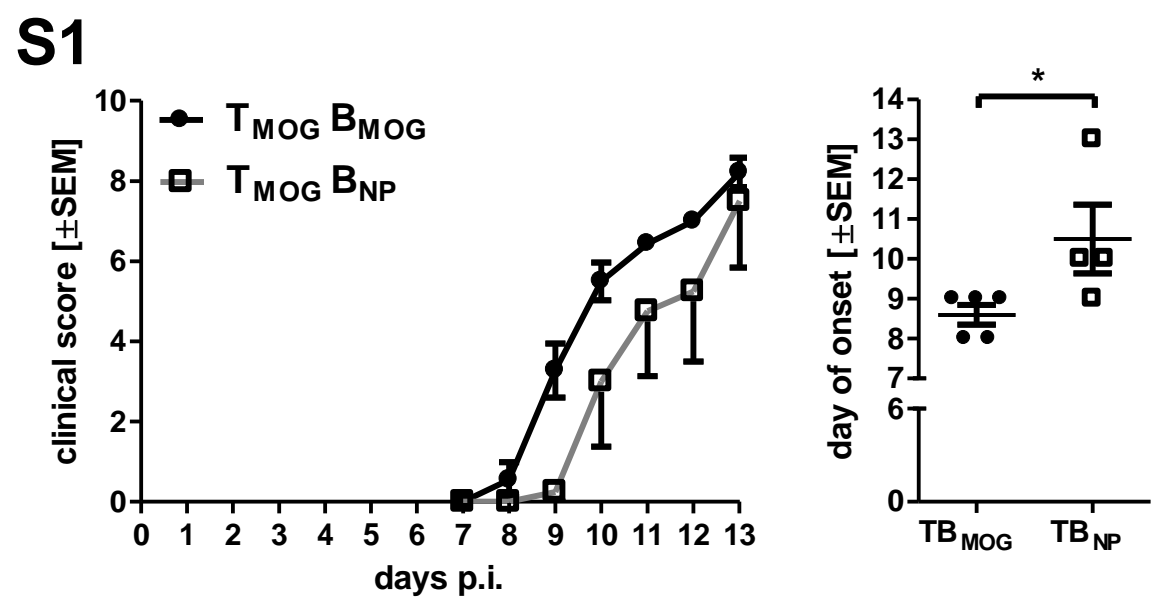

Figure S1: NP-specific $B$ cells do not accelerate the onset of EAE. MOG-specific $T$ and $B$ cells and NP-specific $B$ cells were isolated from respective donor mice and i.v. transferred $\left(3,5 \times 10^{6} \mathrm{MOG}\right.$-specific T cells+ $1 \times 10^{7} \mathrm{MOG}$-specific B cells or $3,5 \times 10^{6} \mathrm{MOG}$-specific $\mathrm{T}$ cells $+1 \times 10^{7} \mathrm{NP}$-specific B cells) into OT-II mice followed by immunization with $50 \mu \mathrm{g} \mathrm{MOG}+50 \mu \mathrm{g}$ OVA protein two days later and monitored their clinical outcome for 13 days. Data represents mean \pm SEM of 5 mice per $B_{M O G}$ group and 4 mice per $B_{N P}$ group of one experiment. Healthy animals were not included in the clinical data curve. Significant differences between means are indicated: ${ }^{*} p<0,05$, Mann-Whitney nonparametric $t$ test.


Figure S2: MOG-specific B cells do not inhibit the conversion of naïve MOG-specific $T$ cells into induced regulatory $T$ cells within the activation phase. RFP ${ }^{+}$MOG-specific FOXP3 reporter $\mathrm{T}$ cells were isolated from respective donor mice and transferred alone or together with MOG-specific B cells into OT-II mice followed by immunization with $\mathrm{MOG}_{\mathrm{p} 35-55}$ two days later. Three and four days p.i. different lymph nodes and spleen were analyzed for frequency of $\mathrm{GFP}^{+}(=$ FOXP3 ${ }^{+}$) cells among the $\mathrm{CD}^{+} \mathrm{RFP}^{+}$cells by flow cytometry. One bar represents 1 mouse per time point. Data were generated by Leon Hosang during his 8-week lab rotation. 
S3
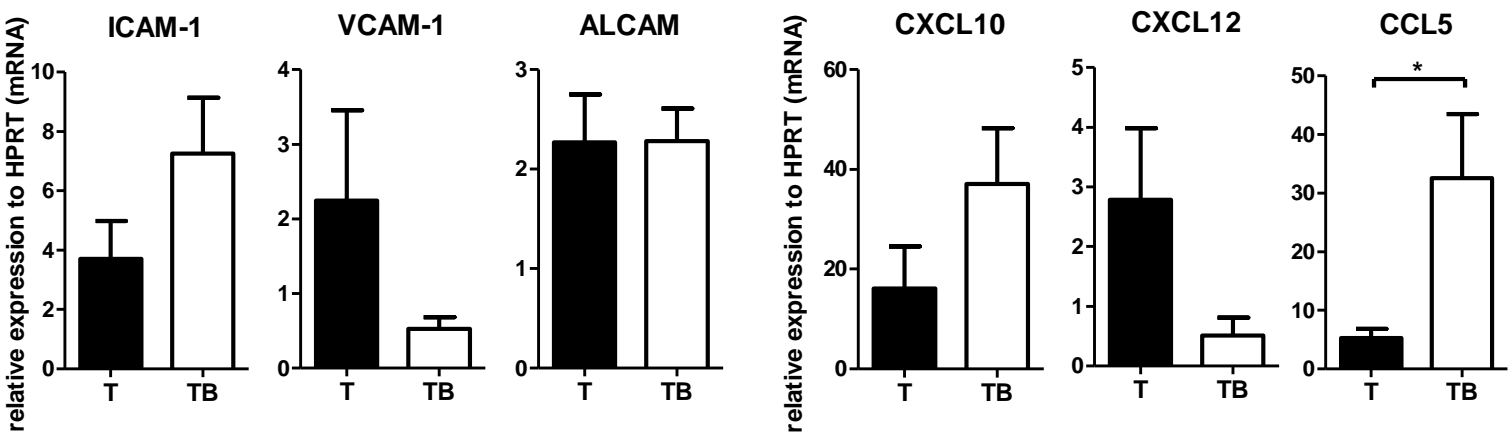

Figure S3: Spinal cord microvessel endothelial cells upregulate chemokine expression upon reactivation of initial infiltrated $\mathrm{T}$ cells. Spinal cord microvessels were isolated from $T$ cell-injected or T and B cell-injected OT-II mice between d9-d11 p.i.(without clinical score) and endothelial cells were sorted according to their CD31 expression. Adhesion molecules (ICAM-1, VCAM-1, ALCAM) and chemokine (CXCL10, CXCL12, CCL5) (mRNA) expression analysis was performed by RT-PCR. Data represent 2 independent experiments with 4 animals per group. Data are depicted as mean \pm SEM. Significant differences between means are indicated: ${ }^{*} p>0,05$, unpaired students $t$ test


Figure S4: In vitro cytokine response of co-cultured antigen-specific lymphocytes. $1 \times 10^{6}$ MOG-specific T cells were co-cultured with $2 \times 10^{6}$ MOG-specific $B$ cells with increasing $M_{0} G_{p 35}$ 55 concentrations (left) or $1 \times 10^{6}$ OVA-specific T cells were co-cultured with $2 \times 10^{6} \mathrm{NP}$-specific B cells with increasing NP-OVAL concentrations (right) for $72 \mathrm{~h}$. Supernatants were collected and analyzed for IL-10, IL-17A, TNF $\alpha$, IFN $\gamma$, IL-6, IL-4 and IL-2 with cytometric bead array. IL-10, IL-17A, IL-4 and IL-2 were not detectable. Shown is one representative experiment out of two. 


\section{Acknowledgement}

I would like to thank Prof. Flügel- for giving me the opportunity to perform my $\mathrm{PhD}$ research in his department, but also for his support and advice throughout my thesis, especially during the last six months.

Special thanks go to my supervisor Dr. Fred Lühder, who not only organized the acquisition of all used mouse strains, but also their breeding and genotyping. Furthermore, I would like to thank him for his support, his encouragement, the proofreading of my thesis and for scientific discussions.

Moreover, I would like to thank the members of my thesis committee Prof. Wienands and Prof. Simons for their time and support of my PhD project, as well as Prof. Reichardt, Prof. Hanisch and Prof. Walter for being part of my examination board.

Thank you Tanja Litke, my direct colleague of the AG Lühder, for fruitful scientific discussions, but especially for the harmonic and supportive collaboration.

Thank you Dr. Francesca Odoardi, Dr. Dmitri Lodygin and Dr. Henrike Körner for sharing their technical experience and knowledge, especially concerning RT-PCR techniques and primer design.

I got great technical support from our technical assistants during my $\mathrm{PhD}$ thesis: Thank you Adriane for helping me with isolation of countless RNAs and for teaching me several techniques. Thank you Simone for your ELISA support, but especially for teaching me the fine art of FACS cell sorting. Thank you Regine and especially Birgit for the genotyping of countless mice. In addition, I would like to thank Martina, Nancy, Brigitte and Simon for their all-around technical support. Thanks Cathy for all the bureaucratic stuff and proofreading of my thesis, Micheal Blessmann for animal care-taking and Omar for PC stuff.

Moreover, I would like to thank the other PhD students of our group (Alex, Christian, Corinna, Giuseppe and Manuel) for the nice and funny working atmosphere in the lab, but also for being a friend (day and night)! Special thanks to Michael, who not only delivered me insights into the world of myeloid cells, but also for performing the half-marathon in 1:44h! Thank you to Jan and Leon, my former 8week internship students, who worked with me on the project.

Thanks to my parents, my sisters, my Oma and my friends for their patience, support and interest in my scientific work. 
Last but not least I would like to thank the most important participants of my work: Thank you C57bl/6 and all genetic variants for giving your life for scientific research. I am awfully sorry! 


\section{Curriculum vitae}

Name

Data of Birth

Place of Birth

Contact

\author{
Anne-Christine Flach \\ 29.01.1985 \\ Remscheid, NRW \\ Institute for Multiple Sclerosis Research \\ Department of Neuroimmunology \\ Waldweg 33 \\ 37073 Göttingen \\ Phone: +49- (0)551-39-13344 \\ Email: Anne.flach@med.uni-goettingen.de
}

\section{Education}

06/2010- present

PhD- student, Institute for Multiple Sclerosis Research,

Department of Neuroimmunology, UMG Göttingen

PhD Program of the Göttingen Center for Molecular Biosciences (GZMB) "Molecular Biology of Cells"

07/2009- 05/2010 Research assistant, Gastroenterology, Infectiology and Rheumatology, Charité Berlin

06/2008- 05/2009 Diploma Thesis "Characterization and cloning of novel FOXP3dependent marker genes in regulatory T cells" Life and Medical Science Institute, Department of Genomics and Immunoregulation, Rheinische-Friedrich-Wilhelm University Bonn

10/2004- 05/2009 Diploma in biology (1.0), University of Cologne

09/1995- 06/2004 Abitur (2.0), Gertrud-Bäumer Gymnasium Remscheid

\section{Publications}

Neumann,K., Kruse,N., Szilagyi B., Erben,U., Rudolph,C., Flach,A., Zeitz,M., Hamann,A. and Klugewitz, K. (2012) Connecting liver and gut: Murine liver sinusoidal endothelium induces gut tropism of $\mathrm{CD}^{+} \mathrm{T}$ cells via retinoic acid. HEPATOLOGY 55:1976-1984

Lee,D-H., Geyer,E., Flach,A., Jung,K., Gold,R., Flügel,A., Linker,R.A., Lühder, F. (2012) Central nervous system rather than immune cell-derived BDNF mediates axonal protective effects early in autoimmune demyelination. Acta Neuropathologica, Volume 123, Issue 2, pp 247-258.

\section{Poster presentation}

2011 The Spring School of Immunology, Ettal, Germany

$20112^{\text {nd }}$ GGNB Science Day, Göttingen

$20133^{\text {rd }}$ GGNB Science Day, Göttingen 